\title{
Texture of single vanishing subtrace in neutrino mass matrix
}

\author{
A. Ismael,,$^{1,2, *}$ M. AlKhateeb, ${ }^{3,4, \dagger}$ N. Chamoun $\oplus^{5, \$}$ and E. I. Lashin ${ }^{1,2,6,8}$ \\ ${ }^{1}$ Department of Physics, Faculty of Science, Ain Shams University, Cairo 11566, Egypt \\ ${ }^{2}$ Centre for Fundamental Physics, Zewail City of Science and Technology, \\ 6 October City, Giza 12578, Egypt \\ ${ }^{3}$ Department of Physics, Faculty of Science, Damascus University, Damascus, Syria \\ ${ }^{4}$ Département de Physique, Universityé de Cergy-Pontoise, F-95302 Cergy-Pontoise, France \\ ${ }^{5}$ Physics Department, HIAST, P.O. Box 31983, Damascus, Syria \\ ${ }^{6}$ The Abdus Salam ICTP, P.O. Box 586, 34100 Trieste, Italy
}

(Received 22 June 2020; accepted 18 January 2021; published 22 February 2021)

\begin{abstract}
We consider a texture for the neutrino mass matrix characterized by one vanishing $2 \times 2$ subtrace. We analyze phenomenologically and analytically all the six possible patterns, and show that all nonsingular ones are able to accommodate the experimental bounds, whereas singular patterns allow for only four inverted-hierarchy type textures. We then present some possible realizations of this texture, within seesaw scenarios, either directly or indirectly by relating it to zero textures.
\end{abstract}

DOI: 10.1103/PhysRevD.103.035020

\section{INTRODUCTION}

The fact that neutrinos are massive was the first firm sign of physics beyond standard model [1]. Many flavor models for neutrino mass matrix were conceived, motivated by phenomenological data on neutrino oscillations [2]. Zero textures were studied extensively [3-5], but other forms of textures were equally studied, such as zero minors [6] and partial $\mu-\tau$ symmetry textures [7].

The objective of this work is to study the texture characterized by one vanishing subtrace, motivated by many factors. First, a particular texture of vanishing two subtraces was studied in [8], where analytical expressions for the measurable neutrino observables were derived, and numerical analysis was done to show that 8 patterns, out of the 15 independent ones, can accommodate data. Second, the so-called $\mu-\tau$ antisymmetry texture was studied in, say, Ref. [9]. This texture, in addition to the condition $\left(M_{e \mu}=-M_{e \tau}\right)$ which can also be implemented in $\mu-\tau$ symmetry textures [7], included also a certain vanishing subtrace condition $\left(M_{\mu \mu}+M_{\tau \tau}=0\right)$. Third, the one vanishing $2 \times 2$ subtrace texture can actually be seen as a generalization of the zero texture when the latter is regarded

\footnotetext{
*ahmedEhusien@sci.asu.edu.eg

†mohkha88@gmail.com

*nidal.chamoun@hiast.edu.sy

§slashin@zewailcity.edu.eg; elashin@ictp.it
}

Published by the American Physical Society under the terms of the Creative Commons Attribution 4.0 International license. Further distribution of this work must maintain attribution to the author(s) and the published article's title, journal citation, and DOI. Funded by SCOAP. as a vanishing $1 \times 1$ subtrace texture. Fourth, since the equal entries texture has been of interest recently, either in hybrid texture (e.g., Ref. [10]) or simply in assuming two such equalities (e.g., Ref. [11]), and since such an equality condition is closely related to a condition of the vanishing sum of the corresponding two entries, so studying textures of vanishing subtrace adds further analysis into these studies. We implement the new experimental bounds of [12], with the new updates on the nonvanishing value of the smallest mixing matrix [13], and carry out a complete numerical analysis, where all the free parameters are scanned within their experimentally accepted ranges. We discuss nonsingular patterns having all the neutrino masses nonvanishing and singular patterns where one of the masses is zero. We find all six nonsingular textures able to accommodate the experimental data. As to singular textures, only four textures can accommodate data of the inverted hierarchy type. We then address the question of how to realize such a vanishing subtrace texture. First, we relate the symmetry imposing the vanishing subtrace pattern to another symmetry which forces zeros at specific locations. The former pattern arises upon rotating the zerotexture form. Although the texture form is imposed at high scale, however, many arguments [14] were presented in favor of keeping the form when running into low scale. The method we suggest for realizing the vanishing subtrace texture applies only to four out of six possible patterns. However, it is generic enough to be applicable to any specific texture related by unitary transformation to zero textures, and we apply it successfully within type I and type II seesaw scenarios. Second, we present direct realizations of the textures within type I and type II seesaw 
scenarios, without relating them to zero textures, based on discrete symmetries.

The plan of the paper is as follows. In Sec. II, we present the notation and adopted conventions. In Sec. III, we explain the method to follow for the phenomenology. In Sec. IV, we present the analysis of all the nonsingular six patterns of one vanishing subtrace supplemented with one single table summarizing all the predictions of the various patterns. Subsections therein correspond to these different patterns where for each one we report the relevant defining quantities, correlation plots, and one representative point for each type of hierarchy. We repeat the analysis in Sec. V for the singular patterns. Section VI outlines the problem of how to build models implementing the vanishing subtrace texture whether it be directly or indirectly. In Sec. VII, we develop the generic method relating the symmetry of vanishing subtraces to that of zero textures and use it as an indirect method for getting the vanishing subtrace texture. In Sec. VIII, we clarify the notion of flavor basis which is of paramount relevance in our discussion. The aforementioned indirect method is applied within type I seesaw scenarios in order to realize invertible (singular) vanishing subtrace textures in Sec. IX (10). We reapply this indirect method in Sec. XI but within the type II seesaw scenario. In Secs. XII and XIII, we present, respectively, a direct way to impose the textures within type I and type II seesaw scenarios. The summary and conclusion are presented in Sec. 14.

\section{NOTATION}

In the "flavor" basis, where the charged lepton mass matrix is diagonal and thus the observed neutrino mixing matrix comes solely from the neutrino sector, we have

$$
\begin{aligned}
& M_{\nu}=V_{\mathrm{PMNS}}\left(\begin{array}{ccc}
m_{1} & 0 & 0 \\
0 & m_{2} & 0 \\
0 & 0 & m_{3}
\end{array}\right)\left(V_{\mathrm{PMNS}}\right)^{T}, \\
& P=\operatorname{diag}\left(e^{i \rho}, e^{i \sigma}, 1\right), \quad U=R_{23}\left(\theta_{23}\right) R_{13}\left(\theta_{13}\right) \operatorname{diag}\left(1, e^{-i \delta}, 1\right) R_{12}\left(\theta_{12}\right), \\
& V_{\mathrm{PMNS}}=U P=\left(\begin{array}{ccc}
c_{12} c_{13} e^{i \rho} & s_{12} c_{13} e^{i \sigma} & s_{13} \\
\left(-c_{12} s_{23} s_{13}-s_{12} c_{23} e^{-i \delta}\right) e^{i \rho} & \left(-s_{12} s_{23} s_{13}+c_{12} c_{23} e^{-i \delta}\right) e^{i \sigma} & s_{23} c_{13} \\
\left(-c_{12} c_{23} s_{13}+s_{12} s_{23} e^{-i \delta}\right) e^{i \rho} & \left(-s_{12} c_{23} s_{13}-c_{12} s_{23} e^{-i \delta}\right) e^{i \sigma} & c_{23} c_{13}
\end{array}\right),
\end{aligned}
$$

where $R_{i j}\left(\theta_{i j}\right)$ is the rotation matrix in the $(i, j)$ plane by angle $\theta_{i j}$ and $s_{12} \equiv \sin \theta_{12} \ldots$. Note that in this adopted parametrization, the third column of $V_{\text {PMNS }}$ is real.

The mass spectrum is classified into two categories:

(i) Normal hierarchy: characterized by $m_{1}<m_{2}<m_{3}$ and is denoted by $\mathbf{N}$.

(ii) Inverted hierarchy: characterized by $m_{3}<m_{1}<m_{2}$ and is denoted by $\mathbf{I}$.

The neutrino mass-squared differences, characterizing, respectively, solar and atmospheric neutrino masssquared differences together with their ratio $R_{\nu}$, are defined as

$$
\begin{aligned}
\delta m^{2} & \equiv m_{2}^{2}-m_{1}^{2}, \quad \Delta m^{2} \equiv\left|m_{3}^{2}-\frac{1}{2}\left(m_{1}^{2}+m_{2}^{2}\right)\right|, \\
R_{\nu} & =\delta m^{2} / \Delta m^{2} .
\end{aligned}
$$

Two parameters that put bounds on the neutrino mass scales, through studying beta-decay kinematics and neutrinoless double-beta decay, are the effective electronneutrino mass,

$$
m_{e}=\sqrt{\sum_{i=1}^{3}\left(\left|V_{e i}\right|^{2} m_{i}^{2}\right)},
$$

and the effective Majorana mass term $m_{e e}$,

$$
m_{e e}=\left|m_{1} V_{e 1}^{2}+m_{2} V_{e 2}^{2}+m_{3} V_{e 3}^{2}\right|=\left|M_{\nu 11}\right| .
$$

Cosmological observations put bounds on the "sum" parameter $\Sigma$ :

$$
\Sigma=\sum_{i=1}^{3} m_{i}
$$

The last measurable quantity we shall consider is the Jarlskog rephasing invariant defined by

$$
J=s_{12} c_{12} s_{23} c_{23} s_{13} c_{13}^{2} \sin \delta .
$$

The experimental bounds for the oscillation parameters are summarized in Table I. 
TABLE I. Allowed $1-2-3 \sigma$ ranges for the neutrino oscillation parameters: mixing angles, Dirac phase $\delta$, masssquare differences together with the $R_{\nu}$ parameter, taken from the global fit to neutrino oscillation data [12]. The quantities $\delta m^{2}, \Delta m^{2}$, and $R_{\nu}$ are, respectively, defined as $m_{2}^{2}-m_{1}^{2},\left|m_{3}^{2}-\left(m_{1}^{2}+m_{2}^{2}\right) / 2\right|$, and $\delta m^{2} / \Delta m^{2}$. Normal and inverted hierarchies are, respectively, denoted by $\mathrm{NH}$ and $\mathrm{IH}$.

\begin{tabular}{lccccc}
\hline \hline Parameter & Hierarchy & Best fit & $1 \sigma$ & $2 \sigma$ & $3 \sigma$ \\
\hline$\delta m^{2}\left(10^{-5} \mathrm{eV}^{2}\right)$ & $\mathrm{NH}, \mathrm{IH}$ & 7.37 & {$[7.21,7.54]$} & {$[7.07,7.73]$} & {$[6.93,7.96]$} \\
$\Delta m^{2}\left(10^{-3} \mathrm{eV}^{2}\right)$ & $\mathrm{NH}$ & 2.53 & {$[2.50,2.57]$} & {$[2.45,2.61]$} & {$[2.41,2.65]$} \\
& $\mathrm{IH}$ & 2.51 & {$[2.47,2.54]$} & {$[2.43,2.58]$} & {$[2.39,2.62]$} \\
$R_{\nu}$ & $\mathrm{NH}$ & 0.029 & {$[0.028,0.030]$} & {$[0.027,0.031]$} & {$[0.026,0.033]$} \\
& $\mathrm{IH}$ & 0.029 & {$[0.028,0.030]$} & {$[0.027,0.032]$} & {$[0.026,0.033]$} \\
$\theta_{12}\left({ }^{\circ}\right)$ & $\mathrm{NH}, \mathrm{IH}$ & 33.02 & {$[32.02,34.09]$} & {$[30.98,35.30]$} & {$[30.00,36.51]$} \\
$\theta_{13}\left({ }^{\circ}\right)$ & $\mathrm{NH}$ & 8.43 & {$[8.30,8.55]$} & {$[8.11,8.74]$} & $[7.92,8.90]]$ \\
& $\mathrm{IH}$ & 8.45 & {$[8.27,8.59]$} & {$[8.08,8.78]$} & $[7.92,8.94]]$ \\
$\theta_{23}\left({ }^{\circ}\right)$ & $\mathrm{NH}$ & 40.69 & {$[39.82,41.89]$} & {$[38.93,43.29]$} & $[38.10,51.66]]$ \\
& $\mathrm{IH}$ & 42.42 & {$[40.23,42.02] \cup$} & {$[39.18,44.02] \cup$} & {$[38.29,52.90]$} \\
& & & {$[48.86,51.06]$} & {$[46.89,52.01]$} & \\
$\delta\left({ }^{\circ}\right)$ & $\mathrm{NH}$ & 248.40 & {$[212.40,289.80]$} & {$[180.00,342.00]$} & {$[0.00,30.60] \cup$} \\
& & & & & {$[136.80,360]$} \\
& $\mathrm{IH}$ & 235.80 & {$[201.60,291.60]$} & {$[165.60,338.40]$} & {$[0,27.00] \cup$} \\
& & & & & {$[124.20,360]$} \\
\hline \hline
\end{tabular}

For the nonoscillation parameters $\Sigma, m_{e e}$, and $m_{e}$, we adopt the ranges reported in the recent Ref. [15] for the first two, while for $m_{e}$ we use more stringent values found in the earlier Ref. [16]:

$$
\left.\begin{array}{rl}
\Sigma & <0.7 \mathrm{eV} \\
m_{e e} & <0.3 \mathrm{eV} \\
m_{e} & <1.8 \mathrm{eV}
\end{array}\right\}
$$

\section{TEXTURE OF ONE-VANISHING SUBTRACE}

We denote by $\mathbf{C}_{i j}$ the texture where the subtrace corresponding to the $i j$ th element (i.e., the trace of the submatrix obtained by deleting the $i$ th row and the $j$ th column of $M_{\nu}$ ) is equal to zero. We have six possibilities of having one subtrace vanishing. Let the diagonal elements of the trace-free submatrix corresponding to $\mathbf{C}_{i j}$ be the elements at the $(a, b)$ and $(c, d)$ entries of $M_{\nu}$, then the vanishing subtrace condition is written as

$$
M_{\nu a b}+M_{\nu c d}=0
$$

and then we have

$$
\sum_{\ell=1}^{3}\left(U_{a \ell} U_{b \ell}+U_{c \ell} U_{d \ell}\right) \lambda_{l}=0
$$

with $\lambda_{1}=m_{1} e^{2 i \rho}, \lambda_{2}=m_{2} e^{2 i \sigma}$, and $\lambda_{3}=m_{3}$. This leads to

$$
\left.\begin{array}{l}
\frac{m_{1}}{m_{3}}=\frac{\operatorname{Re}\left(A_{3}\right) \operatorname{Im}\left(A_{2} e^{2 i \sigma}\right)-\operatorname{Re}\left(A_{2} e^{2 i \sigma}\right) \operatorname{Im}\left(A_{3}\right)}{\operatorname{Im}\left(A_{1} e^{2 i \rho}\right) \operatorname{Re}\left(A_{2} e^{2 i \sigma}\right)-\operatorname{Re}\left(A_{1} e^{2 i \rho}\right) \operatorname{Im}\left(A_{2} e^{2 i \sigma}\right)} \\
\frac{m_{2}}{m_{3}}=\frac{\operatorname{Im}\left(A_{3}\right) \operatorname{Re}\left(A_{1} e^{2 i \rho}\right)-\operatorname{Re}\left(A_{3}\right) \operatorname{Im}\left(A_{1} e^{2 i \rho}\right)}{\operatorname{Im}\left(A_{1} e^{2 i \rho}\right) \operatorname{Re}\left(A_{2} e^{2 i \sigma}\right)-\operatorname{Re}\left(A_{1} e^{2 i \rho}\right) \operatorname{Im}\left(A_{2} e^{2 i \sigma}\right)}
\end{array}\right\},
$$

where $A_{\alpha}$ is defined as

$$
A_{\alpha}=\left(U_{a \alpha} U_{b \alpha}+U_{c \alpha} U_{d \alpha}\right), \quad \alpha=1,2,3 .
$$

We see that knowing the mixing and phase angles we can get mass ratios. Considering now

$m_{3}=\sqrt{\frac{\delta m^{2}}{\left(\frac{m_{2}}{m_{3}}\right)^{2}-\left(\frac{m_{1}}{m_{3}}\right)^{2}}}, \quad m_{1}=m_{3} \times \frac{m_{1}}{m_{3}}, \quad m_{2}=m_{3} \times \frac{m_{2}}{m_{3}}$,

we see that knowing $\delta m^{2}$ will allow us now to compute the mass spectrum and all the neutrino observables. Thus our input parameters will be the seven parameters (three mixing angles + three phase angles + solar mass squared difference) which for the texture imposing one complex condition (two real conditions) allows us to determine the 9 degrees of freedom of the neutrino mass matrix. We then can compute all the observable quantities, test the experimental bounds in Table I of $\Delta m^{2}$ and in Eqs. (8) of the remaining 
mass bounds, and draw correlation plots of the accepted points.

Also, one should investigate the possibility, for each pattern, to have a singular (noninvertible) mass matrix. The viable singular mass matrix is characterized by one of the two masses ( $m_{1}$ for the $\mathrm{N}$ hierarchy, and $m_{3}$ for the I hierarchy) being equal to zero, as compatibility with the data prevents the simultaneous vanishing of two masses:

(i) The vanishing of $m_{1}$ together with Eqs. (3), (10), and (12) imply that the mass spectrum of $m_{2}$ and $m_{3}$ takes the values $\sqrt{\delta m^{2}}$ and $\sqrt{\Delta m^{2}+\delta m^{2} / 2}$, respectively, and we get

$$
\left.\begin{array}{l}
\Delta m^{2}=\delta m^{2}\left(\left|\frac{A_{2}}{A_{3}}\right|^{2}-\frac{1}{2}\right), \\
e^{2 i \sigma}=-\frac{A_{3} m_{3}}{A_{2} m_{2}} .
\end{array}\right\}
$$

(ii) The vanishing of $m_{3}$ together with Eqs. (3), (10), and (12) imply that the mass spectrum of $m_{2}$ and $m_{1}$ takes the values $\sqrt{\Delta m^{2}+\delta m^{2} / 2}$ and $\sqrt{\Delta m^{2}-\delta m^{2} / 2}$, respectively, and we get

$$
\left.\begin{array}{l}
\Delta m^{2}=\frac{1}{2} \delta m^{2}\left(\frac{\left|\frac{A_{1}}{A_{2}}\right|^{2}+1}{\left|\frac{A}{A_{2}}\right|^{2}-1}\right), \\
e^{2 i(\rho-\sigma)}=-\frac{A_{2} m_{2}}{A_{1} m_{1}} .
\end{array}\right\}
$$

\section{PHENOMENOLOGICAL ANALYSIS FOR NONSINGULAR TEXTURES}

The parameter space is seven-dimensional representing the parameters $\left(\theta_{12}, \theta_{13}, \theta_{23}, \delta, \rho, \sigma, \delta m^{2}\right)$ within their allowed experimental ranges, where we throw $N$ points uniformly in the corresponding parameter space and test using Eqs. (11) and (13) first to check the hierarchy type, and then to see whether the bounds of $\Delta m^{2}$ with those of Eq. (8) are satisfied. Since the experimental bounds stated in Table I are not identical for the two types of hierarchy, then the parameter spaces in both cases are different, and one is obliged to repeat the sampling in the two cases, imposing the desired type of hierarchy with the other experimental bounds on the accepted points. The number of points $N$ needed for a statistically significant sampling is found to be at least of the order $10^{7}-10^{10}$.

In each of the following subsections, labeled by the textures $\mathbf{C}_{i j}$, and for each corresponding pattern we provide the analytic expressions of the quantities $A_{\alpha}$, defined in Eq. (12), which characterize the pattern. We find that all the textures accommodate data for all types of hierarchy and at all statistical levels. All various predictions concerning the ranges spanned by mixing angles, phase angles, neutrino masses, $m_{e}, m_{e e}$, and $J$ are summarized in Table II. No signature is apparent in the case of normal ordering for the spanned ranges of neutrino masses presented in Table II. However, in the case of inverted ordering of the neutrino masses, we see that $m_{3}$ can reach a vanishing value for the textures $\mathbf{C}_{12}, \mathbf{C}_{13}$ at all $\sigma$ levels, and only at 2-3- $\sigma$-levels for the textures $\mathbf{C}_{22}$ and $\mathbf{C}_{33}$. In contrast, $m_{3}$ is never vanishing for the textures $\mathbf{C}_{11}$ and $\mathbf{C}_{23}$. Thus, the textures $\mathbf{C}_{12}, \mathbf{C}_{13}, \mathbf{C}_{22}$, and $\mathbf{C}_{33}$ are predicted to allow for singular mass matrix, as will be shown later to be the case. The ranges spanned by the parameter $J$, in Table II, show that $J$ at the $1-2-\sigma$-levels for normal ordering and $1-\sigma$-level for inverted ordering is negative in all textures, which puts the Dirac phase $\delta$ in the third and fourth quarters. Also from Table II, the ranges spanned by the phase angles $(\rho, \sigma)$ indicate that for the texture $\mathbf{C}_{12}$ in the case of normal hierarchy and at the $2 \sigma$ level there are gaps $\left(\sigma \notin\left[90^{\circ}, 150^{\circ}\right]\right.$ and $\left.\rho \notin\left[34^{0}, 101^{0}\right]\right)$, and a similar gap $\left(\rho \notin\left[0^{0}, 18^{0}\right]\right)$ for the texture $\mathbf{C}_{13}$ which becomes $\left(\rho \notin\left[0^{0}, 5^{0}\right]\right)$ at the $3 \sigma$ level. However, in the case of inverted ordering, we see at all levels that the phase $\rho$ for the texture $\mathbf{C}_{23}$ is bound to be in the interval $\left(\left[60^{0}, 120^{\circ}\right]\right)$.

We present for each texture with either hierarchy type the neutrino mass matrix obtained at one representative point chosen from the points accepted out of those generated randomly in the corresponding parameter space at the $3-\sigma$ level. The choice of the representative point is made in such a way to be as close as possible to the best fit values for mixing and Dirac phase angles.

Finally, we plot all the possible correlations at the $2-\sigma$ level. We show for each texture with either ordering 20 correlations. All correlations for each texture are organized in a single figure divided into left and right panels. The left panel of the figure consists of two columns where the first (second) column is devoted for a normal (inverted) hierarchy and shows three correlations amidst the mixing angles, three correlations amidst the phase angles, and three correlations of $\delta$ with [ $J, m_{e e}$, lowest neutrino mass (LNM)] and finally the correlation $\left(m_{3}, m_{23} \equiv \frac{m_{2}}{m_{3}}\right)$. On the other hand, we follow for the right panel of the figure the same division strategy as in the left one, but each column includes all the nine intercorrelations between the phase angles and the mixing angles, and the correlation $\left(m_{3}, m_{21} \equiv \frac{m_{2}}{m_{1}}\right)$. For the sake of convenience and easy referencing, each subfigure is labeled by three letters which indicate the vertical positioning $(a, b, c, \ldots)$, the type of ordering $(N=$ normal, $I=$ inverted $)$ and the paneling ( $L=$ left, $R=$ right). The last row in the figure thus gives information on the severity of the mass hierarchy.

Irrespective of the ordering (normal or inverted), we find in all the textures a sinusoidal correlation between $(\delta, J)$ which is a direct consequence of Eq. (7) where $J$ depends on mixing angles and Dirac phase $\delta$. The variations due to 


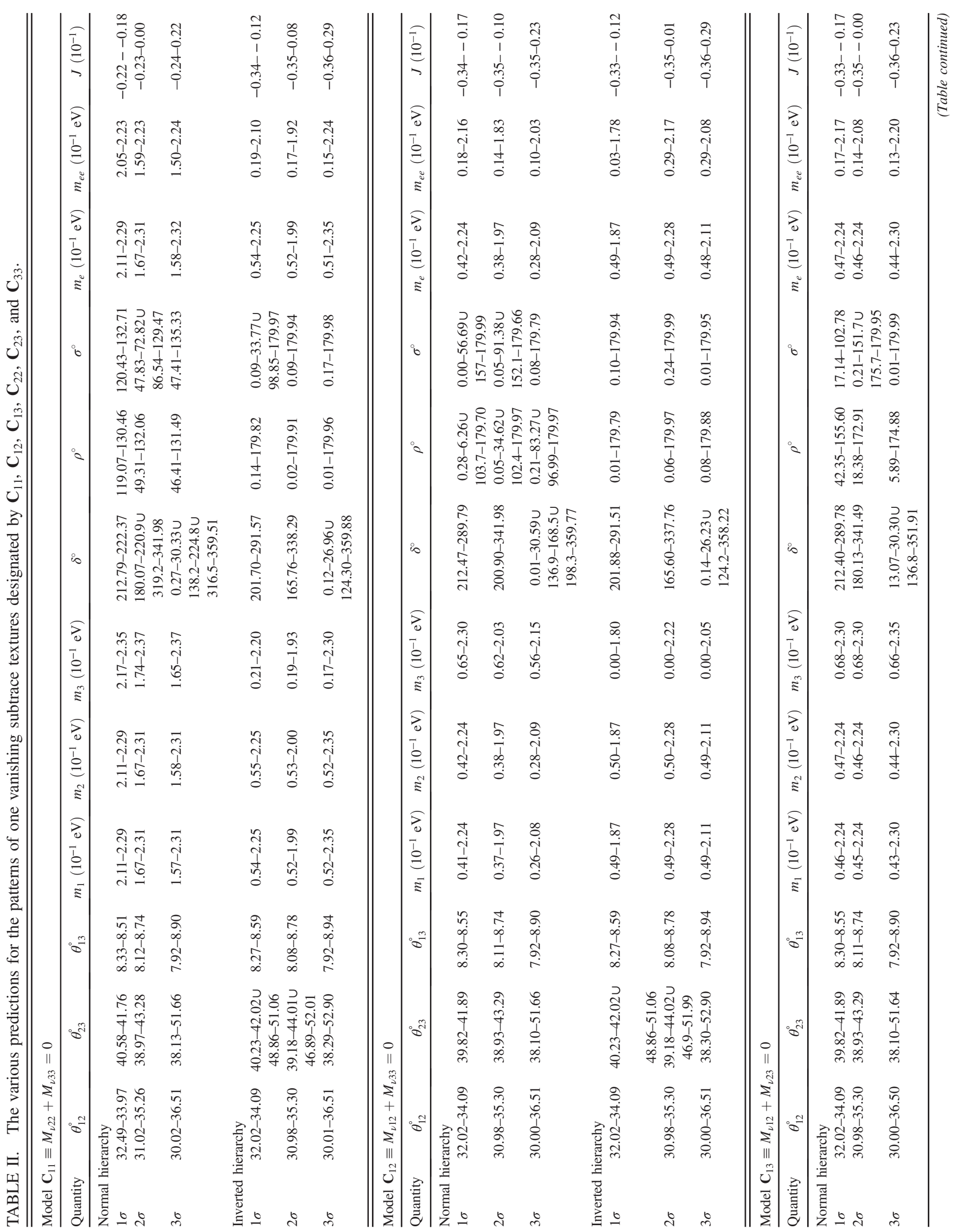




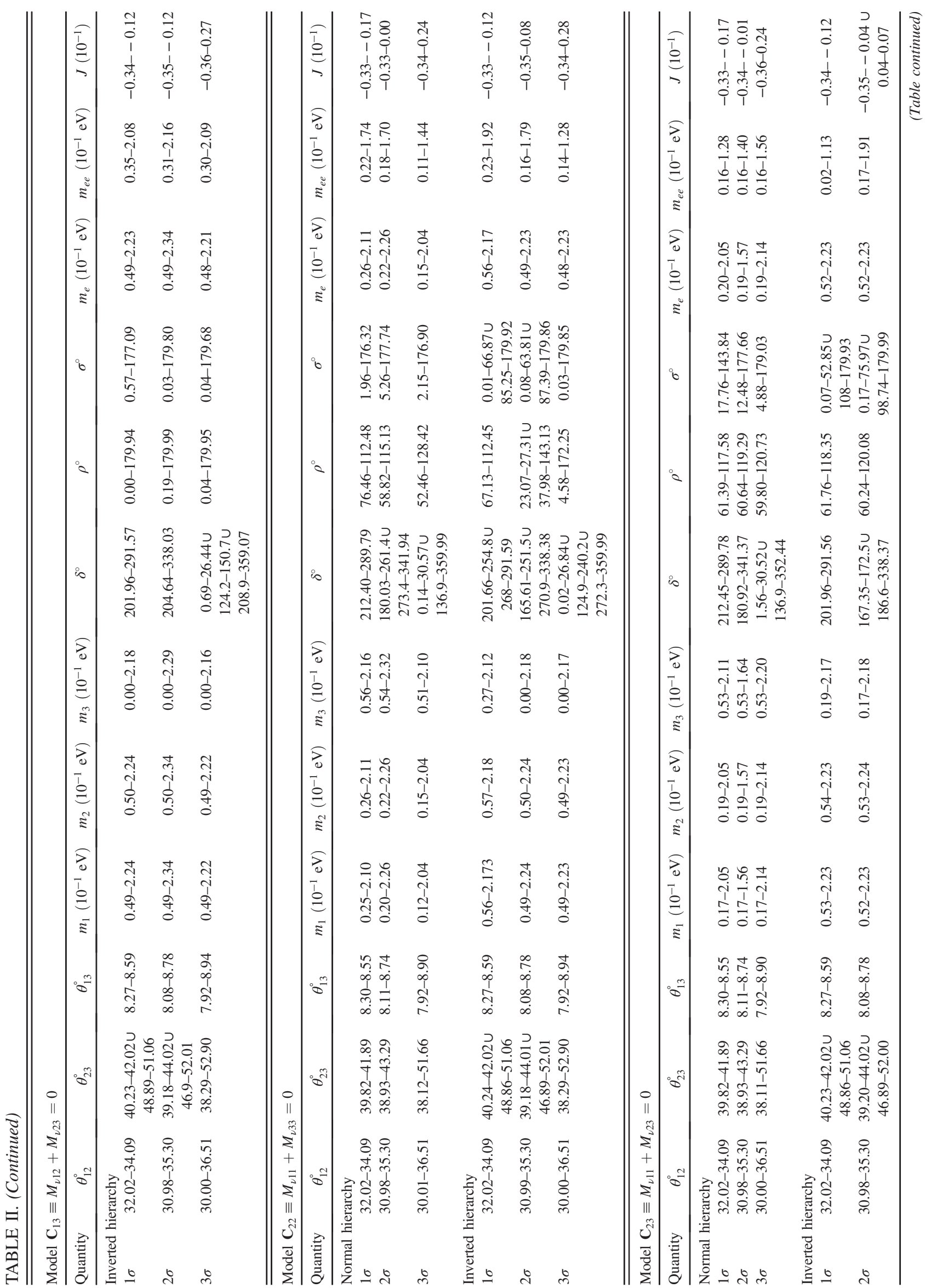


the mixing angles in this relation are tiny because of the tight range allowed for the mixing angles, and thus $J \propto \sin \delta$. The appearing sinusoidal curve is not a full sine curve which would have covered a complete cycle; rather it is a portion depending on the admissible range for $\delta$. Another generic feature that we find is the quasidegeneracy of the first two neutrino masses characterized by $m_{1} \approx m_{2}$.

In the case of normal ordering, we see, for the textures $\mathbf{C}_{12}$ and $\mathbf{C}_{13}$, sizable forbidden bands for both $\sigma$ and $\rho$ that tend to diminish as the statistical level increases, and a quasidegenerate spectrum for all neutrino masses with $\left(0.7 \leq m_{23}<1\right)$. As to the textures $\mathbf{C}_{11}$ and $\mathbf{C}_{22}$, we see that there remain persistent forbidden bands for $(\sigma, \rho)$ at all statistical levels, and that we can have a mild or moderate mass hierarchy characterized by $\left(0.4 \leq m_{23}<1\right)$ in texture $\mathbf{C}_{22}$, whereas we have a quasidegenerate spectrum for all masses with $m_{23} \approx 1$ in texture $\mathbf{C}_{11}$. Moreover, for the latter texture $\mathbf{C}_{11}$, we find two ribbons for the correlation $(\delta, \sigma)$. Regarding the texture $\mathbf{C}_{23}$, we see that there are forbidden bands for $\rho$, and that the mass hierarchy can be mild or moderate $\left(0.4 \leq m_{23} \leq 0.9\right)$. This situation repeats itself for the texture $C_{33}$ where we have $\left(0.35 \leq m_{23} \leq 0.9\right)$.

In the case of inverted ordering, we find for the texture $\mathbf{C}_{11}$ two ribbons for the correlation $(\delta, \sigma)$ and a mild or moderate mass hierarchy characterized by $\left(1<m_{23} \leq 3\right)$. For the textures $\mathbf{C}_{12}, \mathbf{C}_{13}$, and $\mathbf{C}_{22}$, we may get an acute hierarchy reaching a strength $m_{23} \approx 10^{4}$ for $\mathbf{C}_{12}$ and $m_{23} \approx$ $10^{3}$ for both $\mathbf{C}_{13}$ and $\mathbf{C}_{22}$. We get for the mass spectrum a mild hierarchy characterized by $\left(1.2 \leq m_{23} \leq 3\right)$ in the texture $\left(\mathbf{C}_{23}\right)$, where, in addition, we find forbidden bands for $(\sigma, \rho)$. Finally for the texture $\mathbf{C}_{33}$, we have again forbidden bands for $(\sigma, \rho)$, but the hierarchy can be severe reaching a strength $m_{23} \approx 10^{4}$.

\section{A. Pattern $\mathrm{C}_{11}$ : Vanishing of $M_{\nu 22}+M_{\nu 33}$}

The relevant expressions for $A_{1}, A_{2}$, and $A_{3}$, as defined in Eq. (12) for this pattern, are

$$
\begin{aligned}
& A_{1}=\left(c_{12} s_{23} s_{13}+s_{12} c_{23} e^{-i \delta}\right)^{2}+\left(c_{12} c_{23} s_{13}-s_{12} s_{23} e^{-i \delta}\right)^{2}, \\
& A_{2}=\left(s_{12} s_{23} s_{13}-c_{12} c_{23} e^{-i \delta}\right)^{2}+\left(s_{12} c_{23} s_{13}+c_{12} s_{23} e^{-i \delta}\right)^{2}, \\
& A_{3}=c_{13}^{2} .
\end{aligned}
$$

For a representative point with normal ordering, we take $\theta_{12}=33.2327^{\circ}, \theta_{23}=41.7746^{\circ}, \theta_{13}=8.5625^{\circ}, \delta=$ $189.5139^{\circ}, \rho=91.0971^{\circ}, \sigma=102.9376^{\circ}, m_{1}=0.2162 \mathrm{eV}$, $m_{2}=0.21642 \mathrm{eV}, m_{3}=0.22212 \mathrm{eV}, m_{e}=0.21642 \mathrm{eV}$, and $m_{e e}=0.20292 \mathrm{eV}$ with the corresponding mass matrix (in $\mathrm{eV}$ ): 


$$
M_{\nu}=\left(\begin{array}{ccc}
-0.2001-0.0334 i & 0.0400+0.0333 i & 0.0495-0.0230 i \\
0.0400+0.0333 i & -0.0234-0.0059 i & 0.2112-0.0014 i \\
0.0495-0.0230 i & 0.2112-0.0014 i & 0.0234+0.0059 i
\end{array}\right) .
$$

For an inverted hierarchy representative point we take $\theta_{12}=33.8335^{\circ}, \theta_{23}=42.3044^{\circ}, \theta_{13}=8.7834^{\circ}, \delta=246.8983^{\circ}$, $\rho=51.0968^{\circ}, \sigma=150.5728^{\circ}, m_{1}=0.0553 \mathrm{eV}, m_{2}=0.0560 \mathrm{eV}, m_{3}=0.0236 \mathrm{eV}, m_{e}=0.0550 \mathrm{eV}$, and $m_{e e}=$ $0.0220 \mathrm{eV}$ with the corresponding mass matrix (in $\mathrm{eV}$ ):

$$
M_{\nu}=\left(\begin{array}{ccc}
0.0014+0.0219 i & 0.0286+0.0239 i & -0.0214-0.0263 i \\
0.0286+0.0239 i & -0.0076-0.0055 i & 0.0225+0.0000 i \\
-0.0214-0.0263 i & 0.0225+0.0000 i & 0.0076+0.0055 i
\end{array}\right) .
$$

We see, from Table II, that $m_{3}$ does not approach a vanishing value in inverted type which indicates that no corresponding singular matrix exists. We see also that $J$ at $1-2 \sigma$ levels for normal ordering and $1 \sigma$ for inverted ordering is negative so the corresponding $\delta$ is at third or fourth quarters. For normal ordering, the allowed ranges for $\rho$ and $\sigma$ tend to increase as the statistical level increases reaching $\left[46.41^{\circ}, 131.49^{\circ}\right]\left(\left[47.41^{\circ}, 135.33^{\circ}\right]\right)$ for $\rho(\sigma)$ at the $3 \sigma$ level.

For the plots of Fig. 1, two ribbons for the correlation $(\delta, \sigma)$ exist for both types of hierarchy. The mass spectrum has a moderate mass hierarchy characterized by $(1<$ $\left.m_{23} \leq 3\right)$ in the inverted ordering. In contrast, the mass spectrum is quasidegenerate in the case of normal ordering where $m_{1} \approx m_{2} \approx m_{3}$.

$$
M_{\nu}=\left(\begin{array}{ccc}
0.0102-0.0146 i & -0.0255+0.0052 i & 0.0343-0.0017 i \\
-0.0255+0.0052 i & 0.0288-0.0081 i & 0.0404+0.0062 i \\
0.0343-0.0017 i & 0.0404+0.0062 i & 0.0255-0.0052 i
\end{array}\right) .
$$

For an inverted hierarchy representative point we take $\theta_{12}=33.2436^{\circ}, \theta_{23}=41.4914^{\circ}, \theta_{13}=8.6242^{\circ}, \delta=236.7486^{\circ}$, $\rho=149.9638^{\circ}, \sigma=123.9278^{\circ}, m_{1}=0.0507 \mathrm{eV}, m_{2}=0.0515 \mathrm{eV}, m_{3}=0.0115 \mathrm{eV}, m_{e}=0.0504 \mathrm{eV}$, and $m_{e e}=$ $0.0456 \mathrm{eV}$ with the corresponding mass matrix (in $\mathrm{eV}$ ):

$$
M_{\nu}=\left(\begin{array}{ccc}
0.0118-0.0440 i & 0.0093-0.0076 i & -0.0083+0.0156 i \\
0.0093-0.0076 i & -0.0194+0.0154 i & 0.0255-0.0121 i \\
-0.0083+0.0156 i & 0.0255-0.0121 i & -0.0093+0.0076 i
\end{array}\right)
$$

We see, from Table II, that $m_{3}$ can reach zero in inverted type, so we expect a possible singular texture existing. Again $J$ at $1-2 \sigma$ levels for normal ordering and $1 \sigma$ for inverted ordering is negative so the corresponding $\delta$ is at third or fourth quarters. For normal ordering, at $1-\sigma$ level there is a gap $\left[6^{\circ}, 103^{\circ}\right]\left(\left[56^{\circ}, 157^{\circ}\right]\right)$ for $\rho(\sigma)$ which becomes at $2-\sigma$ level $\left[34^{\circ}, 102^{\circ}\right]\left(\left[91^{\circ}, 152^{\circ}\right]\right)$.

For the plots of Fig. 2 in normal ordering, we find large forbidden gaps for $\rho$ and $\sigma$ and a quasidegenerate mass spectrum where $\left(0.6 \leq m_{23} \leq 0.95\right)$. As to the plots of Fig. 2 in inverted type, we see that we may get an acute hierarchy with $m_{23}$ reaching up to $10^{4}$ which reveals the possibility of vanishing $m_{3}$.

\section{Pattern $\mathrm{C}_{13}$ : Vanishing of $M_{\nu 21}+M_{\nu 23}$}

The relevant expressions for $A_{1}, A_{2}$, and $A_{3}$, as defined in Eq. (12) for this pattern, are 

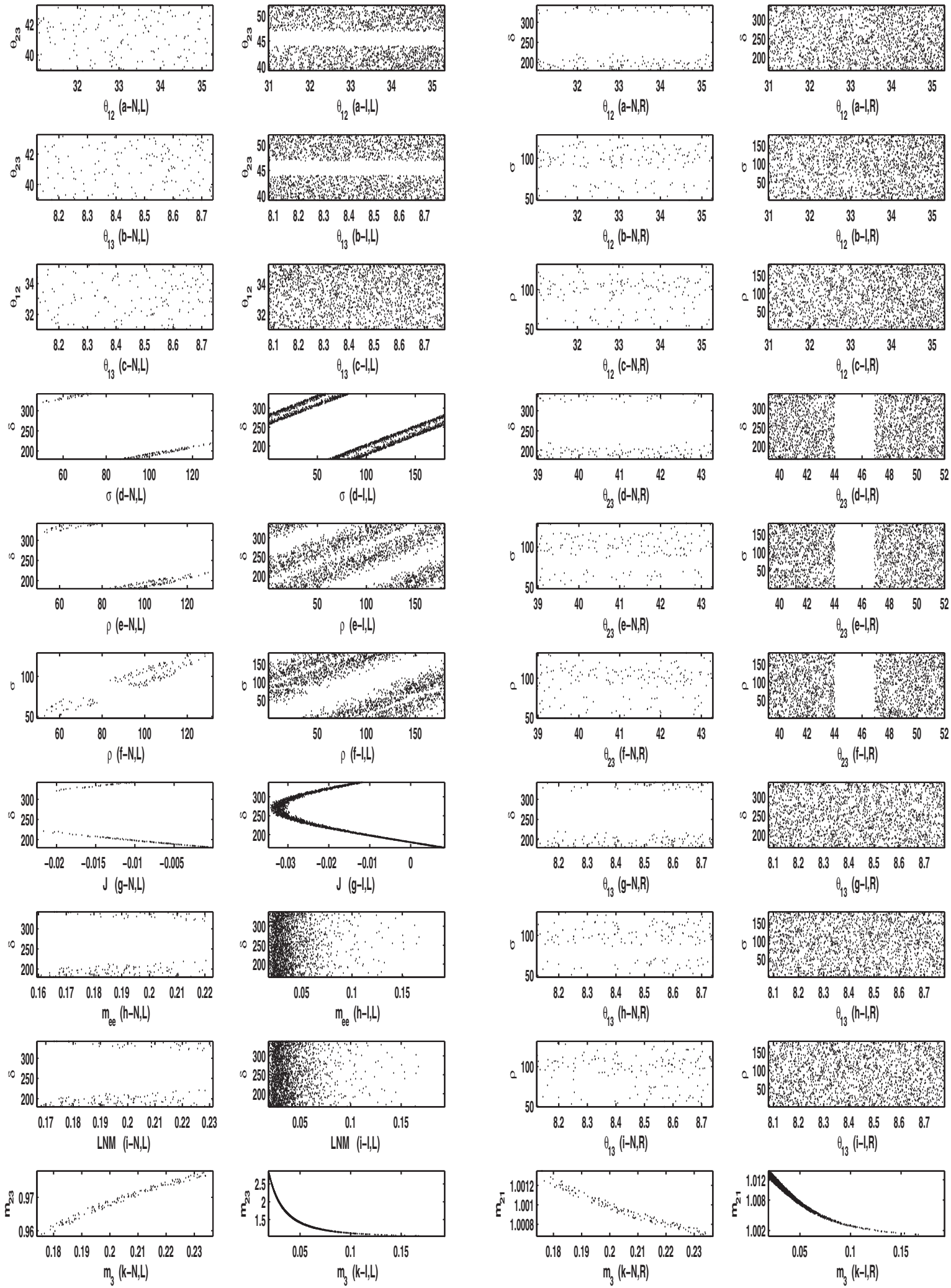

FIG. 1. Pattern $\mathbf{C}_{11} \equiv \mathbf{M}_{222}+\mathbf{M}_{\nu 33}=0$ for nonsingular mass matrices: The left panel (the left two columns) shows three correlations amidst the mixing angles, three correlations amidst the phase angles, and three correlations of $\delta$ with $J, m_{e e}$, lowest neutrino mass (LNM), and finally the correlation $\left(m_{3}, m_{23} \equiv \frac{m_{2}}{m_{3}}\right)$ for normal (N) and inverted (I) hierarchies. The right panel (the right two columns) shows all the nine intercorrelations between phase angles and mixing angles, and the correlation $\left(m_{3}, m_{21} \equiv \frac{m_{2}}{m_{1}}\right)$ for normal $(\mathrm{N})$ and inverted (I) hierarchies. Angles (masses) are evaluated in degrees (eV). 

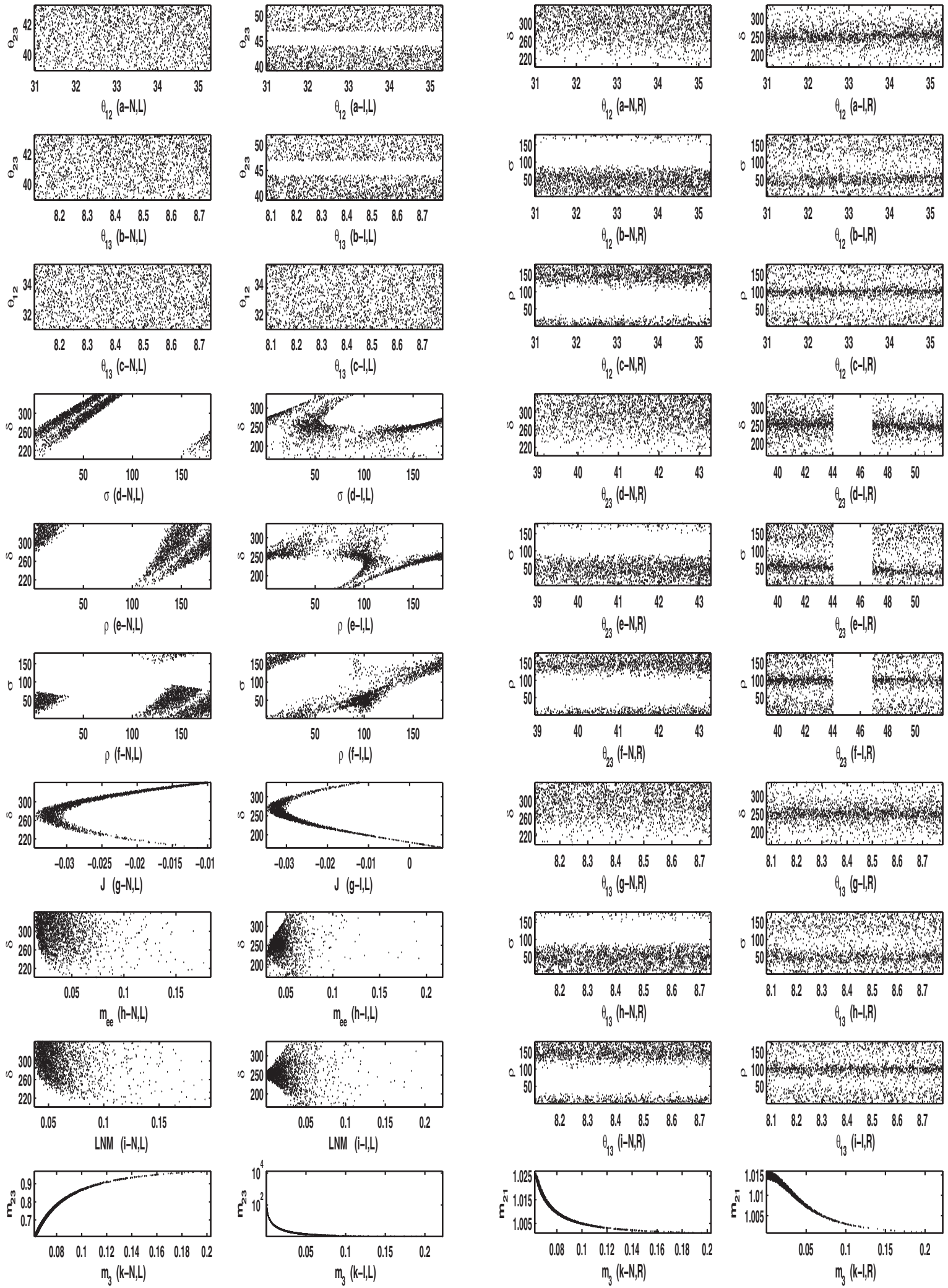

FIG. 2. Pattern $\mathbf{C}_{12} \equiv \mathbf{M}_{\nu 21}+\mathbf{M}_{\nu 33}=0$ for nonsingular mass matrices: The left panel (the left two columns) shows three correlations amidst the mixing angles, three correlations amidst the phase angles, and three correlations of $\delta$ with $J, m_{e e}$, LNM, and finally the correlation $\left(m_{3}, m_{23} \equiv \frac{m_{2}}{m_{3}}\right)$ for normal (N) and inverted (I) hierarchies. The right panel (the right two columns) shows all the nine intercorrelations between phase angles and mixing angles, and the correlation $\left(m_{3}, m_{21} \equiv \frac{m_{2}}{m_{1}}\right)$ for normal (N) and inverted (I) hierarchies. Angles (masses) are evaluated in degrees (eV). 


$$
\begin{aligned}
& A_{1}=\left(c_{12} s_{23} s_{13}+s_{12} c_{23} e^{-i \delta}\right)\left(c_{12} c_{23} s_{13}-c_{12} c_{13}-s_{12} s_{23} e^{-i \delta}\right) \\
& A_{2}=\left(s_{12} s_{23} s_{13}-c_{12} c_{23} e^{-i \delta}\right)\left(s_{12} c_{23} s_{13}-s_{12} c_{13}+c_{12} s_{23} e^{-i \delta}\right) \\
& A_{3}=c_{13} s_{23}\left(c_{23} c_{13}+s_{13}\right)
\end{aligned}
$$

For a representative point with normal ordering, we take $\theta_{12}=33.8222^{\circ}, \theta_{23}=40.4289^{\circ}, \theta_{13}=8.7721^{\circ}$, $\delta=243.7429^{\circ}, \rho=148.0834^{\circ}, \sigma=34.0333^{\circ}, m_{1}=0.1821 \mathrm{eV}, m_{2}=0.1823 \mathrm{eV}, m_{3}=0.1888 \mathrm{eV}, m_{e}=0.1823 \mathrm{eV}$, and $m_{e e}=0.0987 \mathrm{eV}$ with the corresponding mass matrix (in $\mathrm{eV}$ ):

$$
M_{\nu}=\left(\begin{array}{ccc}
0.0791-0.0590 i & -0.0909-0.0491 i & 0.0997+0.0538 i \\
-0.0909-0.0491 i & 0.1037-0.0459 i & 0.0909+0.0491 i \\
0.0997+0.0538 i & 0.0909+0.0491 i & 0.0911-0.0527 i
\end{array}\right) .
$$

Equally, for an inverted hierarchy we can take a representative point as follows: $\theta_{12}=33.8850^{\circ}, \theta_{23}=42.2823^{\circ}$, $\theta_{13}=8.5649^{\circ}, \quad \delta=244.3791^{\circ}, \rho=48.7884^{\circ}, \sigma=57.5072^{\circ}, \quad m_{1}=0.0644 \mathrm{eV}, m_{2}=0.0650 \mathrm{eV}, \quad m_{3}=0.0421 \mathrm{eV}$, $m_{e}=0.0642 \mathrm{eV}$, and $m_{e e}=0.0623 \mathrm{eV}$ with the corresponding mass matrix (in $\mathrm{eV}$ ):

$$
M_{\nu}=\left(\begin{array}{ccc}
-0.0131+0.0609 i & 0.0099-0.0112 i & 0.0023-0.0022 i \\
0.0099-0.0112 i & 0.0508-0.0098 i & -0.0099+0.0112 i \\
0.0023-0.0022 i & -0.0099+0.0112 i & 0.0507-0.0098 i
\end{array}\right)
$$

We see, from Table II, that $m_{3}$ can reach zero in inverted type, so we expect a possible singular texture existing. Table II also reveals that $J$, at $1-2 \sigma$ levels for both normal and inverted ordering, is negative so the corresponding $\delta$ is in third or fourth quarters. For normal ordering, the ranges for $\rho(\sigma)$ are restricted to be $\left[42^{\circ}, 155^{\circ}\right]\left(\left[17^{\circ}, 102^{\circ}\right]\right)$ at $1 \sigma$ level, whereas they tend to be wider at $3 \sigma$ level covering $\left[6^{\circ}, 175^{\circ}\right]\left(\left[0.01^{\circ}, 180^{\circ}\right]\right)$.

For the plots of Fig. 3 in normal ordering, we find a quasidegenerate mass spectrum where $\left(0.65 \leq m_{23} \leq 0.95\right)$. As to the plots of Fig. 3 in inverted type, we may get an acute hierarchy with $m_{23}$ reaching up to $10^{3}$, so a vanishing $m_{3}$ is possible.

\section{Pattern $\mathrm{C}_{22}$ : Vanishing of $M_{\nu 11}+M_{\nu 33}$}

The relevant expressions for $A_{1}, A_{2}$, and $A_{3}$, as defined in Eq. (12) for this pattern, are

$$
\begin{aligned}
& A_{1}=c_{12}^{2} c_{13}^{2}+\left(c_{12} c_{23} s_{13}-s_{12} s_{23} e^{-i \delta}\right)^{2}, \\
& A_{2}=s_{12}^{2} c_{13}^{2}+\left(s_{12} c_{23} s_{13}+c_{12} s_{23} e^{-i \delta}\right)^{2}, \\
& A_{3}=s_{13}^{2}+c_{23}^{2} c_{13}^{2} .
\end{aligned}
$$

For a representative point with normal ordering, we take $\theta_{12}=33.8006^{\circ}, \theta_{23}=40.7648^{\circ}, \theta_{13}=8.4791^{\circ}, \delta=$ $300.9481^{\circ}, \rho=81.5950^{\circ}, \sigma=69.8454^{\circ}, m_{1}=0.0398 \mathrm{eV}$, $m_{2}=0.0407 \mathrm{eV}, m_{3}=0.0647 \mathrm{eV}, m_{e}=0.0408 \mathrm{eV}$, and $m_{e e}=0.0372 \mathrm{eV}$ with the corresponding mass matrix (in eV):

$$
M_{\nu}=\left(\begin{array}{ccc}
-0.0337+0.0157 i & 0.0064+0.0032 i & 0.0138-0.0059 i \\
0.0064+0.0032 i & 0.0252-0.0235 i & 0.0327+0.0196 i \\
0.0138-0.0059 i & 0.0327+0.0196 i & 0.0337-0.0157 i
\end{array}\right) .
$$

For an inverted hierarchy representative point, we take $\theta_{12}=33.5774^{\circ}, \theta_{23}=42.7607^{\circ}, \theta_{13}=8.7549^{\circ}, \delta=281.0485^{\circ}$, $\rho=99.6048^{\circ}, \sigma=167.4130^{\circ}, m_{1}=0.0749 \mathrm{eV}, m_{2}=0.0754 \mathrm{eV}, m_{3}=0.0571 \mathrm{eV}, m_{e}=0.0747 \mathrm{eV}$, and $m_{e e}=$ $0.0372 \mathrm{eV}$ with the corresponding mass matrix (in $\mathrm{eV}$ ):

$$
M_{\nu}=\left(\begin{array}{ccc}
-0.0263-0.0263 i & 0.0201+0.0479 i & -0.0011-0.0388 i \\
0.0201+0.0479 i & 0.0162+0.0104 i & 0.0336-0.0196 i \\
-0.0011-0.0388 i & 0.0336-0.0196 i & 0.0263+0.0263 i
\end{array}\right) .
$$

We see, from Table II, that $m_{3}$ can reach zero in inverted type, so we expect a possible singular texture existing. Again, from Table II, $J$ at $1-2 \sigma$ levels for normal ordering and $1 \sigma$ for inverted ordering is negative so the corresponding $\delta$ is in third or fourth quarters. For normal ordering, values of $\rho$ are restricted to fall in the range $\left[52^{\circ}, 128^{\circ}\right]$ at the $3 \sigma$ level. 

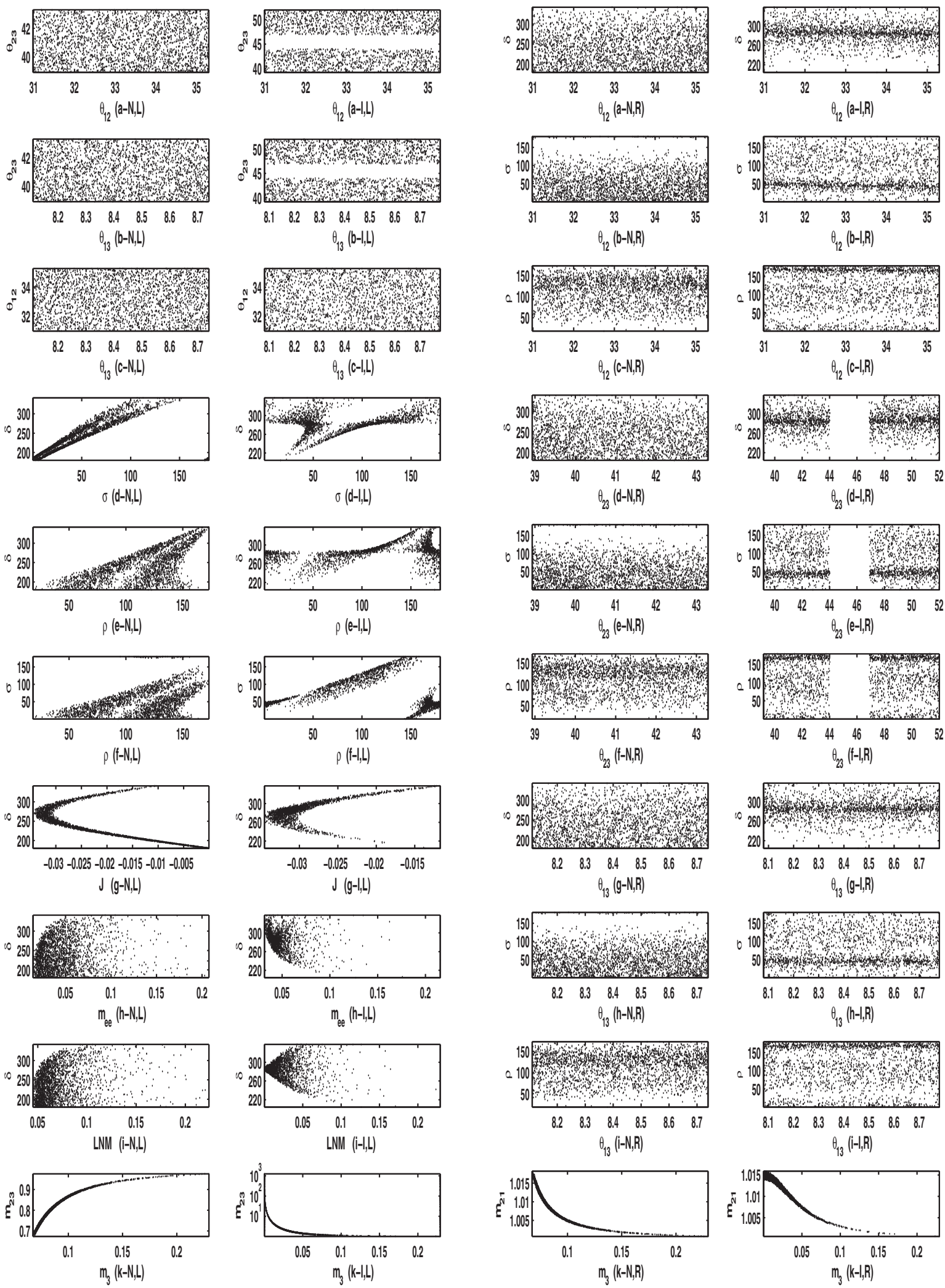

FIG. 3. Pattern $\mathbf{C}_{13} \equiv \mathbf{M}_{\nu 21}+\mathbf{M}_{\nu 23}=0$ for nonsingular mass matrices: The left panel (the left two columns) shows three correlations amidst the mixing angles, three correlations amidst the phase angles, and three correlations of $\delta$ with $J, m_{e e}$, LNM, and finally the correlation $\left(m_{3}, m_{23} \equiv \frac{m_{2}}{m_{3}}\right)$ for normal (N) and inverted (I) hierarchies. The right panel (the right two columns) shows all the nine intercorrelations between phase angles and mixing angles, and the correlation $\left(m_{3}, m_{21} \equiv \frac{m_{2}}{m_{1}}\right)$ for normal (N) and inverted (I) hierarchies. Angles (masses) are evaluated in degrees (eV). 
For the plots, Fig. 4, in normal ordering, we find forbidden bands for $(\sigma, \rho)$ and a moderate mass hierarchy where $\left(0.45 \leq m_{23}<1\right)$. As to the plots of Fig. 4 in inverted type, there are forbidden bands for $(\sigma, \rho)$ and the mass hierarchy can become acute with $m_{23}$ reaching up to $10^{3}$ making a vanishing $m_{3}$ possible.

\section{E. Pattern $\mathrm{C}_{23}$ : Vanishing of $M_{\nu 11}+M_{\nu 23}$}

The relevant expressions for $A_{1}, A_{2}$, and $A_{3}$, as defined in Eq. (12) for this pattern, are

$$
\begin{aligned}
& A_{1}=c_{12}^{2} c_{13}^{2}+\left(c_{12} s_{23} s_{13}+s_{12} c_{23} e^{-i \delta}\right)\left(c_{12} c_{23} s_{13}-s_{12} s_{23} e^{-i \delta}\right), \\
& A_{2}=s_{12}^{2} c_{13}^{2}+\left(s_{12} s_{23} s_{13}-c_{12} c_{23} e^{-i \delta}\right)\left(s_{12} c_{23} s_{13}+c_{12} s_{23} e^{-i \delta}\right), \\
& A_{3}=s_{13}^{2}+s_{23} c_{23} c_{13}^{2} .
\end{aligned}
$$

For a representative point with normal ordering, we take $\theta_{12}=33.4546^{\circ}, \theta_{23}=42.2981^{\circ}, \theta_{13}=8.4653^{\circ}$, $\delta=248.6157^{\circ}, \rho=94.3533^{\circ}, \sigma=68.6630^{\circ}, m_{1}=0.0203 \mathrm{eV}, m_{2}=0.0221 \mathrm{eV}, m_{3}=0.0554 \mathrm{eV}, m_{e}=0.0222 \mathrm{eV}$, and $m_{e e}=0.0175 \mathrm{eV}$ with the corresponding mass matrix (in $\mathrm{eV}$ ):

$$
M_{\nu}=\left(\begin{array}{ccc}
-0.0173+0.0024 i & 0.0012-0.0013 i & 0.0136+0.0007 i \\
0.0012-0.0013 i & 0.0361+0.0029 i & 0.0173-0.0024 i \\
0.0136+0.0007 i & 0.0173-0.0024 i & 0.0369+0.0020 i
\end{array}\right)
$$

For an inverted hierarchy representative point we take $\theta_{12}=33.2679^{\circ}, \theta_{23}=42.8064^{\circ}, \theta_{13}=8.6838^{\circ}, \delta=236.7459^{\circ}$, $\rho=98.4416^{\circ}, \quad \sigma=2.2660^{\circ}, \quad m_{1}=0.0573 \mathrm{eV}, \quad m_{2}=0.0579 \mathrm{eV}, \quad m_{3}=0.0288 \mathrm{eV}, m_{e}=0.0570 \mathrm{eV}$, and $m_{e e}=$ $0.0222 \mathrm{eV}$ with the corresponding mass matrix (in $\mathrm{eV}$ ):

$$
M_{\nu}=\left(\begin{array}{ccc}
-0.0198-0.0100 i & -0.0214+0.0285 i & 0.0299-0.0243 i \\
-0.0214+0.0285 i & 0.0122-0.0172 i & 0.0198+0.0100 i \\
0.0299-0.0243 i & 0.0198+0.0100 i & 0.0042-0.0042 i
\end{array}\right) .
$$

We see, from Table II, that $m_{3}$ cannot reach zero, so we expect no viable corresponding singular pattern. Again, from Table II, $J$ at $1-2 \sigma$ levels for normal ordering and $1 \sigma$ for inverted ordering is negative so the corresponding $\delta$ is in third or fourth quarters. For both normal and inverted ordering, the phase $\rho$ is bound at all $\sigma$ levels to be nearly in the interval $\left(\left[60^{\circ}, 120^{\circ}\right]\right)$.

For the plots, Fig. 5, in both normal and inverted ordering, we get an approximately degenerate spectrum characterized, respectively, by $\left(0.4 \leq m_{23} \leq 0.9\right)$ and $\left(1.2 \leq m_{23} \leq 3\right)$. The plots in Fig. 5 also reveal that the phase $\rho$ is bound to fall approximately in the interval $\left(\left[60^{\circ}, 120^{\circ}\right]\right)$, while there are forbidden bands for the phase $\sigma$ for both types of hierarchies.

\section{F. Pattern $\mathrm{C}_{33}$ : Vanishing of $M_{\nu 11}+M_{\nu 22}$}

The relevant expressions for $A_{1}, A_{2}$, and $A_{3}$, as defined in Eq. (12) for this pattern, are

$$
\begin{aligned}
& A_{1}=c_{12}^{2} c_{13}^{2}+\left(c_{12} s_{23} s_{13}+s_{12} c_{23} e^{-i \delta}\right)^{2}, \\
& A_{2}=s_{12}^{2} c_{13}^{2}+\left(s_{12} s_{23} s_{13}-c_{12} c_{23} e^{-i \delta}\right)^{2}, \\
& A_{3}=s_{13}^{2}+s_{23}^{2} c_{13}^{2} .
\end{aligned}
$$

As for a normal type representative point, we take $\theta_{12}=33.5935^{\circ}, \quad \theta_{23}=40.7528^{\circ}, \quad \theta_{13}=8.7162^{\circ}, \quad \delta=$ $252.1164^{\circ}, \rho=77.1818^{\circ}, \sigma=164.3730^{\circ}, m_{1}=0.0530 \mathrm{eV}$, $m_{2}=0.0537 \mathrm{eV}, m_{3}=0.0736 \mathrm{eV}, m_{e}=0.0537 \mathrm{eV}$, and $m_{e e}=0.0184 \mathrm{eV}$ with the corresponding mass matrix (in $\mathrm{eV}$ ):

$$
M_{\nu}=\left(\begin{array}{ccc}
-0.0170+0.0072 i & 0.0158+0.0354 i & 0.0047-0.0320 i \\
0.0158+0.0354 i & 0.0170-0.0072 i & 0.0456-0.0010 i \\
0.0047-0.0320 i & 0.0456-0.0010 i & 0.0333+0.0073 i
\end{array}\right)
$$

For an inverted type representative point, we can take $\theta_{12}=33.0850^{\circ}, \theta_{23}=42.6054^{\circ}, \theta_{13}=8.7610^{\circ}, \delta=221.0642^{\circ}$, $\rho=123.4419^{\circ}, \sigma=60.2387^{\circ}, m_{1}=0.0540 \mathrm{eV}, m_{2}=0.0547 \mathrm{eV}, m_{3}=0.0231 \mathrm{eV}, m_{e}=0.0537 \mathrm{eV}$, and $m_{e e}=$ $0.0300 \mathrm{eV}$ with the corresponding mass matrix (in $\mathrm{eV}$ ): 

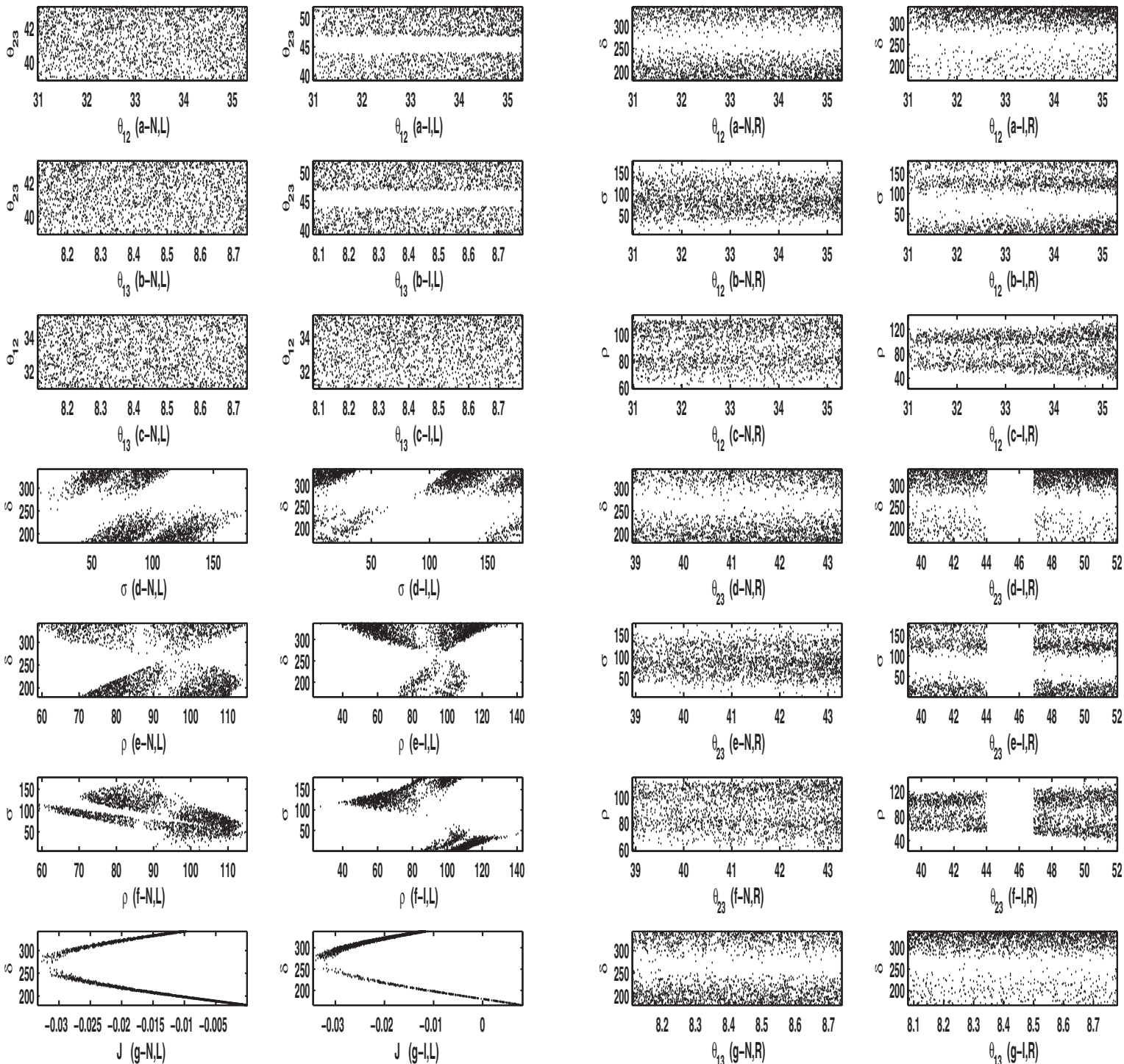

$\theta_{13}(g-N, R)$
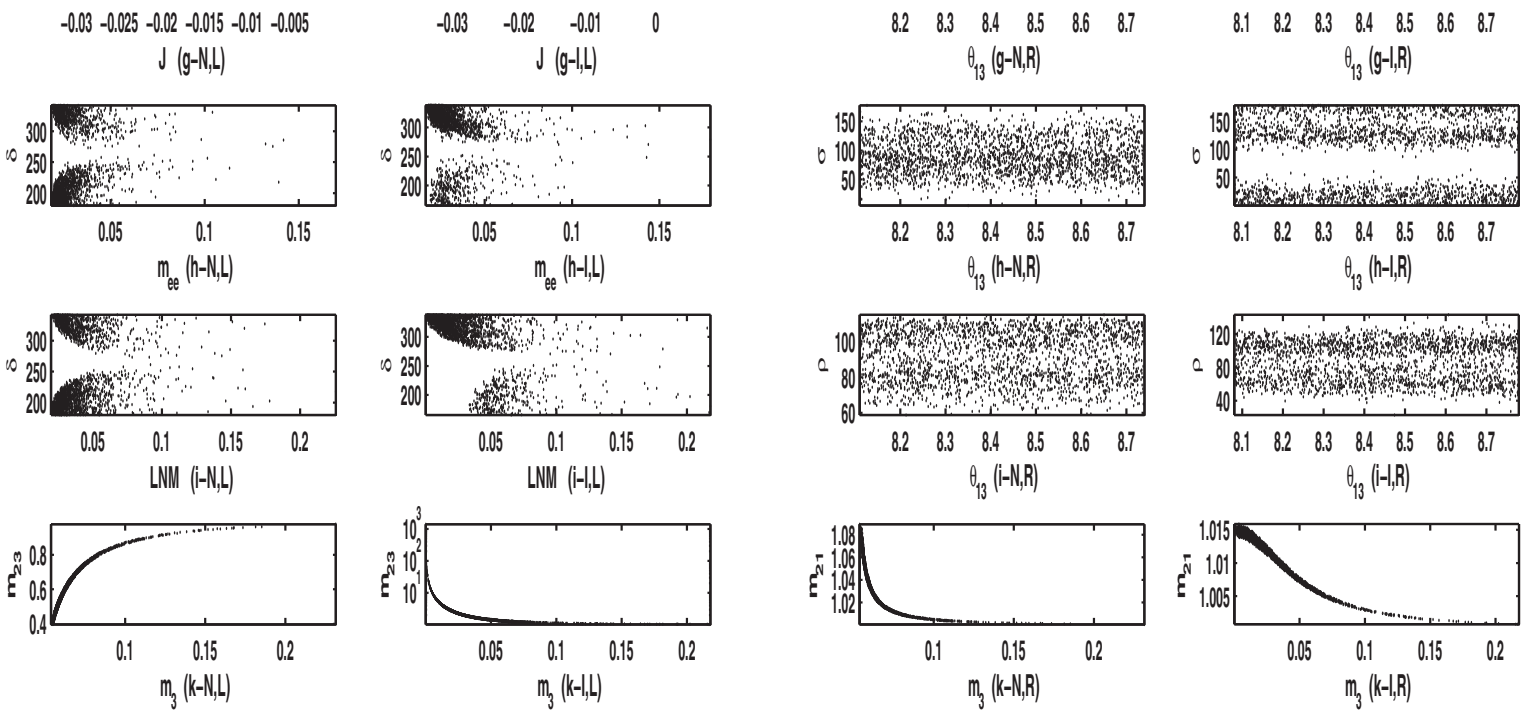

FIG. 4. Pattern $\mathbf{C}_{22} \equiv \mathbf{M}_{\nu 11}+\mathbf{M}_{\nu 33}=0$ for nonsingular mass matrices: The left panel (the left two columns) shows three correlations amidst the mixing angles, three correlations amidst the phase angles, and three correlations of $\delta$ with $J, m_{e e}$, LNM, and finally the correlation $\left(m_{3}, m_{23} \equiv \frac{m_{2}}{m_{3}}\right)$ for normal (N) and inverted (I) hierarchies. The right panel (the right two columns) shows all the nine intercorrelations between phase angles and mixing angles, and the correlation $\left(m_{3}, m_{21} \equiv \frac{m_{2}}{m_{1}}\right)$ for normal (N) and inverted (I) hierarchies. Angles (masses) are evaluated in degrees (eV). 

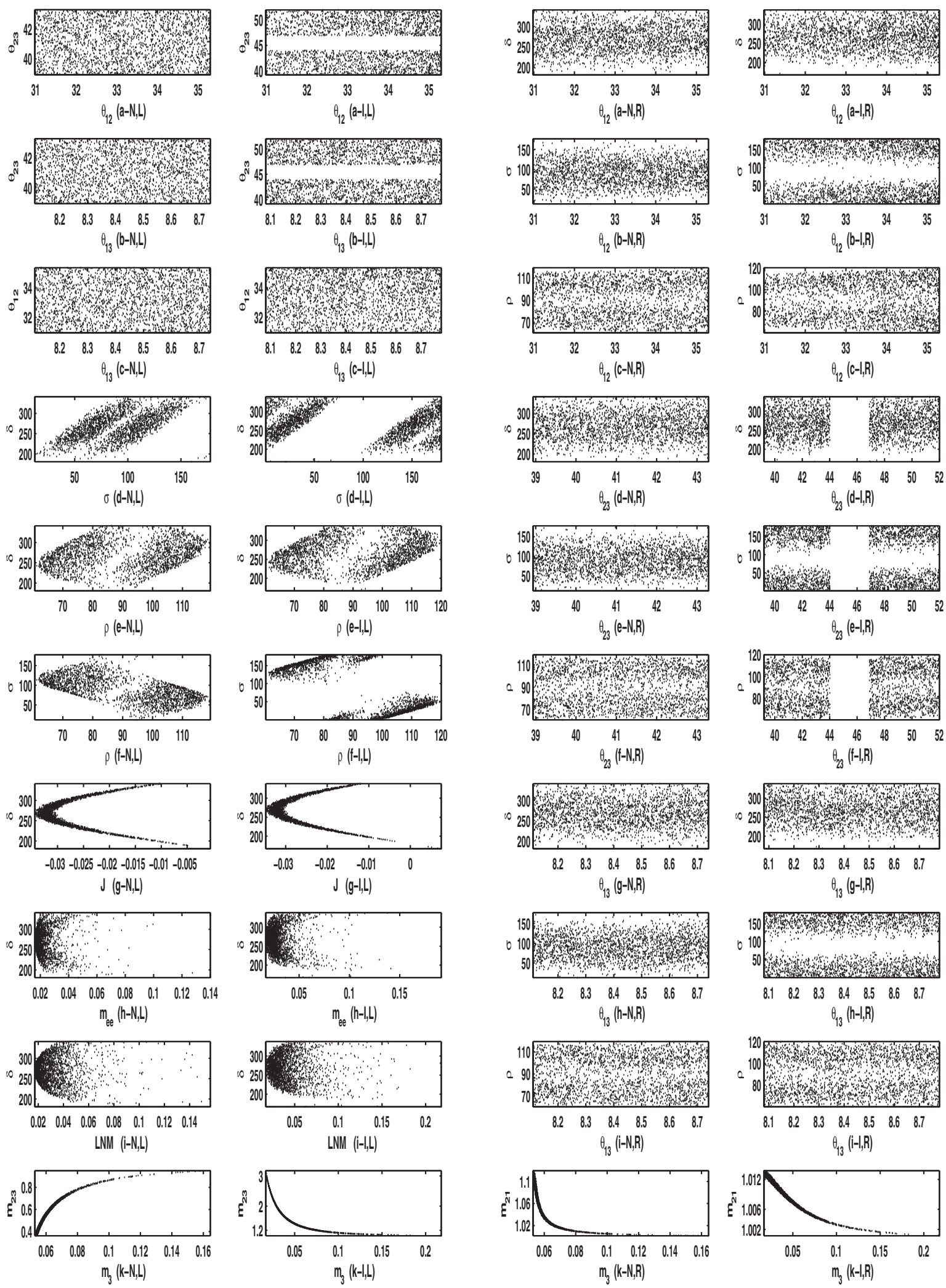

FIG. 5. Pattern $\mathbf{C}_{23} \equiv \mathbf{M}_{\nu 11}+\mathbf{M}_{\nu 23}=0$ for nonsingular mass matrices: The left panel (the left two columns) shows three correlations amidst the mixing angles, three correlations amidst the phase angles, and three correlations of $\delta$ with $J, m_{e e}$, LNM, and finally the correlation $\left(m_{3}, m_{23} \equiv \frac{m_{2}}{m_{3}}\right)$ for normal (N) and inverted (I) hierarchies. The right panel (the right two columns) shows all the nine intercorrelations between phase angles and mixing angles, and the correlation $\left(m_{3}, m_{21} \equiv \frac{m_{2}}{m_{1}}\right)$ for normal (N) and inverted (I) hierarchies. Angles (masses) are evaluated in degrees (eV). 


$$
M_{\nu}=\left(\begin{array}{ccc}
-0.0221-0.0204 i & -0.0148-0.0236 i & 0.0231+0.0260 i \\
-0.0148-0.0236 i & 0.0221+0.0204 i & 0.0041-0.0138 i \\
0.0231+0.0260 i & 0.0041-0.0138 i & 0.0146+0.0072 i
\end{array}\right)
$$

We see, from Table II, that $m_{3}$ can reach zero in inverted type, so we expect a viable corresponding singular pattern. Again, from Table II, $J$ at $1-2 \sigma$ levels for normal ordering and $1 \sigma$ for inverted ordering is negative so the corresponding $\delta$ is in third or fourth quarters. For normal ordering, the values of the phase $\rho$ are restricted to fall in the range $\left[64^{\circ}, 126^{\circ}\right]$ at the $1 \sigma$ level, in $\left[56^{\circ}, 129^{\circ}\right]$ at the $2 \sigma$ level, and in $\left[52^{\circ}, 130^{\circ}\right]$ at the $3 \sigma$ level, but, in contrast, there is almost no restriction for $\sigma$. For inverted ordering, there is a restriction for the phase $\rho$ range: $\left[57^{\circ}, 132^{\circ}\right]$ at $1-\sigma$ level, $\left[15^{\circ}, 170^{\circ}\right]$ at $2-\sigma$ level, and $\left[1.5^{\circ}, 173^{\circ}\right]$ at $3-\sigma$ level. In contrast, there is a forbidden gap for $\sigma$ which is $\left[94^{\circ}, 142^{\circ}\right]$ at $1-\sigma$ level, $\left[86^{\circ}, 106^{\circ}\right]$ at $2-\sigma$ level, and $\left[83^{\circ}, 100^{\circ}\right]$ at $3-\sigma$ level.

For the plots, Fig. 6, in normal ordering, we find narrow forbidden bands for $(\rho)$ and a mild mass hierarchy characterized by $\left(0.35 \leq m_{23} \leq 0.9\right)$. As to the plots, Fig. 6, in inverted type, we also find forbidden bands for both $\rho$ and $\sigma$, but the hierarchy can be severe with $m_{23}$ reaching up to $10^{4}$ indicating the possibility of vanishing $m_{3}$.

\section{PHENOMENOLOGICAL ANALYSIS FOR SINGULAR TEXTURES}

Experimental data allow for one neutrino mass to vanish. Equations (11) are not valid when the neutrino mass matrix is singular, where instead we should use Eqs. (14) and (15) to calculate the mass spectrum given the mixing and phase (Dirac and one Majorana) angles and the solar squared mass splitting. The analytic formulas we get are simpler than when the mass matrix is invertible, but still they are too cumbersome to write them down, even if one restricts to first order in powers of $s_{z}$.

The mass spectrum in the normal ordering is given by

$$
\begin{aligned}
m_{1} & =0, \quad m_{2}=\sqrt{\delta m^{2}}, \quad m_{3}=\sqrt{\Delta m^{2}+\delta m^{2} / 2}, \\
\Delta m^{2} & =\delta m^{2}\left(\left|\frac{A_{2}}{A_{3}}\right|^{2}-\frac{1}{2}\right) .
\end{aligned}
$$

Numerically, no singular texture of normal type could accommodate data.

In the inverted ordering the mass spectrum is given by

$$
\begin{aligned}
& m_{3}=0, \quad m_{1}=\sqrt{\Delta m^{2}-\delta m^{2} / 2}, \\
& m_{2}=\sqrt{\Delta m^{2}+\delta m^{2} / 2}, \quad \Delta m^{2}=\frac{1}{2} \delta m^{2}\left(\frac{\left|\frac{A_{1}}{A_{2}}\right|^{2}+1}{\left|\frac{A_{1}}{A_{2}}\right|^{2}-1}\right) .
\end{aligned}
$$

Four "acceptable" textures $\left(\mathbf{C}_{12}, \mathbf{C}_{13}, \mathbf{C}_{22}, \mathbf{C}_{33}\right)$ are found able to accommodate data.

We follow the same methodology in generating numerical results (random sampling) and the same nomenclature in presenting results as in the case of nonsingular mass matrices. All various predictions concerning the ranges spanned by mixing angles, phase angles, neutrino masses, $m_{e}, m_{e e}$, and $J$ are summarized in Table III. We note that the textures $\mathbf{C}_{22}$ and $\mathbf{C}_{33}$ do not pass the experimental constraints at $1 \sigma$ level. We present for each viable singular texture the neutrino mass matrix obtained at one representative point chosen from the accepted points out of those generated randomly in the corresponding parameter space at the $3-\sigma$ level. The choice of the representative point is made in such a way to be as close as possible to the best fit values for mixing and Dirac phase angles.

Briefly, we see that $J<0$ at all $\sigma$ levels for the texture $\mathbf{C}_{13}$, putting $\delta$ in the third and fourth quarters. The same applies for the texture $\mathbf{C}_{12}$ at $1-2 \sigma$ levels, and for the texture $\mathbf{C}_{22}$ at $2 \sigma$ level, specifying equally the $\delta$ quarters for these acceptable textures. Positive values for $J$ can be achieved at $3 \sigma$ level for the textures $\mathbf{C}_{12}$ and $\mathbf{C}_{22}$ and also at $1-2 \sigma$ levels for the texture $\mathbf{C}_{33}$.

Finally, we plot for each texture the possible correlations at the $2 \sigma$ level showing 18 correlations grouped into two panels. The left panel shows three correlations amidst the mixing angles, three correlations amidst the phase angles, and two correlations of $\delta$ with $\left(J, m_{e e}\right)$, and finally the correlation $\left(m_{12} \equiv m_{1} / m_{2}, m_{2}\right)$. The right panel includes all the nine intercorrelations between phase angles and mixing angles.

In all four acceptable textures, the mass spectrum is almost degenerate $\left(m_{1} \approx m_{2}\right)$, and there is a strong linear correlation between $(\rho, \sigma)$ depicting two linear ribbons of positive slope. Also, there is a linear correlation between $(J, \delta)$ in the four textures, and this is due to the small allowed range for $\delta$ which renders the sine curve $(J \propto \sin \delta)$ looking like a linear one. In this respect, especially clear is the positive (negative) slope in the texture $\mathbf{C}_{22}\left(\mathbf{C}_{33}\right)$.

\section{A. Singular pattern of $\mathrm{C}_{12}$ : Vanishing of $M_{\nu 21}+M_{\nu 33}$ and $m_{3}$}

We see, from Table III, that $J$ is negative at $1-2 \sigma$ levels and the corresponding $\delta$ is in the third quarter.

For a representative point we take with $m_{3}=0$ : $\theta_{12}=33.8683^{\circ}, \quad \theta_{23}=40.9412^{\circ}, \quad \theta_{13}=8.7098^{\circ}, \quad \delta=$ $255.0672^{\circ}, \rho=26.7494^{\circ}, \sigma=174.1569^{\circ}, m_{1}=0.0490 \mathrm{eV}$, $m_{2}=0.0498 \mathrm{eV}, m_{e}=0.0487 \mathrm{eV}$, and $m_{e e}=0.0417 \mathrm{eV}$ with the corresponding mass matrix (in $\mathrm{eV}$ ): 

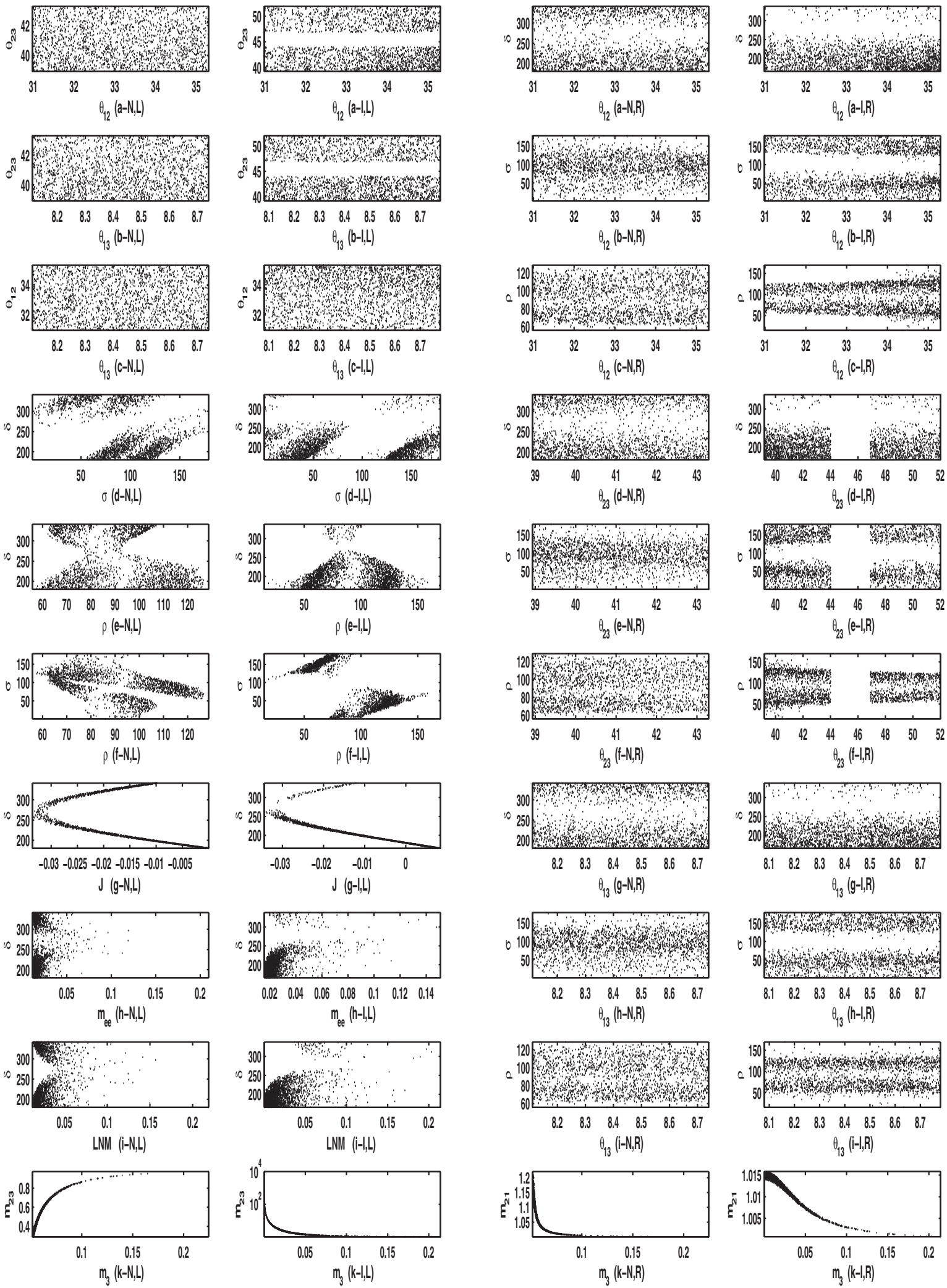

FIG. 6. Pattern $\mathbf{C}_{33} \equiv \mathbf{M}_{\nu 11}+\mathbf{M}_{\nu 22}=0$ for nonsingular mass matrices: The left panel (the left two columns) shows three correlations amidst the mixing angles, three correlations amidst the phase angles, and three correlations of $\delta$ with $J, m_{e e}, \mathrm{LNM}$, and finally the correlation $\left(m_{3}, m_{23} \equiv \frac{m_{2}}{m_{3}}\right)$ for normal (N) and inverted (I) hierarchies. The right panel (the right two columns) shows all the nine intercorrelations phase angles and mixing angles, and the correlation $\left(m_{3}, m_{21} \equiv \frac{m_{2}}{m_{1}}\right)$ for normal (N) and inverted (I) hierarchies. Angles (masses) are evaluated in degrees $(\mathrm{eV})$. 


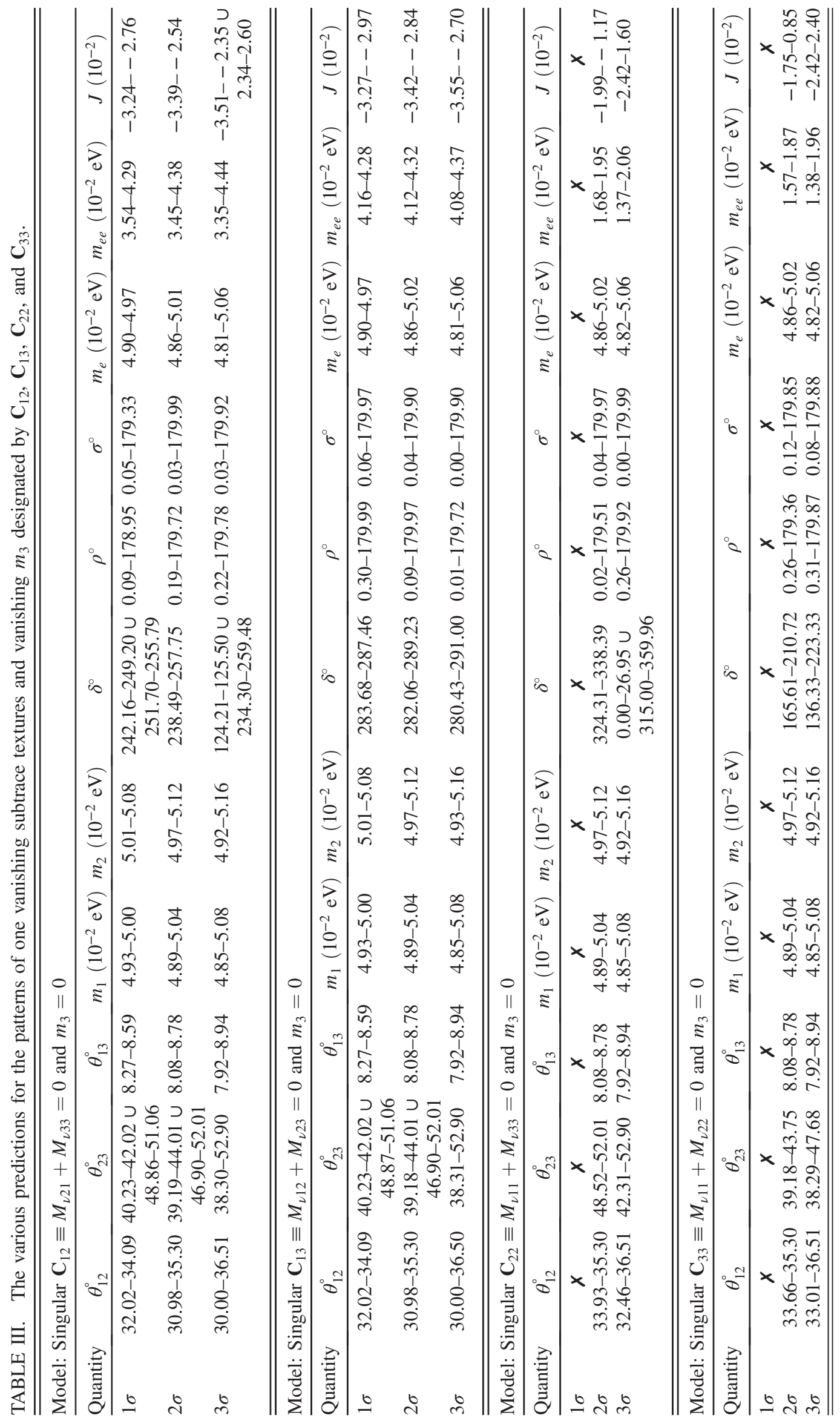




$$
M_{\nu}=\left(\begin{array}{ccc}
0.0344+0.0235 i & 0.0113+0.0086 i & -0.0168-0.0122 i \\
0.0113+0.0086 i & -0.0222-0.0167 i & 0.0170+0.0127 i \\
-0.0168-0.0122 i & 0.0170+0.0127 i & -0.0113-0.0086 i
\end{array}\right)
$$

For the plots, Fig. 7, when $J$ increases $\delta$ tends to decrease in a linear manner. A strong positive linear correlation between $(\rho, \sigma)$ exists with two ribbons. There is a forbidden gap for $m_{e e}:[0.0395,0.0400] \mathrm{eV}$. The mass spectrum is almost degenerate $\left(m_{1} \approx m_{2}\right)$.

\section{B. Singular pattern of $\mathrm{C}_{13}$ : Vanishing of $M_{\nu 12}+M_{\nu 23}$ and $m_{3}$}

We see, from Table III, that $J$ is negative at all levels and the corresponding $\delta$ is in the fourth quarter.

For a representative point we take with $m_{3}=0: \theta_{12}=33.8148^{\circ}, \theta_{23}=40.7781^{\circ}, \theta_{13}=8.4919^{\circ}, \delta=284.0999^{\circ}$, $\rho=53.2226^{\circ}, \sigma=85.4376^{\circ}, m_{1}=0.0496 \mathrm{eV}, m_{2}=0.0504 \mathrm{eV}, m_{e}=0.0493 \mathrm{eV}$, and $m_{e e}=0.0424 \mathrm{eV}$ with the corresponding mass matrix (in $\mathrm{eV}$ ):

$$
M_{\nu}=\left(\begin{array}{ccc}
-0.0246+0.0346 i & 0.0127-0.0187 i & -0.0061+0.0093 i \\
0.0127-0.0187 i & 0.0118-0.0174 i & -0.0127+0.0187 i \\
-0.0061+0.0093 i & -0.0127+0.0187 i & 0.0122-0.0180 i
\end{array}\right)
$$

For the plots in Fig. 8, a linear correlation between $J$ and $\delta$ exists where $J$ tends to increase as $\delta$ increases. The plots in Fig. 8 also reveal a strong linear correlation between $(\rho, \sigma)$ with two narrow ribbons exists. Also, there is a negative-slope linear dependence between $\left(\delta, \theta_{12}\right)$. The neutrino masses are almost degenerate $\left(m_{1} \approx m_{2}\right)$.

\section{Singular pattern of $\mathrm{C}_{22}$ : Vanishing of $M_{\nu 11}+M_{\nu 33}$ and $m_{3}$}

We see, from Table III, that at $1 \sigma$ level, the singular pattern is not viable. We also note that $J$ is negative at $2 \sigma$ level and the corresponding $\delta$ is in the fourth quarter.

For a representative point we take with $m_{3}=0: \theta_{12}=34.5161^{\circ}, \theta_{23}=50.8655^{\circ}, \theta_{13}=8.5346^{\circ}, \delta=339.6445^{\circ}$, $\rho=124.3227^{\circ}, \sigma=26.7978^{\circ}, m_{1}=0.0492 \mathrm{eV}, m_{2}=0.0499 \mathrm{eV}, m_{e}=0.0489 \mathrm{eV}$, and $m_{e e}=0.0180 \mathrm{eV}$ with the corresponding mass matrix (in $\mathrm{eV}$ ):

$$
M_{\nu}=\left(\begin{array}{ccc}
-0.0026-0.0178 i & 0.0046+0.0304 i & -0.0050-0.0331 i \\
0.0046+0.0304 i & 0.0000+0.0007 i & -0.0011-0.0081 i \\
-0.0050-0.0331 i & -0.0011-0.0081 i & 0.0026+0.0178 i
\end{array}\right) .
$$

For the plots in Fig. 9, $J$ and $\delta$ are correlated quasilinearly and positively. A strong linear correlation with two ribbons between $(\rho, \sigma)$ exists. The mass spectrum is almost degenerate $\left(m_{1} \approx m_{2}\right)$.

\section{Singular pattern of $\mathrm{C}_{33}$ : Vanishing of $M_{\nu 11}+M_{\nu 22}$ and $m_{3}$}

As in the previous case $\mathbf{C}_{22}$, the singular pattern $\mathbf{C}_{33}$ is not viable at $1 \sigma$ level as evident from Table III.
In contrast to the previous case $\mathbf{C}_{22}, J$ can assume positive as well as negative values at $2 \sigma$ level and the corresponding $\delta$ lies in the second and third quarters.

For a representative point we take with $m_{3}=0$ : $\theta_{12}=35.9702^{\circ}, \quad \theta_{23}=42.1759^{\circ}, \quad \theta_{13}=8.4675^{\circ}, \quad \delta=$ $204.6858^{\circ}, \rho=127.4906^{\circ}, \sigma=45.5467^{\circ}, m_{1}=0.0487 \mathrm{eV}$, $m_{2}=0.0495 \mathrm{eV}, m_{e}=0.0485 \mathrm{eV}$, and $m_{e e}=0.0159 \mathrm{eV}$ with the corresponding mass matrix (in $\mathrm{eV}$ ):

$$
M_{\nu}=\left(\begin{array}{ccc}
-0.0084-0.0135 i & -0.0169-0.0275 i & 0.0170+0.0276 i \\
-0.0169-0.0275 i & 0.0084+0.0135 i & -0.0042-0.0067 i \\
0.0170+0.0276 i & -0.0042-0.0067 i & 0.0004+0.0005 i
\end{array}\right) .
$$

For the plots in Fig. 10, we see that $(J, \delta)$ are strongly correlated linearly and negatively. A strong linear correlation between $(\rho, \sigma)$ exists with two ribbons. The masses $\left(m_{1}, m_{2}\right)$ are almost degenerate. 

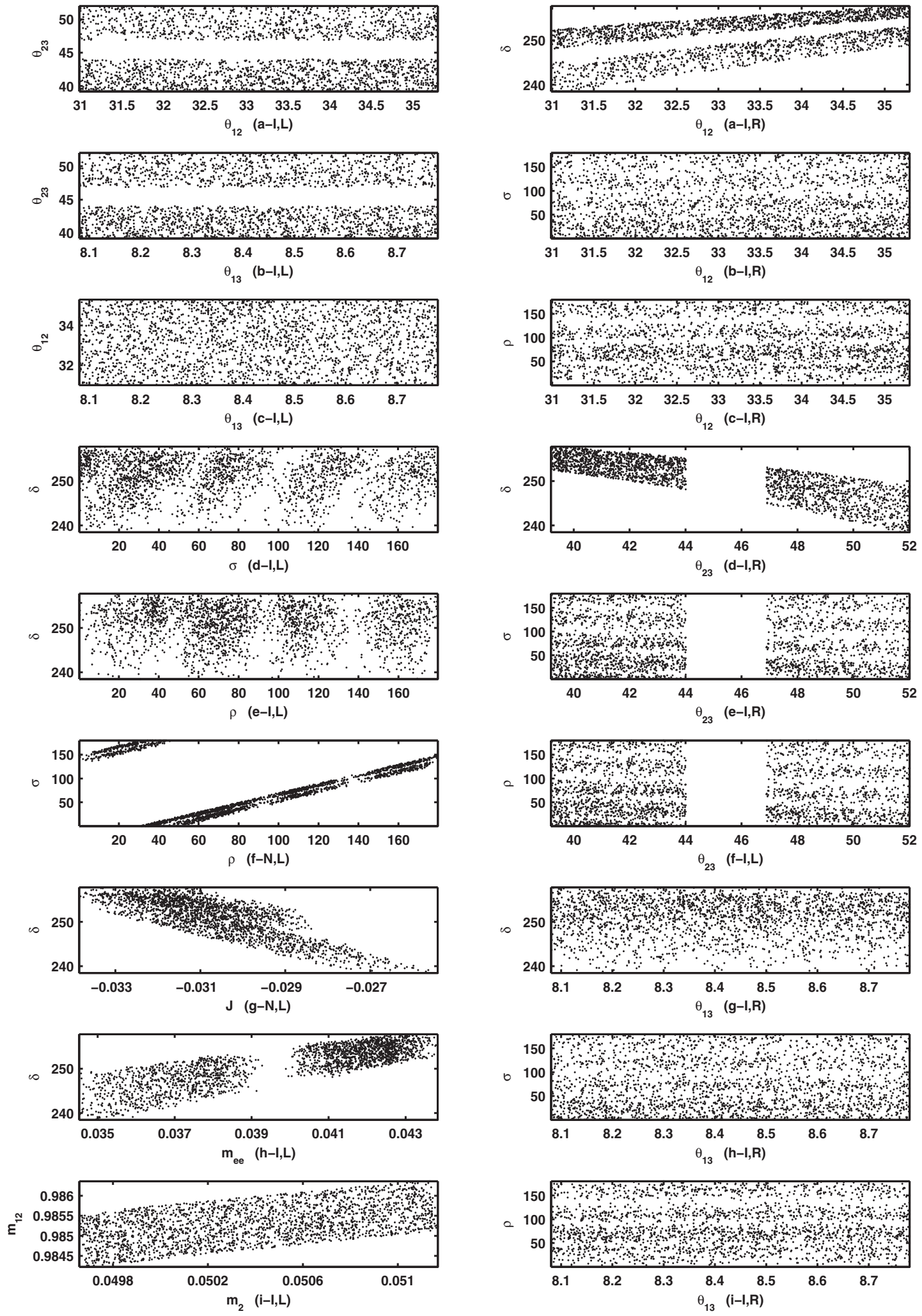

FIG. 7. Pattern $\mathbf{C}_{12}$ for singular mass matrices with inverted ordering: The left panel shows three correlations amidst the mixing angles, three correlations amidst the phase angles, and two correlations of $\delta$ with $J, m_{e e}$, and finally the correlation $\left(m_{12} \equiv \frac{m_{1}}{m_{2}}, m_{2}\right)$. The right panel shows all the nine correlations interphase angles and mixing angles. Angles (masses) are evaluated in degrees $(\mathrm{eV})$. 

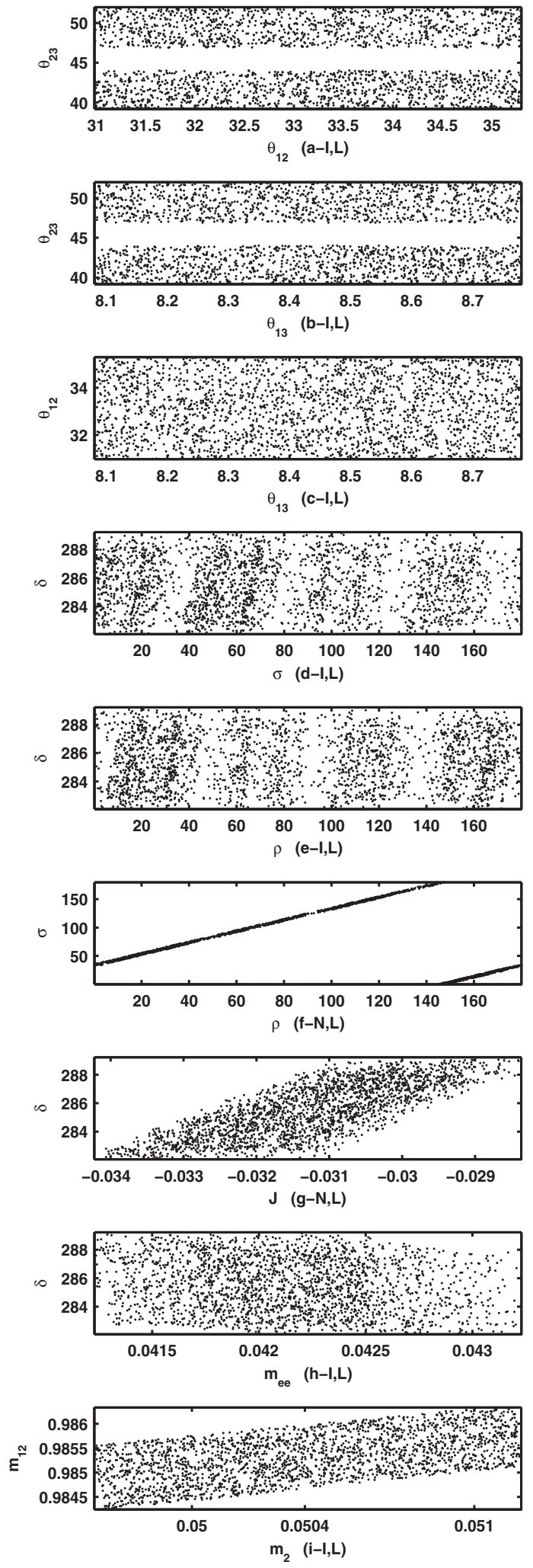
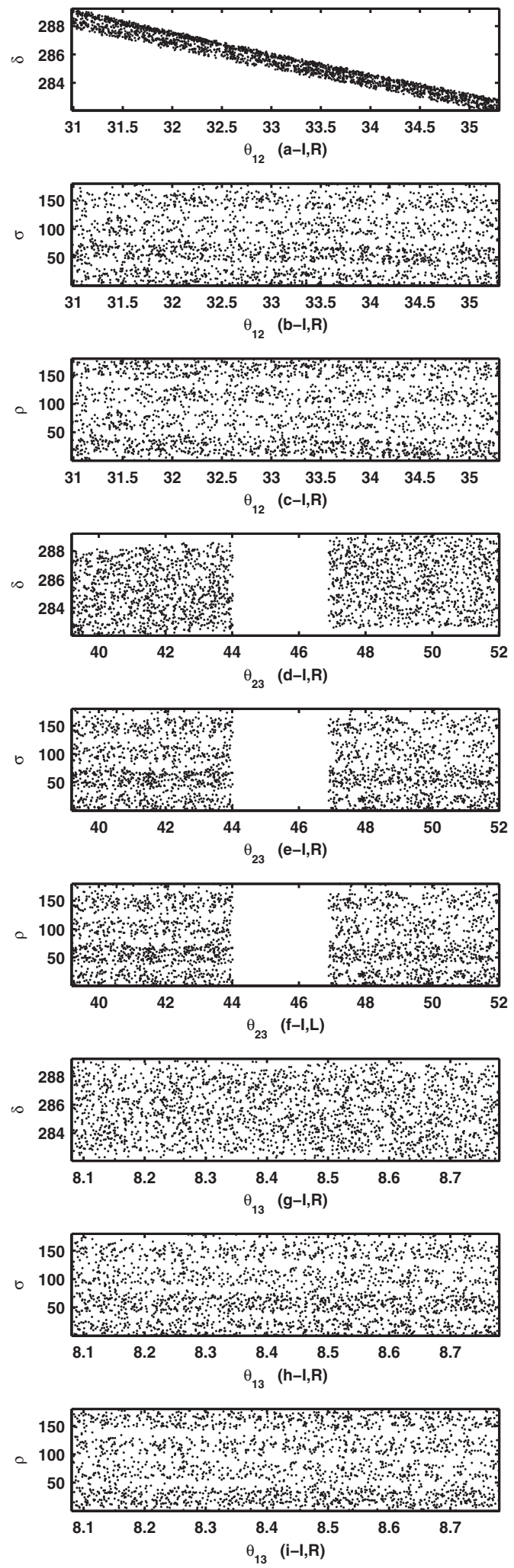

FIG. 8. Pattern $\mathbf{C}_{13}$ for singular mass matrices with inverted ordering: The left panel shows three correlations amidst the mixing angles, three correlations amidst the phase angles, and two correlations of $\delta$ with $J, m_{e e}$, and finally the correlation $\left(m_{12} \equiv \frac{m_{1}}{m_{2}}, m_{2}\right)$. The right panel shows all the nine correlations between phase angles and mixing angles. Angles (masses) are evaluated in degrees $(\mathrm{eV})$. 

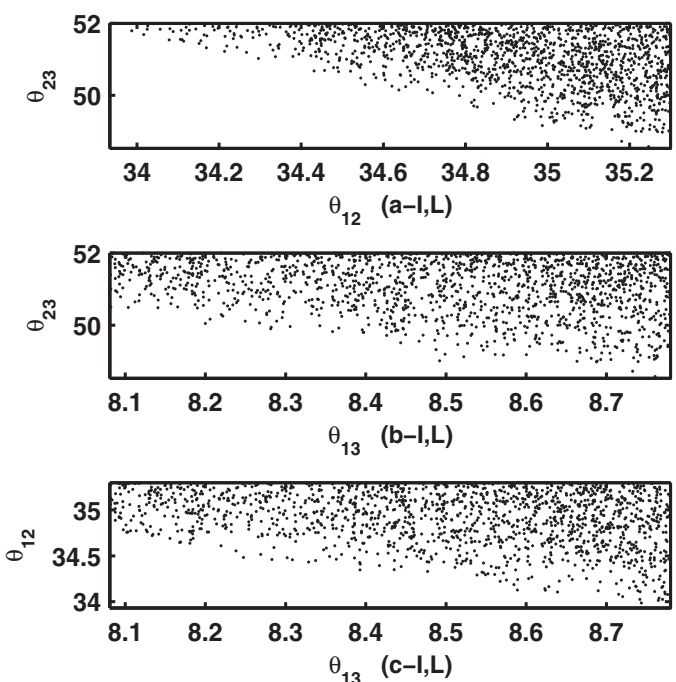

$\infty$

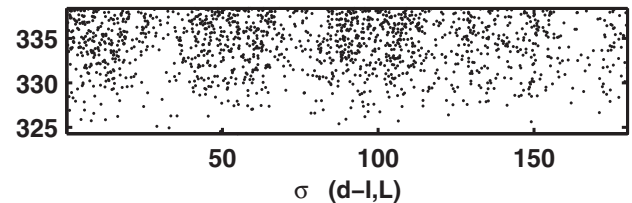

$\infty$

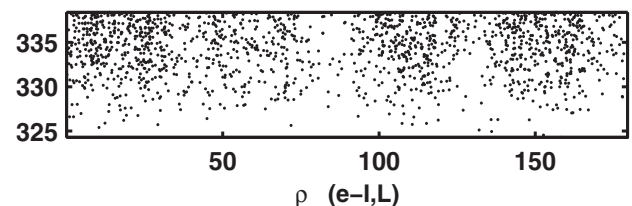

0
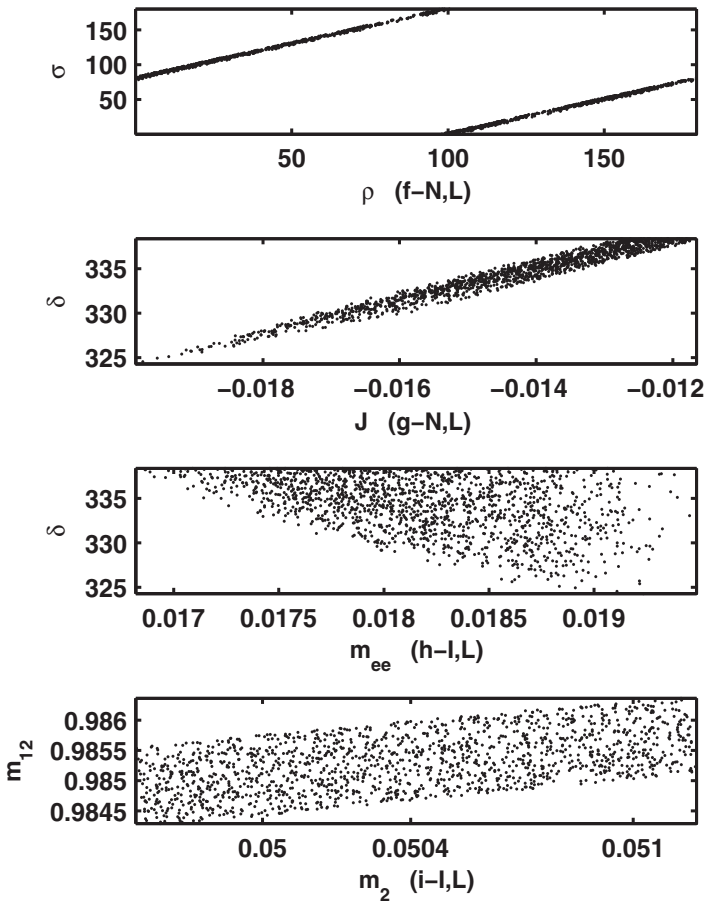
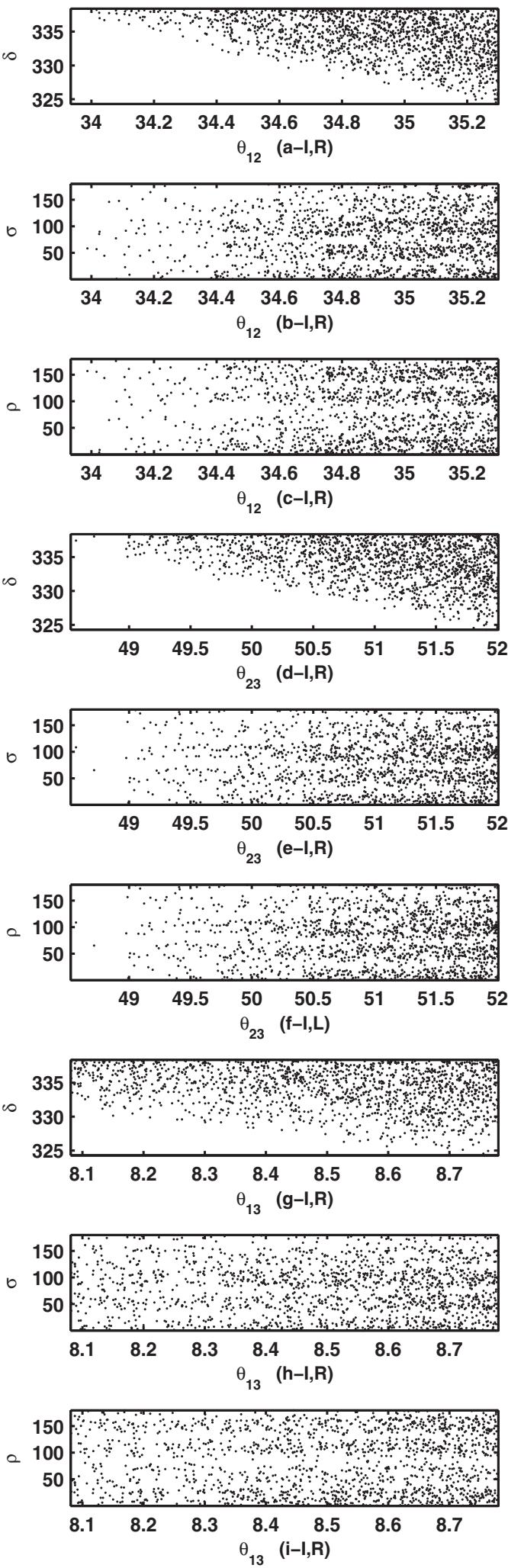

FIG. 9. Pattern $\mathbf{C}_{22}$ for singular mass matrices with inverted ordering: The left panel shows three correlations amidst the mixing angles, three correlations amidst the phase angles, and two correlations of $\delta$ with $J, m_{e e}$, and finally the correlation $\left(m_{12} \equiv \frac{m_{1}}{m_{2}}, m_{2}\right)$. The right panel shows all the nine correlations between phase angles and mixing angles. Angles (masses) are evaluated in degrees $(\mathrm{eV})$. 

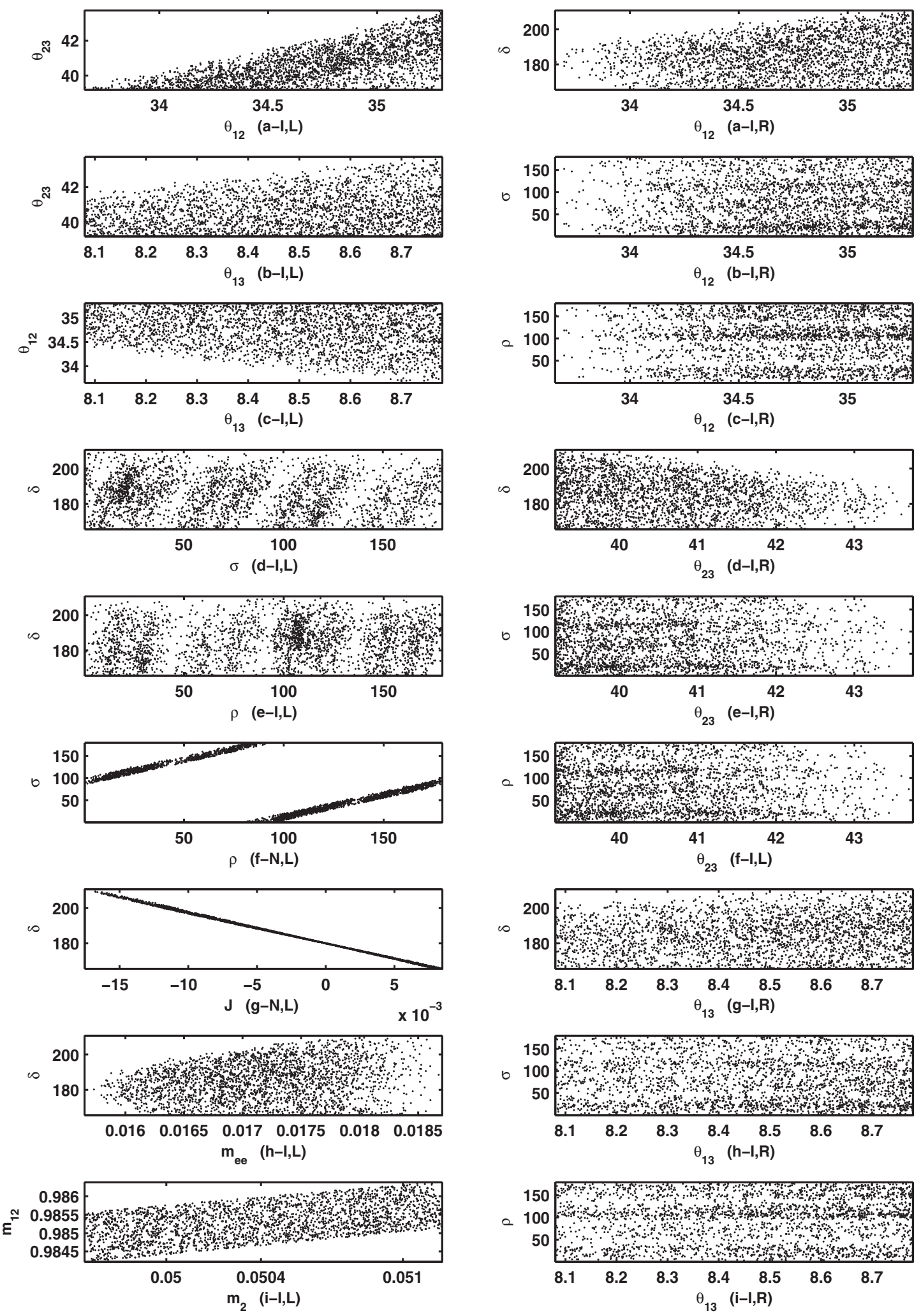

FIG. 10. Pattern $\mathbf{C}_{33}$ for singular mass matrices with inverted ordering: The left panel shows three correlations amidst the mixing angles, three correlations amidst the phase angles, and two correlations of $\delta$ with $J, m_{e e}$, and finally the correlation $\left(m_{12} \equiv \frac{m_{1}}{m_{2}}, m_{2}\right)$. The right panel shows all the nine correlations interphase angles and mixing angles. Angles (masses) are evaluated in degrees (eV). 


\section{THEORETICAL REALIZATION OF THE TEXTURES}

We present in this section theoretical realizations of some of the one vanishing subtrace textures, where symmetry assignments at high scale impose this texture in the "gauge" basis. However, one way to find these assignments is to start from another symmetry imposing zero texture and relate these two symmetries by a rotation. As to the symmetry responsible for imposing the zero elements at high scale, we can just follow the analysis of [5]. We shall find that four vanishing subtrace textures, out of six, are able to be amended by "rotating" zero textures. In Sec. VII, we explain the general strategy of relating the two symmetries, which would be of great help in this method of indirect realization. In Sec. VIII, we discuss the notion of flavor basis due to its paramount relevance into our study. In Sec. IX, making use of rotating zero textures, we adopt a type I seesaw scenario with discrete symmetry $\left(Z_{8} \times Z_{2}\right)$ in order to generate nonsingular vanishing subtrace textures. We repeat the work for singular vanishing subtrace textures in Sec. $X$, but with discrete symmetry $\left(Z_{12} \times Z_{2}\right)$. In Sec. XI, we present an implementation of one vanishing subtrace texture using the type II seesaw scenario supplemented with $\left(Z_{5}\right)$ discrete symmetry, and following the same strategy of "rotation" from zero textures to vanishing subtrace. In Sec. XII, we present a direct way of realization for type I seesaw scenario implementation with $\left(Z_{6} \times Z_{2}\right)$ discrete symmetry not related to zero textures. In Sec. XIII, we pursue the direct method of realization but now for type II seesaw scenario implementation with $\left(Z_{2}^{\prime} \times Z_{2}\right)$ discrete symmetry. One can consider these outlined sections as an exercise in model building aiming to show that the studied texture of vanishing subtrace can be generated at the Lagrangian level by symmetry considerations in which the symmetry is exact but broken spontaneously. The two "indirect" and "direct" methods are on equal footing, and one should not discriminate one against the other. It is just that the fields assignments in the indirect method turn out to be more complex, so we sought a "mathematical" method in order to find them. As a final remark, the presented method of rotation is applicable to any specific pattern that can be generated from the zero-texture pattern via a unitary transformation.

\section{ROTATING STRATEGY: FROM ZERO-TEXTURE TO VANISHING SUBTRACE TEXTURE}

We need to find a unitary matrix $S$ which when acted on the symmetric neutrino matrix,

$$
M_{\nu}=\left(\begin{array}{lll}
A & B & C \\
B & D & E \\
C & E & F
\end{array}\right),
$$

gives the combination that defines the subtrace patterns $\left[\left(\mathbf{C}_{11}\right): D+F\right],\left[\left(\mathbf{C}_{12}\right): B+F\right],\left[\left(\mathbf{C}_{13}\right): B+E\right]$, $\left[\left(\mathbf{C}_{22}\right): A+F\right], \quad\left[\left(\mathbf{C}_{23}\right): A+E\right]$, and $\left[\left(\mathbf{C}_{33}\right): A+D\right]$ in one of the elements of the transformed matrix $\left(\tilde{M}=S^{T} M_{\nu} S\right)$, where $M_{\nu}$ is the effective Majorana neutrino mass matrix. More specifically, for the texture $\mathbf{C}_{33}$, if we take the unitary matrix

$$
S_{33}=\frac{1}{\sqrt{2}}\left(\begin{array}{ccc}
i & -1 & 0 \\
i & 1 & 0 \\
0 & 0 & \sqrt{2}
\end{array}\right),
$$

then we find that

$$
\begin{aligned}
& S_{33} M_{\nu} S_{33}^{T} \\
& \quad=-\frac{1}{2}\left(\begin{array}{ccc}
A+2 i B-D & A+D & -\sqrt{2}(i C-E) \\
A+D & A-2 i B-D & -\sqrt{2}(i C+E) \\
-\sqrt{2}(i C-E) & -\sqrt{2}(i C+E) & -2 F
\end{array}\right),
\end{aligned}
$$

and so the combination $(A+D)$ appears in the element (12) of the transformed matrix

$$
M_{\nu 0}=S_{33} M_{\nu} S_{33}^{T} .
$$

Thus, if by some symmetry $S_{Y 0}$ applied on the transformed matrix $M_{\nu 0}$ one can impose a zero element,

$$
S_{Y 0}^{T} M_{\nu 0} S_{Y 0}=M_{\nu 0} \Rightarrow M_{\nu 0,12}=0
$$

then we see that we have

$$
S_{Y}^{T} M_{\nu} S_{Y}=M_{\nu} \Rightarrow M_{\nu 11}+M_{\nu 22}=0,
$$

where the new symmetry implementing the vanishing subtrace of the texture $C_{33}$ is

$$
S_{Y}=S_{33}^{T} S_{Y 0} S_{33}^{T \dagger} .
$$

Let us define $u^{i j}$ as the matrix resulting by swapping the $i$ th and the $j$ th columns of the identity matrix $(I)$. Then we have the properties

$$
u^{i j}=u^{i j T}=u^{i j \dagger}, \quad u^{i j} u^{i j \dagger}=I .
$$

Then, for any matrix $M$, we see that $\left(M u^{i j}\right)$ swaps the $i$ th and the $j$ th columns of $(M)$, whereas $\left(u^{i j} M\right)$ swaps the $i$ th and the $j$ th rows of $(M)$. Note that $u^{i j} M u^{i j}$ has the effect of swapping first the ( $i$ th and the $j$ th) columns, followed by the ( $i$ th and the $j$ th) rows, or the other way round. We note now that the six vanishing one subtrace textures can be divided into three classes: 
(i) Class of textures $\left\{\mathbf{C}_{11}, \mathbf{C}_{22}, \mathbf{C}_{33}\right\}$ : In the sense that if I find a unitary transformation $\tilde{S}$ giving me one of them, then I directly get the unitary transformation giving me the other two textures. This comes because

$$
\left.\begin{array}{l}
\left(u^{13} M u^{13}\right)_{22}=M_{22},\left(u^{13} M u^{13}\right)_{11}=M_{33} \quad \Rightarrow \quad u^{13} \tilde{S}_{33} u^{13}=\tilde{S}_{11}, \\
\left(u^{12} M u^{12}\right)_{33}=M_{33},\left(u^{12} M u^{12}\right)_{11}=M_{22} \quad \Rightarrow \quad u^{12} \tilde{S}_{22} u^{12}=\tilde{S}_{11},
\end{array}\right\}
$$

where $\tilde{S}_{i j}$ is a unitary matrix which, provided its action on $M_{\nu}$ keeps the latter invariant, imposes the texture defined by the subtrace $\left(C_{i, j}\right)$ [see Eq. (45) where $S_{Y}$ plays the role of $\tilde{S}_{33}$ ].

(ii) Class of textures $\left\{\mathbf{C}_{13}\right\}$ : Actually, we can take

$$
S_{13}=\frac{1}{\sqrt{2}}\left(\begin{array}{ccc}
1 & 0 & 1 \\
0 & \sqrt{2} & 0 \\
-1 & 0 & 1
\end{array}\right),
$$

because the $(B+E)$ combination appears in the $(1,2)$ element of

$$
S_{13} M_{\nu} S_{13}^{T}=\frac{1}{2}\left(\begin{array}{ccc}
A+2 C+F & \sqrt{2}(B+E) & F-A \\
\sqrt{2}(B+E) & 2 D & \sqrt{2}(E-B) \\
F-A & \sqrt{2}(E-B) & A-2 C+F
\end{array}\right) .
$$

(iii) Class of textures $\left\{\mathbf{C}_{12}, \mathbf{C}_{32}\right\}$ :

$$
\left(u^{13} M u^{13}\right)_{33}=M_{11}, \quad\left(u^{13} M u^{13}\right)_{21}=M_{23} \Rightarrow u^{13} \tilde{S}_{12} u^{13}=\tilde{S}_{32} .
$$

However, one can algebraically show that the transformation $S M_{\nu} S^{T}$ cannot bring in the sole combination $A+E$, corresponding to the texture $\mathbf{C}_{32}$, at any entry.

Our strategy for the realization of the vanishing subtrace texture is that one imposes a starting symmetry, with corresponding transformations on the Higgs and the lepton fields at the Lagrangian level ("gauge" basis), known to impose some zero elements for the neutrino mass matrix $M_{\nu 0}$. We then transform this symmetry by applying some rotation so that to get a new second symmetry, with new transformations on the fields (also at the gauge basis), which would imply the vanishing subtrace texture for $M_{\nu}$. We stress here that $M_{\nu}$ and $M_{\nu 0}$ are not mass matrices for the same system at different bases related by rotation. Rather, they are mass matrices of two systems, satisfying two different symmetries, where the matrices are defined in the same Lagrangian gauge - or "symmetry"-bases. The two symmetries are related by rotation. By following the previous discussion, we may find the rotation which, when applied on the neutrino mass matrix, allows going from zero texture to vanishing subtrace, so now this rotation would help to define, by Eq. (46), how to move from the first symmetry field transformations to the second symmetry ones by the following "adjoint action" rule:

$$
T_{f}=S^{T} T_{f}^{0} S^{T^{\dagger}},
$$

where $T_{f}\left(T_{f}^{0}\right)$ defines the transformation on the field $f$ satisfying the new (old) symmetry and $S$ is the unitary transformation relating the two symmetries. We remind the reader here that this rotation method is just to find some "complex" field assignments by relating them to other more "trivial" ones, and had we been able to "guess" the complicated assignments, then we would have dispensed with the whole idea of rotation.

However, one should be sure that the new symmetry transformations assure that we are at the flavor basis, or approximately so. The point is that we should get a generic charged lepton mass matrix by the first symmetry, so that we can also get a generic one by the second symmetry. Then, by adopting some natural assumptions on the fields' vacuum expectation values (VEVs), without the need of unnatural constraints on the Yukawa couplings, one can diagonalize the "generic" charged lepton mass matrix by an infinitesimal rotation, and so one can, with a good approximation, assume that the new symmetry puts us in the flavor basis. We shall give some examples for this strategy within both type I and II seesaw scenarios, where the symbol 0 will be consigned for quantities corresponding to the first "unrotated" 
symmetry. ${ }^{1}$ The method based on rotated symmetry can be considered as an indirect method for realizing the texture of the vanishing subtrace.

\section{FLAVOR BASIS}

The notion of basis is intricate and needs to be clarified since a variety of bases could arise in our discussion such as gauge, flavor, and mass basis. In order to delve into the notion of different types of basis, we take for simplicity the following Lagrangian piece responsible for the mass terms in the leptonic sector expressed in the gauge basis as

$$
\begin{aligned}
\mathcal{L}_{M} \supset & Y_{1 i j}^{g} \bar{D}_{L i} H e_{R j}+Y_{2 i j}^{g} \bar{D}_{L i} \tilde{H} \nu_{R j} \\
& +Y_{3 i j}^{g} \nu_{R i}^{T} \mathcal{C}^{-1} \chi \nu_{R j},
\end{aligned}
$$

where $D_{L i}$ is the left-handed lepton doublet $\left(\nu_{L i}, e_{L i}\right)^{T}, e_{R i}$ is the right-handed charged lepton, $\nu_{R i}$ is the right-handed neutrino, $\chi$ is a scalar singlet, $H$ is the Higgs doublet, and $\tilde{H} \equiv i \sigma_{2} H^{*}$. The relevant Yukawa coupling matrices are denoted by $\left(Y_{1}^{g}, Y_{2}^{g}, Y_{3}^{g}\right)$ which are defined in this "gauge" basis, whence the superscript $(g)$. The indices $i, j$ are the family ones while $\mathcal{C}$ is the charge conjugation.

When the Higgs doublet $H$ and the singlet $\chi$ take a VEV, then we get the mass term which can be cast into the form

$$
\begin{aligned}
& \bar{e}_{L i} M_{\ell i j} e_{R j}+\bar{e}_{R i} M_{\ell i j} e_{L j} \\
& \quad+\left(\begin{array}{ll}
\nu_{L i}^{T} & \nu_{R j}^{T}
\end{array}\right) \mathcal{C}^{-1}\left(\begin{array}{cc}
0 & M_{D i j} \\
M_{D j i} & M_{R i j}
\end{array}\right)\left(\begin{array}{l}
\nu_{L i} \\
\nu_{R j}
\end{array}\right),
\end{aligned}
$$

which, via the seesaw mechanism, gives approximately, after decoupling the right-handed neutrinos,

$$
\mathcal{L}_{M} \supset \bar{e}_{L_{i}} M_{\ell i j} e_{R j}+\nu_{l i}^{T} \mathcal{C}^{-1} M_{\nu i j} \nu_{l j}
$$

with $M_{\nu}=M_{D} M_{R}^{-1} M_{D}^{T}$, and $\nu_{l i}$ are approximately lefthanded $\left(\approx \nu_{L i}\right)$.

By diagonalizing, we get the "mass" basis which is denoted by the superscript $m$ :

$$
\left.\begin{array}{rl}
\mathcal{L}_{M} & \supset \bar{e}_{L}^{m} U_{L}^{\dagger} M_{\ell} U_{R} e_{R}^{m}+\nu_{l}^{m} V^{T} M_{\nu} V \nu_{l}^{m}, \\
& \supset \bar{e}_{L}^{m} M_{\ell}^{\mathrm{diag}} e_{R}^{m}+\nu_{l}^{m} M_{\nu}^{\mathrm{diag}} \nu_{l}^{m}, \\
e_{L}^{m} & =U_{L}^{\dagger} e_{L}, e_{R}^{m}=U_{R}^{\dagger} e_{R}, \nu_{l}^{m}=V^{\dagger} \nu_{l},
\end{array}\right\}
$$

where $U_{L}^{\dagger} M_{\ell} U_{R}$ and $V^{T} M_{\nu} V$ are diagonal.

\footnotetext{
${ }^{1}$ Concretely, a discrete symmetry of the form $Z_{n}^{0} \times Z_{m}^{0}$ is imposed on one system leading to zero textures in the neutrino mass matrix $M_{\nu 0}$ in the "symmetry-gauge" basis, whereas another system has a symmetry $Z_{n} \times Z_{m}$ leading to a vanishing subtrace in the corresponding $M_{\nu}$ defined again in the symmetry-gauge basis. The unitary transformation $S$ helps to relate the field assignments of both systems under the two symmetries.
}

In the gauge basis, the interaction (say, $\bar{e}_{L} W^{-} \nu_{l}$ ) between the charged lepton sector and the neutrino sector, when expressed in terms of the mass bases $\left(\bar{e}_{L}^{m} U_{L}^{\dagger} V W^{-} \nu_{l}^{m}\right)$ would involve the experimentally measurable $V_{\mathrm{PMNS}}=U_{L}^{\dagger} V$ expressing the mismatch between the rotations of the left-handed charged leptons and of the left-handed neutrinos. The "flavor basis," by definition, occurs when by convention we assume, without loss of generality, the lefthanded charged leptons to be pure states, i.e., $U_{L}=1$ and $e_{L}^{m}=e_{L}$. This can always be taken, since one can use the freedom in defining the fields in a way to attribute the whole rotation, appearing when expressing the interaction term in terms of mass states, entirely to the left-handed neutrinos. The situation is exactly the same for the quark sectors when one can take by convention the up sector as pure states and the flavor mixing is described in terms of the rotation Cabibbo-Kobayashi-Maskawa matrix operating on the down sector only [17].

In the realization models we shall construct in the next sections, the field assignments are given in the symmetrygauge basis at the level of the Lagrangian, and thus we get a charged lepton field mass matrix which is not necessarily diagonal. We shall examine at which conditions one can have diagonal, or almost diagonal to a very good approximation, mass matrix for the charged leptons, in a way to say that the symmetry leading to the sought for texture in the neutrino sector puts us also, nearly, in the flavor basis for the charged leptons.

The question arises as to whether one should update the phenomenological analysis upon carrying out the infinitesimally small, under these conditions, rotation $R_{\epsilon}$ of the charged leptons from the symmetry-gauge into the "flavor (mass)" basis. Actually, the phenomenology study was carried out in the flavor basis, which means it is valid up to small corrections of the order of the small rotation $R_{\epsilon}{ }^{2}$ With this in mind, this small correction should be added to the already anticipated one stemming from the renormalization group loop effects upon running from the high scale, when the symmetry was imposed, to the low scale of the experimental data.

\section{INDIRECT REALIZATION OF TYPE I SEESAW WITH $Z_{8} \times Z_{2}$ SYMMETRY FOR NONSINGULAR TEXTURES}

We implement here a discrete symmetry within a type I seesaw scenario in order to generate one vanishing subtrace texture following the rotating strategy.

\footnotetext{
${ }^{2}$ One should be aware not to mix the two rotations. The "mathematical rotation" $S$ relating two systems with two different discrete symmetries, which is "large (finite)" normally, and the "physical rotation" $R_{\epsilon}$, which is "small (infinitesimal)," and is applied on the charged leptons to go from the symmetry-gauge basis into the flavor-mass basis.
} 


\section{A. Indirect realization of $\mathrm{C}_{33}$ (type $\mathrm{I}$ nonsingular): Vanishing of $M_{\nu 11}+M_{\nu 22}$}

We saw that the matrix $S_{33}$ conjures, when acted on $M_{\nu}$, the combination $M_{\nu 22}+M_{\nu 33}$ in the element $(1,2)$ of the transformed $\tilde{M}_{\nu}$. Thus we follow [5] and impose $Z_{8} \times Z_{2}$ symmetry to have a zero in the $(1,2)$ entry of the unrotated $M_{\nu 0}$ and check that the rotated mass matrix $M_{\nu}=S^{T} M_{\nu 0} S$ has a texture $\mathbf{C}_{33}$ with $S=S_{33}$,

$$
\begin{aligned}
S & =\frac{1}{\sqrt{2}}\left(\begin{array}{ccc}
i & -1 & 0 \\
i & 1 & 0 \\
0 & 0 & \sqrt{2}
\end{array}\right) \Rightarrow S^{T}\left(\begin{array}{ccc}
A & 0 & C \\
0 & D & E \\
C & E & F
\end{array}\right) S \\
& =\left(\begin{array}{ccc}
-\frac{1}{2}(A+D) & \frac{i}{2}(D-A) & \frac{i}{\sqrt{2}}(E+C) \\
\frac{i}{2}(D-A) & \left.\frac{1}{2}(A+D)\right) & \frac{1}{\sqrt{2}}(E-C) \\
\frac{i}{\sqrt{2}}(E+C) & \frac{1}{\sqrt{2}}(E-C) & F
\end{array}\right) .
\end{aligned}
$$

First, we show how one can impose the zero texture. We introduce five Standard Model (SM) Higgs doublets $\Phi_{a}(a=1, \ldots, 5)$, use three real scalar singlets $\chi_{i}(i=1,2$, 3 ), and denote the left-handed lepton doublet of the first (second, third) family by $D_{L 1}\left(D_{L 2}, D_{L 3}\right)$. The right-handed charged lepton and neutrino singlets are denoted by $\left(\ell_{R}\right.$, $\left.\nu_{R}\right)$. We assume the transformations shown in Table IV under $Z_{8}^{0} \times Z_{2}^{0}$ for the fields.

By forming bilinear terms of $\bar{D}_{L i} \ell_{R j}$ and $\bar{D}_{L i} \nu_{R j}$, relevant for Dirac mass matrices of neutrino and charged leptons, and of $\nu_{R i} \nu_{R j}$, relevant for the Majorana neutrino mass matrix $M_{R}$ in the Lagrangian $\left[Y_{i j}^{a}\right.$ are the Yaukawa coupling constants, the indices $(i, j)$ are flavor ones, the indices $(a, b)$ run, respectively, over the Higgs doublet and scalar singlet fields, $\mathcal{C}$ is the charge conjugation matrix, and $\left.\tilde{\Phi}=i \sigma_{2} \Phi^{*}\right]$,

$$
\begin{aligned}
\mathcal{L}_{M} \supset & \sum_{i, j=1}^{3} \sum_{a=1}^{5} \sum_{b=1}^{3} Y_{0 \chi i j}^{b} \chi_{b} \nu_{R i}^{T} \mathcal{C}^{-1} \nu_{R j}+Y_{0 D i j}^{a} \bar{D}_{L i} \tilde{\Phi}_{a} \nu_{R j} \\
& +Y_{0 \ell i j}^{a} \bar{D}_{L i} \Phi_{a} \ell_{R j}
\end{aligned}
$$

TABLE IV. The $Z_{8}^{0} \times Z_{2}^{0}$ symmetry realization of the one zero texture at the $(1,2)$ entry corresponding upon rotation to vanishing subtrace $\mathbf{C}_{33}$. The index $D_{L 1}$ indicates the left-handed lepton doublet first family and so on. The $\chi_{k}$ denotes a scalar singlet that produces an entry in the right-handed Majorana mass matrix when acquiring a VEV at the seesaw scale. $\omega$ denotes $e^{i \pi / 4}$.

Symmetry under $Z_{8}^{0}$ factor

$\begin{array}{lllllllllllllll}\Phi_{1} & \Phi_{2} & \Phi_{3} & \Phi_{4} & \Phi_{5} & D_{L 1} & D_{L 2} & D_{L 3} & \nu_{R 1} & \nu_{R 2} & \nu_{R 3} & \chi_{1} & \chi_{2} & \chi_{3} & \ell_{R}\end{array}$ $\begin{array}{lllllllllllllll}1 & \omega^{4} & \omega & 1 & \omega^{7} & 1 & \omega^{4} & \omega & 1 & \omega^{4} & \omega & 1 & \omega^{3} & \omega^{6} & 1\end{array}$ Symmetry under $Z_{2}^{0}$ factor \begin{tabular}{lllllllllllllll}
1 & 1 & 1 & $\omega^{4}$ & $\omega^{4}$ & 1 & 1 & 1 & $\omega^{4}$ & $\omega^{4}$ & $\omega^{4}$ & 1 & 1 & 1 & 1 \\
\hline \hline
\end{tabular} and examining how they transform under $Z_{8}^{0} \times Z_{2}^{0}$, we see that the invariance under the symmetry implies the following forms:

$$
\begin{aligned}
M_{D 0} & =\left(\begin{array}{ccc}
\times & 0 & 0 \\
0 & \times & 0 \\
\times & 0 & \times
\end{array}\right), \quad M_{R 0}=\left(\begin{array}{ccc}
\times & 0 & 0 \\
0 & \times & \times \\
0 & \times & \times
\end{array}\right) \\
\Rightarrow M_{\nu 0} & =M_{D 0} M_{R 0}^{-1} M_{D 0}^{T}=\left(\begin{array}{ccc}
\times & 0 & \times \\
0 & \times & \times \\
\times & \times & \times
\end{array}\right) .
\end{aligned}
$$

Note that, in contrast to [5] where we introduced only three Higgs doublets, we introduce here five Higgs doublets; otherwise, we would have got as in [5] a diagonal charged lepton mass matrix before proceeding to the rotation defined by $S$ of Eq. (57). Had we done this then, we should have gotten field transformations corresponding to the rotated symmetry by adjoint acting on the unrotated transformations by the rotation $S$, which will produce a nondiagonal matrix for the charged leptons, which means that upon rotating and getting the vanishing subtrace texture we would have left the flavor basis. Actually, we added the extra Higgs fields exactly in order to get a generic charged lepton mass matrix in the unrotated basis while keeping the form of the Dirac neutrino mass matrix. The fields $\Phi_{4,5}$ are responsible for the desired form of $M_{D 0}$, whereas the fields $\left(\Phi_{1,2,3}\right)$ produce generic $M_{\ell 0}$.

In order to find the new rotated symmetry, we need to find then how all the fields would transform. Thus, we should explicitly write down the form of the mass matrices in terms of the Yukawa couplings when the Higgs and singlet scalar fields get VEVs. Actually the invariance of the Majorana term under $Z_{8}^{0} \times Z_{2}^{0}$ implies the following constraint:

$$
\left(Y_{0 \chi}^{b}\right)=T_{\chi a b}^{0 Z}\left(T_{\nu R}^{0 Z}\right)^{T}\left(Y_{0 \chi}^{a}\right)\left(T_{\nu_{R}}^{0 Z}\right),
$$

where $(a, b=1,2,3),\left(Y_{0 \chi}^{b}\right)$ is a matrix in flavor space with element $Y_{0 \chi i j}^{b}$ at its $(i, j)$ th entry, and $T_{f}^{0 Z}\left(f=\chi, \nu_{R}\right)$ is a matrix (diagonal by construction) defining the transformation of the field $f$ under the considered symmetry factor $Z$ $\left(Z=Z_{8}^{0}\right.$ or $Z_{2}^{0}$ ). This constraint [Eq. (60)] can be solved for both symmetry factors and leads to the following form, when $\chi_{a}$ gets a VEV $v_{0 \chi_{a}}$ :

$$
M_{R 0}=\left(\begin{array}{ccc}
Y_{0 \chi 11}^{1} v_{0 \chi_{1}} & 0 & 0 \\
0 & Y_{0 \chi 22}^{1} v_{0 \chi_{1}} & Y_{0 \chi 23}^{2} v_{0 \chi_{2}} \\
0 & Y_{0 \chi 23}^{2} v_{0 \chi_{2}} & Y_{0 \times 33}^{3} v_{0 \chi_{3}}
\end{array}\right) \text {. }
$$

The invariance of the Dirac neutrino mass term under $Z_{8}^{0} \times Z_{2}^{0}$ implies the following constraint: 


$$
\left(Y_{0 D}^{b}\right)=\left(T_{\Phi}^{0 Z}\right)_{a b}^{\dagger}\left(T_{D_{L}}^{0 Z}\right)^{\dagger}\left(Y_{0 D}^{a}\right)\left(T_{\nu_{R}}^{0 Z}\right),
$$

where $(a, b=1, \ldots, 5),\left(Y_{0 D}^{a}\right)$ is a matrix in flavor space with element $Y_{0 D i j}^{a}$ at its $(i, j)$ th entry, and $\left(T_{f}^{0 Z}\right)(f=\Phi$, $\left.D_{L}, \nu_{R}\right)$ is a diagonal-by construction-matrix defining the transformation of the field $f$ under the considered symmetry factor $Z\left(Z=Z_{8}^{0}\right.$ or $\left.Z_{2}^{0}\right)$ which leads when solved for both $Z_{8}^{0}$ and $Z_{2}^{0}$ to the following form, when $\Phi_{a}$ gets a VEV $v_{0 \Phi_{a}}$ :

$$
M_{D 0}=\left(\begin{array}{ccc}
Y_{0 D 11}^{4} v_{0 \Phi_{4}} & 0 & 0 \\
0 & Y_{0 D 22}^{4} v_{0 \Phi_{4}} & 0 \\
Y_{0 D 31}^{5} v_{0 \Phi_{5}} & 0 & Y_{0 D 33}^{4}{ } v_{0 \Phi_{4}}
\end{array}\right) .
$$

And we get $M_{\nu 0}=M_{D 0} M_{R 0}^{-1} M_{D 0}^{T}$ of the desired form with vanishing element at the $(2,1)$ th entry. As to the charged lepton mass matrix, the invariance of the corresponding mass term gives

$$
\left(Y_{0 \ell}^{b}\right)=T_{\Phi a b}^{0 Z}\left(T_{D_{L}}^{0 Z}\right)^{\dagger}\left(Y_{0 \ell}^{a}\right)\left(T_{\ell_{R}}^{0 Z}\right),
$$

where $(a, b=1, \ldots, 5),\left(Y_{0 \ell}^{a}\right)$ is a matrix in flavor space with element $Y_{0 \ell i j}^{a}$ at its $(i, j)$ th entry, and $T_{f}^{0 Z}(f=\Phi$, $\left.D_{L}, \ell_{R}\right)$ is a matrix defining the transformation of the field $f$ under the considered symmetry factor $Z\left(Z=Z_{8}^{0}\right.$ or $\left.Z_{2}^{0}\right)$ which leads to a generic form for the charged lepton matrix:

$$
M_{\ell 0}=\left(\begin{array}{lll}
Y_{0 \ell 11}^{1} v_{0 \Phi_{1}} & Y_{0 \ell 12}^{1} v_{0 \Phi_{1}} & Y_{0 \ell 13}^{1} v_{0 \Phi_{1}} \\
Y_{0 \ell 21}^{2} v_{0 \Phi_{2}} & Y_{0 \ell 22}^{2} v_{0 \Phi_{2}} & Y_{0 \ell 23}^{2} v_{0 \Phi_{2}} \\
Y_{0 \ell 31}^{3} v_{0 \Phi_{3}} & Y_{0 \ell 32}^{3} v_{0 \Phi_{3}} & Y_{0 \ell 33}^{3} v_{0 \Phi_{3}}
\end{array}\right)
$$

In order to find the field transformations corresponding to the new rotated symmetry defined by $S$ [Eq. (57)], we apply the same rule as in Eq. (46) or Eq. (52), with caution, for all the fields $f$,

$$
T_{f}^{Z}=S^{\dagger} T_{f}^{0 Z} S
$$

and extending in the case of the five-dimensional $\Phi$ the matrix $S$ to be $S_{e x}=\operatorname{diag}\left(S, 1_{2 \times 2}\right)$. We state in Table $\mathrm{V}$ the resulting assignments for the fields under $Z_{8} \times Z_{2}$.

Note that we do not get generally diagonal matrices $T_{f}^{Z}$ because of the rotation $S$. Thus one can write down similar constraints to those of Eqs. (60), (62), and (64) corresponding to the rotated symmetry, albeit with Yukawa couplings and VEVs without the subscript 0 , and by solving them we get

$$
M_{R}=\left(\begin{array}{ccc}
-Y_{\chi 22}^{1}\left(v_{\chi_{1}}+i v_{\chi_{2}}\right) & -i Y_{\chi 12}^{2}\left(v_{\chi_{1}}+i v_{\chi_{2}}\right) & Y_{\chi 23}^{2}\left(-v_{\chi_{1}}+i v_{\chi_{2}}\right) \\
- & Y_{\chi 22}^{1}\left(v_{\chi_{1}}+i v_{\chi_{2}}\right) & -i Y_{\chi 23}^{2}\left(-v_{\chi_{1}}+i v_{\chi_{2}}\right) \\
- & - & Y_{\chi 33}^{3} v_{\chi_{3}}
\end{array}\right)
$$

where - denotes an element deduced by symmetry property of the matrix $\left(M=M^{T}\right)$, and this convention will be used from now on,

$$
M_{D}=\left(\begin{array}{ccc}
Y_{D 22}^{4} v_{\Phi_{4}} & -Y_{D 21}^{4} v_{\Phi_{4}} & 0 \\
Y_{D 21}^{4} v_{\Phi_{4}} & Y_{D 22}^{4} v_{\Phi_{4}} & 0 \\
-i Y_{D 32}^{5} v_{\Phi_{5}} & Y_{D 32}^{5} v_{\Phi_{5}} & Y_{D 33}^{4} v_{\Phi_{4}}
\end{array}\right)
$$

One can check that the resulting $M_{\nu}$ satisfies the texture $\mathbf{C}_{33}$. Note also that all the Yukawa couplings and the VEVs in Eqs (67) and (68) are different from those in Eqs (61) and (63) since each set of Yukawa couplings and VEVs correspond to the Lagrangian under a specific symmetry. However, they are related through the transformation

$$
M=S^{T} M_{0} S
$$

which should be valid for $\left(M_{\nu}, M_{R}, M_{D}\right)$, and one can check that the form of $M_{(\nu, D, R)}$ is the same as that of $S^{T} M_{(\nu 0, D 0, R 0)} S$.

We need to show now that the symmetry-gauge basis for the charged leptons, in which the symmetry was given, can under natural assumptions be taken to a very good approximation, to be the flavor basis. Actually, we get a generic mass matrix $M_{\ell}$ :

$$
M_{\ell}=\left(\begin{array}{ccc}
Y_{\ell 21}^{2} v_{\Phi_{1}}-Y_{\ell 21}^{1} v_{\Phi_{2}} & Y_{\ell 22}^{2} v_{\Phi_{1}}-Y_{\ell 22}^{1} v_{\Phi_{2}} & Y_{\ell 23}^{2} v_{\Phi_{1}}-Y_{\ell 23}^{1} v_{\Phi_{2}} \\
Y_{\ell 21}^{1} v_{\Phi_{1}}+Y_{\ell 21}^{2} v_{\Phi_{2}} & Y_{\ell 22}^{1} v_{\Phi_{1}}+Y_{\ell 22}^{2} v_{\Phi_{2}} & Y_{\ell 23}^{1} v_{\Phi_{1}}+Y_{\ell 23}^{2} v_{\Phi_{2}} \\
Y_{\ell 31}^{3} v_{\Phi_{3}} & Y_{\ell 32}^{3} v_{\Phi_{3}} & Y_{\ell 33}^{3} v_{\Phi_{3}}
\end{array}\right),
$$


TABLE V. The $Z_{8} \times Z_{2}$ symmetry realization of the vanishing subtrace $\mathbf{C}_{33}$. The $D_{L 1}$ indicates the left-handed lepton doublet first family and so on. The $\chi_{k}$ denotes a scalar singlet that produces an entry in the right-handed Majorana mass matrix when acquiring a VEV at the seesaw scale. The right-handed charged leptons $\ell_{R}$ are assumed singlets under the discrete symmetry. $\omega$ denotes $e^{i \pi / 4}$.

\begin{tabular}{|c|c|c|c|c|}
\hline \multicolumn{5}{|l|}{ Symmetry under $Z_{8}$ factor } \\
\hline $\boldsymbol{\Phi}=\left(\Phi_{1}, \Phi_{2}, \Phi_{3}, \Phi_{4}, \Phi_{5}\right)^{T}$ & $\boldsymbol{D}_{\boldsymbol{L}}=\left(D_{L 1}, D_{L 2}, D_{L 3}\right)^{T}$ & $\boldsymbol{\nu}_{\boldsymbol{R}}=\left(\nu_{R 1}, \nu_{R 2}, \nu_{R 3}\right)^{T}$ & $\chi=\left(\chi_{1}, \chi_{2}, \chi_{3}\right.$ & \\
\hline$\left(\begin{array}{lllll}0 & i & 0 & 0 & 0\end{array}\right)$ & $\left(\begin{array}{ll}0 & i\end{array}\right.$ & $0)$ & $\left(1+\omega^{3}\right) \quad i\left(1-\omega^{3}\right)$ & 0 \\
\hline$-i \quad 0 \quad 0 \quad 0 \quad 0 \quad 0$ & $\begin{array}{ll}-i & 0\end{array}$ & 0 & $\frac{2}{2} \quad \frac{2}{2}$ & \\
\hline $\begin{array}{lllll}0 & 0 & \omega & 0 & 0\end{array}$ & $1 \begin{array}{ll}0 & 0\end{array}$ & $\omega)$ & $\frac{i\left(-1+\omega^{3}\right)}{2} \quad \frac{\left(1+\omega^{3}\right)}{2}$ & 0 \\
\hline $\begin{array}{lllll}0 & 0 & 0 & 1 & 0\end{array}$ & & & 0 & $\omega^{6}$ \\
\hline$\left(\begin{array}{lllll}0 & 0 & 0 & 0 & \omega^{7}\end{array}\right)$ & & & & \\
\hline $\begin{array}{l}\text { Symmetry under } Z_{2} \text { factor } \\
\operatorname{diag}(1,1,1,-1,-1)\end{array}$ & $\operatorname{diag}(1,1,1)$ & $\operatorname{diag}(-1,-1,-1)$ & $\operatorname{diag}(1,1,1)$ & \\
\hline
\end{tabular}

so if assume the related VEVs are comparable, $v_{\Phi_{1}} \approx v_{\Phi_{2}} \approx$ $v_{\Phi_{3}} \approx v$, then we get

$$
\begin{aligned}
M_{\ell} & \approx v\left(\begin{array}{ccc}
Y_{\ell 21}^{2}-Y_{\ell 21}^{1} & Y_{\ell 22}^{2}-Y_{\ell 22}^{1} & Y_{\ell 23}^{2}-Y_{\ell 23}^{1} \\
Y_{\ell 21}^{1}+Y_{\ell 21}^{2} & Y_{\ell 22}^{1}+Y_{\ell 22}^{2} & Y_{\ell 23}^{1}+Y_{\ell 23}^{2} \\
Y_{\ell 31}^{3} & Y_{\ell 32}^{3} & Y_{\ell 33}^{3}
\end{array}\right) \\
& =v\left(\begin{array}{l}
\mathbf{a}^{T} \\
\mathbf{b}^{T} \\
\mathbf{c}^{T}
\end{array}\right),
\end{aligned}
$$

where $\mathbf{a}, \mathbf{b}$, and $\mathbf{c}$ stand for column vectors extracted from the corresponding rows, formed of Yukawa couplings, in the matrix $M_{\ell}$, and this abbreviation will be used from now on. The dot product refers to the usual Hermitian inner product defined as $\mathbf{a} \cdot \mathbf{b}=\sum_{i=1}^{3} a_{i} b_{i}^{*}$. Thus

$$
M_{\ell} M_{\ell}^{\dagger} \approx v^{2}\left(\begin{array}{ccc}
\mathbf{a} \cdot \mathbf{a} & \mathbf{a} \cdot \mathbf{b} & \mathbf{a} \cdot \mathbf{c} \\
\mathbf{b} \cdot \mathbf{a} & \mathbf{b} \cdot \mathbf{b} & \mathbf{b} \cdot \mathbf{c} \\
\mathbf{c} \cdot \mathbf{a} & \mathbf{c} \cdot \mathbf{b} & \mathbf{c} \cdot \mathbf{c}
\end{array}\right)
$$

so taking only the following natural assumption on the norms of the vectors:

$$
\begin{aligned}
\|\mathbf{a}\| /\|\mathbf{c}\| & =m_{e} / m_{\tau} \sim 3 \times 10^{-4} \\
\|\mathbf{b}\| /\|\mathbf{c}\| & =m_{\mu} / m_{\tau} \sim 6 \times 10^{-2}
\end{aligned}
$$

one can diagonalize $M_{\ell} M_{\ell}^{\dagger}$ by an infinitesimal rotation as was done in [5], which proves that we are to a good approximation in the flavor basis.

Some remarks are in order here. First, one would naturally assume Yukawa couplings of the same order, and the assumption || $\mathbf{a}|\ll| \mid \mathbf{b}\|\ll\| \mathbf{c} \|$ cannot be met unless there is fine-tuning in the Yukawas. We find nothing wrong with the needed fine-tuning, especially that an analogous fine-tuning, to enforce the charged lepton mass hierarchies, is needed in many similar models, and even in the SM [18]. Second, as said earlier and in line with [19], the subtrace texture is zero by construction in the symmetry-gauge basis of the neutrino fields, whereas the gauge-symmetry basis of the charged leptons is deviated infinitesimally from the flavor basis, and this deviation is of the order of the "acute" charged lepton masses' hierarchies, which means we are to a very good approximation in the flavor basis.

\section{B. Indirect realization of $\mathbf{C}_{11}$ (type I nonsingular): Vanishing of $M_{\nu 22}+M_{\nu 33}$}

Following the same procedure as for the case $\mathbf{C}_{33}$, we just state briefly the results. The rotation matrix which moves a zero texture at $(2,3)$ to the texture $\mathbf{C}_{11}$ is given by

$$
\begin{aligned}
S & =\frac{1}{\sqrt{2}}\left(\begin{array}{ccc}
\sqrt{2} & 0 & 0 \\
0 & i & -1 \\
0 & i & 1
\end{array}\right) \Rightarrow S^{T}\left(\begin{array}{ccc}
A & B & C \\
B & D & 0 \\
C & 0 & F
\end{array}\right) S \\
& =\left(\begin{array}{ccc}
A & \frac{i}{\sqrt{2}}(B+C) & -\frac{1}{\sqrt{2}}(B-C) \\
- & \left.-\frac{1}{2}(D+F)\right) & -\frac{i}{2}(D-F) \\
- & - & \frac{1}{2}(D+F)
\end{array}\right),
\end{aligned}
$$

TABLE VI. The $Z_{8}^{0} \times Z_{2}^{0}$ symmetry realization of the one zero texture at the $(2,3)$ entry corresponding upon rotation to vanishing subtrace $\mathbf{C}_{11}$. The $D_{L 1}$ indicates the left-handed lepton doublet first family and so on. The $\chi_{k}$ denotes a scalar singlet which produces an entry in the right-handed Majorana mass matrix when acquiring a VEV at the seesaw scale. The right-handed charged leptons $\ell_{R}$ are assumed singlets under the discrete symmetry. $\omega$ denotes $e^{i \pi / 4}$.

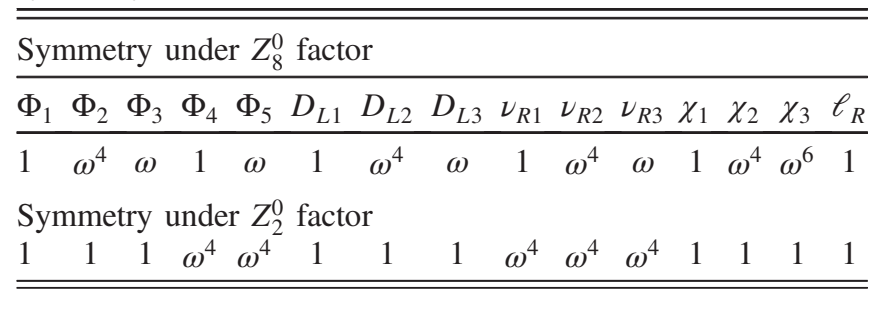


TABLE VII. The $Z_{8} \times Z_{2}$ symmetry realization of the vanishing subtrace $\mathbf{C}_{11}$. The $D_{L 1}$ indicates the left-handed lepton doublet first family and so on. The $\chi_{k}$ denotes a scalar singlet which produces an entry in the right-handed Majorana mass matrix when acquiring a VEV at the seesaw scale. The right-handed charged leptons are assumed to be singlets under the discrete symmetry. $\omega$ denotes $e^{i \pi / 4}$.

Symmetry under $Z_{8}$ factor

$\Phi=\left(\Phi_{1}, \Phi_{2}, \Phi_{3}, \Phi_{4}, \Phi_{5}\right)^{T}$

$\left(\begin{array}{ccccc}1 & 0 & 0 & 0 & 0 \\ 0 & \frac{-1+\omega}{2} & \frac{-i(1+\omega)}{2} & 0 & 0 \\ 0 & \frac{i(1+\omega)}{2} & \frac{-1+\omega}{2} & 0 & 0 \\ 0 & 0 & 0 & 1 & 0 \\ 0 & 0 & 0 & 0 & \omega\end{array}\right)$

$\boldsymbol{D}_{\boldsymbol{L}}=\left(D_{L 1}, D_{L 2}, D_{L 3}\right)^{T} \quad \boldsymbol{\nu}_{\boldsymbol{R}}=\left(\nu_{R 1}, \nu_{R 2}, \nu_{R 3}\right)^{T}$

$\left(\begin{array}{ccc}1 & 0 & 0 \\ 0 & \frac{-1+\omega}{2} & \frac{-i(1+\omega)}{2} \\ 0 & \frac{i(1+\omega)}{2} & \frac{-1+\omega}{2}\end{array}\right)$ $\left.\begin{array}{ccc}\chi=\left(\chi_{1}, \chi_{2}, \chi_{3}\right)^{T} \\ 1 & 0 & 0 \\ 0 & \frac{-1+\omega^{6}}{2} & \frac{-i\left(1+\omega^{6}\right)}{2} \\ 0 & \frac{i\left(1+\omega^{6}\right)}{2} & \frac{-1+\omega^{6}}{2}\end{array}\right)$

$\operatorname{diag}(1,1,1)$

$\operatorname{diag}(1,1,1,-1,-1)$

$\operatorname{diag}(1,1,1)$

$\operatorname{diag}(-1,-1,-1)$

transformations for the fields corresponding to the new symmetry imposing the vanishing subtrace by the rule in Eq. (66), but with $S$ as given in Eq. (74). We state in Table VII the resulting assignments for the fields under $Z_{8} \times Z_{2}$.

The rotated symmetry imposes some constraints on the Yukawa couplings and the VEVs, which when solved give the following results for $M_{R}$ and $M_{D}$ :

above transformations force a neutrino mass matrix with zero texture at the $(2,3)$ entry. Again we define the new

$$
M_{R}=\left(\begin{array}{ccc}
Y_{\chi 11}^{1} v_{\chi_{1}} & Y_{\chi 12}^{2}\left(v_{\chi_{2}}+i v_{\chi_{3}}\right) & i Y_{\chi 12}^{2}\left(v_{\chi_{2}}+i v_{\chi_{3}}\right) \\
- & i\left(-Y_{\chi 23}^{1} v_{\chi_{1}}+i Y_{\chi 33}^{2} v_{\chi_{2}}+Y_{\chi 33}^{2} v_{\chi_{3}}\right) & Y_{\chi^{23}}^{1} v_{\chi_{1}}+i Y_{\chi_{33}}^{2} v_{\chi_{2}}+Y_{\chi_{33}}^{2} v_{\chi_{3}} \\
- & - & -i\left(-Y_{\chi^{23}}^{1} v_{\chi_{1}}+i Y_{\chi_{33}}^{2} v_{\chi_{2}}+Y_{\chi_{33}}^{2} v_{\chi_{3}}\right)
\end{array}\right)
$$

and

$$
M_{D}=\left(\begin{array}{ccc}
Y_{D 11}^{4} v_{\Phi_{4}} & i Y_{D 13}^{5} v_{\Phi_{5}} & Y_{D 13}^{5} v_{\Phi_{5}} \\
0 & Y_{D 22}^{4} v_{\Phi_{4}} & Y_{D 23}^{4} v_{\Phi_{4}} \\
0 & -Y_{D 23}^{4} v_{\Phi_{4}} & Y_{D 22}^{4} v_{\Phi_{4}}
\end{array}\right)
$$

One can check that the resulting $M_{\nu}$ satisfies the texture $\mathbf{C}_{11}$.

As to $M_{\ell}$ we get

$$
M_{\ell}=\left(\begin{array}{ccc}
Y_{\ell 11}^{1} v_{\Phi_{1}} & Y_{\ell 12}^{1} v_{\Phi_{1}} & Y_{\ell 13}^{1} v_{\Phi_{1}} \\
Y_{\ell 13}^{3} v_{\Phi_{2}}+Y_{\ell 21}^{3} v_{\Phi_{3}} & Y_{\ell 22}^{2} v_{\Phi_{2}}-Y_{\ell 32}^{2} v_{\Phi_{3}} & Y_{\ell 33}^{3} v_{\Phi_{2}}+Y_{\ell 23}^{3} v_{\Phi_{3}} \\
-Y_{\ell 21}^{3} v_{\Phi_{2}}+Y_{\ell 31}^{3} v_{\Phi_{3}} & Y_{\ell 32}^{2} v_{\Phi_{2}}+Y_{\ell 22}^{2} v_{\Phi_{3}} & -Y_{\ell 23}^{3} v_{\Phi_{2}}+Y_{\ell 33}^{3} v_{\Phi_{3}}
\end{array}\right),
$$

then we see that if we assume all the related VEVs are comparable $v_{\Phi_{1}} \approx v_{\Phi_{2}} \approx v_{\Phi_{3}} \approx v$, then we get

$$
M_{\ell} \approx v\left(\begin{array}{ccc}
Y_{\ell 11}^{1} & Y_{\ell 12}^{1} & Y_{\ell 13}^{1} \\
Y_{\ell 13}^{3}+Y_{\ell 21}^{3} & Y_{\ell 22}^{2}-Y_{\ell 32}^{2} & Y_{\ell 33}^{3}+Y_{\ell 23}^{3} \\
-Y_{\ell 21}^{3}+Y_{\ell 31}^{3} & Y_{\ell 32}^{2}+Y_{\ell 22}^{2} & -Y_{\ell 23}^{3}+Y_{\ell 33}^{3}
\end{array}\right)=v\left(\begin{array}{c}
\mathbf{a}^{T} \\
\mathbf{b}^{T} \\
\mathbf{c}^{T}
\end{array}\right)
$$

which can be diagonalized by an infinitesimal rotation under some natural assumptions on the amplitudes of the Yukawa vectors as done for the case of $\mathbf{C}_{33}$. 


\section{Indirect realization of $\mathbf{C}_{22}$ (type $I$ nonsingular): Vanishing of $M_{\nu 11}+M_{\nu 33}$}

The rotation matrix which moves a zero texture at $(1,3)$ to the texture $\mathbf{C}_{22}$ is given by

$$
\begin{aligned}
S & =\frac{1}{\sqrt{2}}\left(\begin{array}{ccc}
i & 0 & -1 \\
0 & \sqrt{2} & 0 \\
i & 0 & 1
\end{array}\right) \Rightarrow S^{T}\left(\begin{array}{ccc}
A & B & 0 \\
B & D & E \\
0 & E & F
\end{array}\right) S \\
& =\left(\begin{array}{ccc}
-\frac{1}{2}(A+F) & \frac{i}{\sqrt{2}}(B+E) & \frac{i}{\sqrt{2}}(F-A) \\
- & D & \frac{1}{\sqrt{2}}(E-B) \\
- & - & \frac{1}{2}(A+F)
\end{array}\right),
\end{aligned}
$$

and we check that the sum of elements at $(1,1)$ and $(3,3)$ vanishes.

At the Lagrangian level, the symmetry transformations for the fields which impose a zero texture $M_{\nu 0}$ with generic $M_{\ell 0}$ are given in Table VIII.

By forming bilinear terms of the fields we see that the above transformations force the $(1,3)$ entry in $M_{\nu 0}$ to vanish. Again we define the new transformations for the
TABLE VIII. The $Z_{8}^{0} \times Z_{2}^{0}$ symmetry realization of the one zero texture at the $(1,3)$ entry corresponding upon rotation to vanishing subtrace $\mathbf{C}_{22}$. The $D_{L 1}$ indicates the left-handed lepton doublet first family and so on. The $\chi_{k}$ denotes a scalar singlet which produces an entry in the right-handed Majorana mass matrix when acquiring a VEV at the seesaw scale. $\omega$ denotes $e^{i \pi / 4}$.

Symmetry under $Z_{8}^{0}$ factor

$\begin{array}{lllllllllllllll}\Phi_{1} & \Phi_{2} & \Phi_{3} & \Phi_{4} & \Phi_{5} & D_{L 1} & D_{L 2} & D_{L 3} & \nu_{R 1} & \nu_{R 2} & \nu_{R 3} & \chi_{1} & \chi_{2} & \chi_{3} & \ell_{R}\end{array}$ $\begin{array}{lllllllllllllll}1 & \omega^{4} & \omega & 1 & \omega^{5} & \omega & \omega^{4} & 1 & \omega & \omega^{4} & 1 & 1 & \omega^{6} & \omega^{6} & 1\end{array}$

Symmetry under $Z_{2}^{0}$ factor

\begin{tabular}{lllllllllllllll}
1 & 1 & 1 & $\omega^{4}$ & $\omega^{4}$ & 1 & 1 & 1 & $\omega^{4}$ & $\omega^{4}$ & $\omega^{4}$ & 1 & 1 & 1 & 1 \\
\hline \hline
\end{tabular}

fields corresponding to the new symmetry imposing the vanishing subtrace by the rule in Eq. (66), but with $S$ given by Eq. (79).

We state in Table IX the resulting assignments for the fields under $Z_{8} \times Z_{2}$.

The rotated symmetry imposes some constraints on the Yukawa couplings and the VEVs, which when solved give the following results:

$$
M_{R}=\left(\begin{array}{ccc}
i\left[Y_{\chi 33}^{3}\left(v_{\chi_{1}}+i v_{\chi_{3}}\right)-Y_{\chi 13}^{2} v_{\chi_{2}}\right] & Y_{\chi 23}^{3}\left(-v_{\chi_{1}}+i v_{\chi_{3}}\right) & Y_{\chi^{33}}^{3}\left(v_{\chi_{1}}+i v_{\chi_{3}}\right)+Y_{\chi 13}^{2} v_{\chi_{2}} \\
- & -i Y_{\chi 22}^{3}\left(v_{\chi_{1}}+i v_{\chi_{3}}\right) & -i Y_{\chi^{23}}^{3}\left(-v_{\chi_{1}}+i v_{\chi_{3}}\right) \\
- & - & -i\left[Y_{\chi_{33}}^{3}\left(v_{\chi_{1}}+i v_{\chi_{3}}\right)-Y_{\chi_{13}}^{2} v_{\chi_{2}}\right]
\end{array}\right)
$$

and

$$
M_{D}=\left(\begin{array}{ccc}
Y_{D 11}^{4} v_{\Phi_{4}} & 0 & -Y_{D 31}^{4} v_{\Phi_{4}} \\
-i Y_{D 23}^{5} v_{\Phi_{5}} & Y_{D 22}^{4} v_{\Phi_{4}} & Y_{D 23}^{5} v_{\Phi_{5}} \\
Y_{D 31}^{4} v_{\Phi_{4}} & 0 & Y_{D 11}^{4} v_{\Phi_{4}}
\end{array}\right) .
$$

One can check that the resulting $M_{\nu}$ satisfies the texture $\mathbf{C}_{22}$. As to $M_{\ell}$ one gets

TABLE IX. The $Z_{8} \times Z_{2}$ symmetry realization of the vanishing subtrace $\mathbf{C}_{22}$. The $D_{L 1}$ indicates the left-handed

\begin{tabular}{|c|c|c|c|c|c|c|}
\hline \multicolumn{7}{|c|}{ Symmetry under $Z_{8}$ factor } \\
\hline \multicolumn{4}{|c|}{$\overline{\boldsymbol{\Phi}=\left(\Phi_{1}, \Phi_{2}, \Phi_{3}, \Phi_{4}, \Phi_{5}\right)^{T}}$} & $\boldsymbol{D}_{L}=\left(D_{L 1}, D_{L 2}, D_{L 3}\right)^{T}$ & $\nu_{\boldsymbol{R}}=\left(\nu_{R 1}, \nu_{R 2}, \nu_{R 3}\right)^{T}$ & $\chi=\left(\chi_{1}, \chi_{2}, \chi_{3}\right)^{T}$ \\
\hline$\frac{1+\omega}{2}$ & 0 & $\frac{i(1-\omega)}{2}$ & $0 \quad 0)$ & $\left(\begin{array}{cc}\frac{1+\omega}{2} & 0\end{array}\right.$ & $\underline{-i(1-\omega)}$ & $\left(\begin{array}{lll}0 & 0 & i\end{array}\right)$ \\
\hline $0^{2}$ & -1 & 0 & $0 \quad 0$ & $\stackrel{2}{2}_{0}^{2}$ & $\stackrel{2}{0}$ & $\begin{array}{lll}0 & \omega^{6} & 0\end{array}$ \\
\hline$\frac{-i(1-\omega)}{2}$ & 0 & $\frac{1+\omega}{2}$ & $\begin{array}{ll}0 & 0\end{array}$ & $\frac{i(1-\omega)}{2} \quad 0$ & $\frac{1+\omega}{2}$ & $-i \quad 0 \quad 0$ \\
\hline 0 & 0 & 0 & 10 & & & \\
\hline 0 & 0 & 0 & $\begin{array}{ll}0 & \omega^{5}\end{array}$ & & & \\
\hline \multicolumn{4}{|c|}{$\begin{array}{l}\text { Symmetry under } Z_{2} \text { factor } \\
\operatorname{diag}(1,1,1,-1,-1)\end{array}$} & $\operatorname{diag}(1,1,1)$ & $\operatorname{diag}(-1,-1,-1)$ & $\operatorname{diag}(1,1,1)$ \\
\hline
\end{tabular}
lepton doublet first family and so on. The $\chi_{k}$ denotes a scalar singlet which produces an entry in the right-handed Majorana mass matrix when acquiring a VEV at the seesaw scale. The right-handed charged leptons are assumed to be singlets under the discrete symmetry. $\omega$ denotes $e^{i \pi / 4}$. 


$$
M_{\ell}=\left(\begin{array}{ccc}
Y_{\ell 11}^{1} v_{\Phi_{1}}+Y_{\ell 11}^{3} v_{\Phi_{3}} & Y_{\ell 12}^{1} v_{\Phi_{1}}+Y_{\ell 12}^{3} v_{\Phi_{3}} & Y_{\ell 13}^{1} v_{\Phi_{1}}+Y_{\ell 13}^{3} v_{\Phi_{3}} \\
Y_{\ell 21}^{2} v_{\Phi_{2}} & Y_{\ell 22}^{2} v_{\Phi_{2}} & Y_{\ell 23}^{2} v_{\Phi_{2}} \\
Y_{\ell 11}^{3} v_{\Phi_{1}}-Y_{\ell 11}^{1} v_{\Phi_{3}} & Y_{\ell 12}^{3} v_{\Phi_{1}}-Y_{\ell 12}^{1} v_{\Phi_{3}} & Y_{\ell 13}^{3} v_{\Phi_{1}}-Y_{\ell 13}^{1} v_{\Phi_{3}}
\end{array}\right) ;
$$

then we see that if we assume all the related VEVs are comparable $v_{\Phi_{1}} \approx v_{\Phi_{2}} \approx v_{\Phi_{3}} \approx v$, then we get

$$
M_{\ell} \approx v\left(\begin{array}{ccc}
Y_{\ell 11}^{1}+Y_{\ell 11}^{3} & Y_{\ell 12}^{1}+Y_{\ell 12}^{3} & Y_{\ell 13}^{1}+Y_{\ell 13}^{3} \\
Y_{\ell 21}^{2} & Y_{\ell 22}^{2} & Y_{\ell 23}^{2} \\
Y_{\ell 11}^{3}-Y_{\ell 11}^{1} & Y_{\ell 12}^{3}-Y_{\ell 12}^{1} & Y_{\ell 13}^{3}-Y_{\ell 13}^{1}
\end{array}\right)=v\left(\begin{array}{c}
\mathbf{a}^{T} \\
\mathbf{b}^{T} \\
\mathbf{c}^{T}
\end{array}\right)
$$

which can be diagonalized by an infinitesimal rotation under some natural assumptions on the amplitudes of the Yukawa vectors as done for the previous two cases.

\section{Indirect realization of $\mathrm{C}_{31}$ (type $\mathrm{I}$ nonsingular): Vanishing of $M_{\nu 12}+M_{\nu 23}$}

The rotation matrix which moves a zero texture at $(2,3)$ to the texture $\mathbf{C}_{23}$ is given by

$$
\begin{aligned}
S & =\frac{1}{\sqrt{2}}\left(\begin{array}{ccc}
1 & 0 & -1 \\
0 & \sqrt{2} & 0 \\
1 & 0 & 1
\end{array}\right) \Rightarrow S^{T}\left(\begin{array}{ccc}
A & B & C \\
B & D & 0 \\
C & 0 & F
\end{array}\right) S \\
& =\left(\begin{array}{ccc}
\frac{1}{2}(A+F)+C & \frac{1}{\sqrt{2}} B & \frac{1}{\sqrt{2}}(F-A) \\
- & D & -\frac{1}{\sqrt{2}} B \\
- & - & \frac{1}{2}(A+F)-C
\end{array}\right),
\end{aligned}
$$

and we check that the sum of elements at $(1,2)$ and $(2,3)$ vanishes.
At the Lagrangian level, the symmetry transformations for the fields which impose a zero texture $M_{\nu 0}$ at the $(2,3)$ entry with generic $M_{\ell 0}$ are given in Table X.

Again we define the new transformations for the fields corresponding to the new symmetry imposing the vanishing subtrace by the adjoint action rule [Eq. (66)], but with $S$ given by Eq. (84). We state in Table XI the resulting assignments for the fields under $Z_{8} \times Z_{2}$.

We repeat that if we could guess the "nondiagonal" transformations under $Z_{8} \times Z_{2}$ of Tables V, VII, IX, and XI, then we would not have needed to resort to the rotation method relating them to simpler ones under $Z_{8}^{0} \times Z_{2}^{0}$. However, as is clear from the tables, the transformations of the Higgs and scalar fields are in particular difficult to guess directly.

The rotated symmetry imposes some constraints on the Yukawa couplings and the VEVs, which when solved give the following results:

$$
M_{R}=\left(\begin{array}{ccc}
Y_{\chi 33}^{1} v_{\chi_{1}}+Y_{\chi 13}^{1} v_{\chi_{3}} & -Y_{\chi 23}^{2} v_{\chi_{2}} & Y_{\chi 13}^{1} v_{\chi_{1}}+Y_{\chi 33}^{1} v_{\chi_{3}} \\
- & -Y_{\chi 22}^{3}\left(v_{\chi_{1}}-v_{\chi_{3}}\right) & Y_{\chi 23}^{2} v_{\chi_{2}} \\
- & - & Y_{\chi 33}^{1} v_{\chi_{1}}+Y_{\chi 13}^{1} v_{\chi_{3}}
\end{array}\right)
$$

and

$$
M_{D}=\left(\begin{array}{ccc}
Y_{D 11}^{4} v_{\Phi_{4}}+Y_{D 11}^{5} v_{\Phi_{5}} & 0 & Y_{D 31}^{4} v_{\Phi_{4}}+Y_{D 11}^{5} v_{\Phi_{5}} \\
0 & Y_{D 22}^{4} v_{\Phi_{4}} & 0 \\
-Y_{D 11}^{5} v_{\Phi_{5}}+Y_{D 31}^{4} v_{\Phi_{4}} & 0 & Y_{D 11}^{4} v_{\Phi_{4}}-Y_{D 11}^{5} v_{\Phi_{5}}
\end{array}\right)
$$

One can check that the resulting $M_{\nu}$ satisfies the texture $C_{31}$. As to $M_{\ell}$, we get

$$
M_{\ell}=\left(\begin{array}{ccc}
Y_{\ell 11}^{1} v_{\Phi_{1}}+Y_{\ell 31}^{1} v_{\Phi_{3}} & Y_{\ell 12}^{1} v_{\Phi_{1}}+Y_{\ell 32}^{1} v_{\Phi_{3}} & Y_{\ell 13}^{1} v_{\Phi_{1}}+Y_{\ell 33}^{1} v_{\Phi_{3}} \\
Y_{\ell 21}^{2} v_{\Phi_{2}} & Y_{\ell 22}^{2} v_{\Phi_{2}} & Y_{\ell 23}^{2} v_{\Phi_{2}} \\
Y_{\ell 31}^{1} v_{\Phi_{1}}+Y_{\ell 11}^{1} v_{\Phi_{3}} & Y_{\ell 32}^{1} v_{\Phi_{1}}+Y_{\ell 12}^{1} v_{\Phi_{3}} & Y_{\ell 33}^{1} v_{\Phi_{1}}+Y_{\ell 13}^{1} v_{\Phi_{3}}
\end{array}\right) ;
$$


TABLE $X$. The $Z_{8}^{0} \times Z_{2}^{0}$ symmetry realization of the one zero texture at the $(2,3)$ entry corresponding upon rotation to vanishing subtrace $\mathbf{C}_{31}$. The $D_{L 1}$ indicates the left-handed lepton doublet first family and so on. The $\chi_{k}$ denotes a scalar singlet which produces an entry in the right-handed Majorana mass matrix when acquiring a VEV at the seesaw scale. $\omega$ denotes $e^{i \pi / 4}$.

Symmetry under $Z_{8}^{0}$ factor

$\begin{array}{lllllllllllllll}\Phi_{1} & \Phi_{2} & \Phi_{3} & \Phi_{4} & \Phi_{5} & D_{L 1} & D_{L 2} & D_{L 3} & \nu_{R 1} & \nu_{R 2} & \nu_{R 3} & \chi_{1} & \chi_{2} & \chi_{3} & \ell_{R}\end{array}$

$\begin{array}{lllllllllllllll}1 & \omega^{4} & \omega & 1 & \omega & 1 & \omega^{4} & \omega & 1 & \omega^{4} & \omega & 1 & \omega^{4} & \omega^{6} & 1\end{array}$

Symmetry under $Z_{2}^{0}$ factor

\begin{tabular}{lllllllllllllll}
1 & 1 & 1 & $\omega^{4}$ & $\omega^{4}$ & 1 & 1 & 1 & $\omega^{4}$ & $\omega^{4}$ & $\omega^{4}$ & 1 & 1 & 1 & 1 \\
\hline \hline
\end{tabular}

then we see that if we assume $v \approx v_{\Phi_{1}} \approx v_{\Phi_{2}} \gg v_{\Phi_{3}}$, then we get

$$
M_{\ell} \approx v\left(\begin{array}{ccc}
Y_{\ell 11}^{1} & Y_{\ell 12}^{1} & Y_{\ell 13}^{1} \\
Y_{\ell 21}^{2} & Y_{\ell 22}^{2} & Y_{\ell 23}^{2} \\
Y_{\ell 31}^{1} & Y_{\ell 32}^{1} & Y_{\ell 33}^{1}
\end{array}\right)=v\left(\begin{array}{c}
\mathbf{a}^{T} \\
\mathbf{b}^{T} \\
\mathbf{c}^{T}
\end{array}\right)
$$

which can be diagonalized by an infinitesimal rotation under some natural assumptions on the amplitudes of the vectors as done in the previous cases.

\section{INDIRECT REALIZATION OF TYPE I SEESAW WITH $Z_{12} \times Z_{2}$ SYMMETRY FOR SINGULAR TEXTURES}

We shall adopt the same strategy of moving from the symmetry imposing a zero texture where $M_{D}$ is singular to the symmetry imposing a vanishing subtrace with again $M_{D}$ singular, which gives via seesaw type I a singular neutrino mass matrix. Again, we follow [5] to find the symmetry transformations leading to zero elements at singular $M_{\nu}$, but will add in new fields so that to get a generic charged lepton mass matrix and not a diagonal one as was the case in [5], in such a way that the new rotated symmetry, as defined in Eq. (66), leads to vanishing subtraces at singular $M_{\nu}$ and to another generic $M_{\ell}$.
The latter under some reasonable assumptions can be diagonalized via infinitesimal rotations, which put us to a good approximation in the flavor basis.

\section{A. Indirect realization of $\mathrm{C}_{33}$ (type $\mathrm{I}$ singular): Vanishing of $M_{\nu 11}+M_{\nu 22}$}

As in the nonsingular cases, we move from zero texture at $(1,2)$ to the texture $C_{33}$ by $S$ of Eq. $(57)$.

At the Lagrangian level, the symmetry transformations for the fields which impose a zero texture neutrino mass matrix with generic charged lepton mass matrix and singular Dirac neutrino mass matrix are given in Table XII.

By forming bilinear terms of the fields we see that the above transformations force a neutrino mass matrix with zero texture at the $(1,2)$ entry. Actually, we get

$$
\begin{aligned}
M_{R 0} & =\left(\begin{array}{ccc}
Y_{0 \chi 11}^{1} v_{0 \chi_{1}} & 0 & 0 \\
0 & Y_{0 \times 22}^{2} v_{0 \chi_{2}} & 0 \\
0 & 0 & Y_{0 \times 33}^{3} v_{0 \chi_{3}}
\end{array}\right), \\
M_{D 0} & =\left(\begin{array}{ccc}
Y_{0 D 11}^{4} v_{0 \Phi_{4}} & 0 & 0 \\
0 & 0 & Y_{0 D 23}^{5} v_{0 \Phi_{5}} \\
Y_{0 D 31}^{6} v_{0 \Phi_{6}} & 0 & Y_{0 D 33}^{7} v_{0 \Phi_{7}}
\end{array}\right) .
\end{aligned}
$$

We see that $M_{D 0}$ is singular, and $M_{\nu 0}=M_{D 0} M_{R 0}^{-1} M_{D 0}^{T}$ is singular with the desired form of a vanishing element at the $(1,2)$ th entry. We can check that $M_{\ell 0}$ is of generic form as the one presented in Eq. (65).

Again, in order to find the field transformations corresponding to the new rotated symmetry defined by $S$ [Eq. (57)], we apply the rule in Eq. (66) for all the fields $f$ and extending in the case of the seven-dimensional $\Phi$ the matrix $S$ to be $S_{e x}=\operatorname{diag}\left(S, 1_{4 \times 4}\right)$, in such a way that we do not get generally diagonal matrices $T_{f}^{Z}$ because of the rotation $S$. As in nonsingular cases, one can write down constraints involving the Yukawa couplings and VEVs (now without the subscript 0 ), and by solving them we get

TABLE XI. The $Z_{8} \times Z_{2}$ symmetry realization of the vanishing subtrace $\mathbf{C}_{31}$. The $D_{L 1}$ indicates the left-handed lepton doublet first family and so on. The $\chi_{k}$ denotes a scalar singlet which produces an entry in the right-handed Majorana mass matrix when acquiring a VEV at the seesaw scale. The right-handed charged leptons are assumed to be singlets under the discrete symmetry. $\omega$ denotes $e^{i \pi / 4}$.

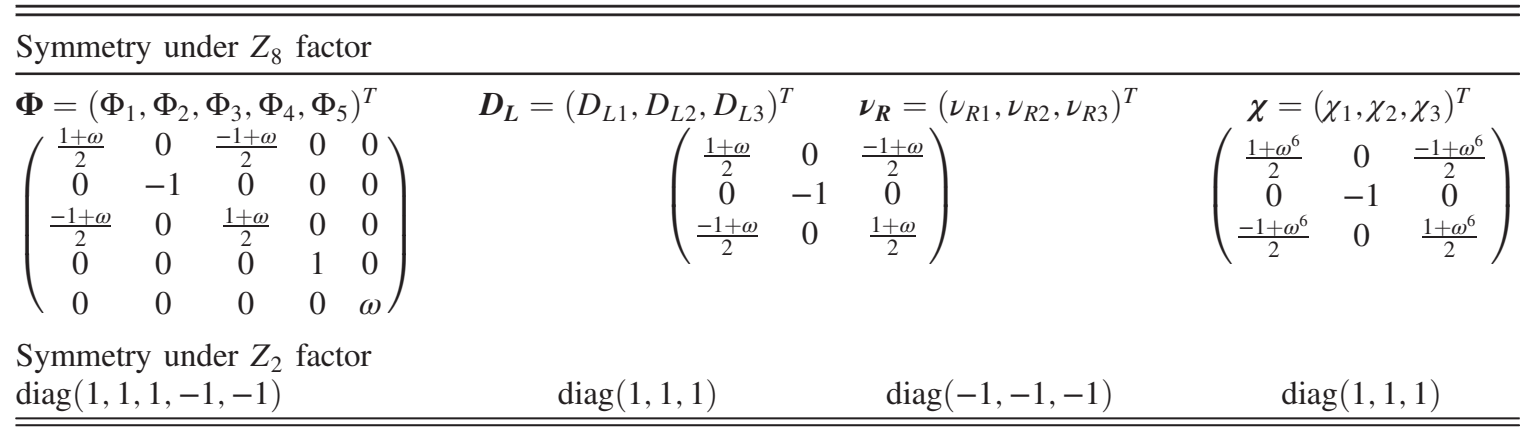


TABLE XII. The $Z_{12}^{0} \times Z_{2}^{0}$ symmetry realization of the one zero singular texture at the $(1,2)$ entry corresponding upon rotation to singular vanishing subtrace $C_{33}$. The $D_{L 1}$ indicates the lefthanded lepton doublet first family and so on. The $\chi_{k}$ denotes a scalar singlet which produces an entry in the right-handed Majorana mass matrix when acquiring a VEV at the seesaw scale. $\theta$ denotes $e^{i \pi / 6}$.

Symmetry under $Z_{12}^{0}$ factor

$\Phi_{1} \Phi_{2} \Phi_{3} \Phi_{4} \Phi_{5} \Phi_{6} \Phi_{7} D_{L 1} D_{L 2} D_{L 3} \nu_{R 1} \nu_{R 2} \nu_{R 3} \chi_{1} \chi_{2} \chi_{3} \ell_{R}$

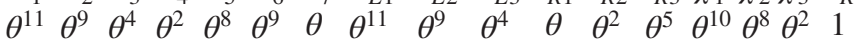

Symmetry under $Z_{2}^{0}$ factor

$\begin{array}{lllllllllllllllll}1 & 1 & 1 & \theta^{6} & \theta^{6} & \theta^{6} & \theta^{6} & 1 & 1 & 1 & \theta^{6} & \theta^{6} & \theta^{6} & 1 & 1 & 1 & 1\end{array}$

$$
\begin{aligned}
M_{R}= & \left(\begin{array}{ccc}
-Y_{\chi 22}^{1} v_{\chi_{1}}-Y_{\chi 22}^{2} v_{\chi_{2}} & -Y_{\chi 22}^{2} v_{\chi_{1}}+Y_{\chi^{22}}^{1} v_{\chi_{2}} & 0 \\
-Y_{\chi 22}^{2} v_{\chi_{1}}+Y_{\chi 22}^{1} v_{\chi_{2}} & Y_{\chi^{22}}^{1} v_{\chi_{1}}+Y_{\chi^{22}}^{2} v_{\chi_{2}} & 0 \\
0 & 0 & Y_{\chi^{33}}^{3} v_{\chi_{3}}
\end{array}\right), \\
M_{D}= & \left(\begin{array}{ccc}
-i Y_{D 12}^{4} v_{\Phi_{4}} & Y_{D 12}^{4} v_{\Phi_{4}} & -i Y_{D 23}^{5} v_{\Phi_{5}} \\
-Y_{D 12}^{4} v_{\Phi_{4}} & -i Y_{D 12}^{4} v_{\Phi_{4}} & Y_{D 23}^{5} v_{\Phi_{5}} \\
-i Y_{D 32}^{6} v_{\Phi_{6}} & Y_{D 32}^{6} v_{\Phi_{6}} & Y_{D 33}^{7} v_{\Phi_{7}}
\end{array}\right)
\end{aligned}
$$

One can check that the resulting $M_{\nu}$ is singular and satisfies the texture $\mathbf{C}_{33}$.

As to $M_{\ell}$, we get a generic mass matrix:

$$
M_{\ell}=\left(\begin{array}{ccc}
Y_{\ell 21}^{2} v_{\Phi_{1}}+Y_{\ell 11}^{2} v_{\Phi_{2}} & Y_{\ell 22}^{2} v_{\Phi_{1}}+Y_{\ell 12}^{2} v_{\Phi_{2}} & Y_{\ell 23}^{2} v_{\Phi_{1}}+Y_{\ell 13}^{2} v_{\Phi_{2}} \\
-Y_{\ell 11}^{2} v_{\Phi_{1}}+Y_{\ell 21}^{2} v_{\Phi_{2}} & -Y_{\ell 12}^{2} v_{\Phi_{1}}+Y_{\ell 22}^{2} v_{\Phi_{2}} & -Y_{\ell 13}^{2} v_{\Phi_{1}}+Y_{\ell 33}^{2} v_{\Phi_{2}} \\
Y_{\ell 31}^{3} v_{\Phi_{3}} & Y_{\ell 32}^{3} v_{\Phi_{3}} & Y_{\ell 33}^{3} v_{\Phi_{3}}
\end{array}\right) .
$$

If we assume the related VEVs are comparable $v_{\Phi_{1}} \approx v_{\Phi_{2}} \approx v_{\Phi_{3}} \approx v$, then we get

$$
M_{\ell} \approx v\left(\begin{array}{ccc}
Y_{\ell 21}^{2}+Y_{\ell 11}^{1} & Y_{\ell 22}^{2}+Y_{\ell 12}^{2} & Y_{\ell 23}^{2}+Y_{\ell 13}^{2} \\
-Y_{\ell 11}^{2}+Y_{\ell 21}^{2} & -Y_{\ell 12}^{2}+Y_{\ell 22}^{2} & -Y_{\ell 13}^{2}+Y_{\ell 23}^{2} \\
Y_{\ell 31}^{3} & Y_{\ell 32}^{3} & Y_{\ell 33}^{3}
\end{array}\right)=v\left(\begin{array}{c}
\mathbf{a}^{T} \\
\mathbf{b}^{T} \\
\mathbf{c}^{T}
\end{array}\right),
$$

whereas if we assume $v \approx v_{\Phi_{1}} \approx v_{\Phi_{3}} \gg v_{\Phi_{2}}$, we get

$M_{\ell} \approx v\left(\begin{array}{ccc}Y_{\ell 21}^{2} & Y_{\ell 22}^{2} & Y_{\ell 23}^{2} \\ -Y_{\ell 11}^{2} & -Y_{\ell 12}^{2} & -Y_{\ell 13}^{2} \\ Y_{\ell 31}^{3} & Y_{\ell 32}^{3} & Y_{\ell 33}^{3}\end{array}\right)=v\left(\begin{array}{c}\mathbf{a}^{T} \\ \mathbf{b}^{T} \\ \mathbf{c}^{T}\end{array}\right)$

In both cases, one can naturally diagonalize $M_{\ell}$ by an infinitesimal rotation, which means that we are to a good approximation in the flavor basis.

\section{B. Indirect realization of $\mathrm{C}_{11}$ (type I singular): Vanishing of $M_{\nu 22}+M_{\nu 33}$}

We move from zero texture at $(2,3)$ to the texture $\mathbf{C}_{11}$ by $S$ of Eq. (74).

The symmetry transformations for the fields which impose a zero texture $M_{\nu 0}$ with generic $M_{\ell 0}$ and singular $M_{D 0}$ are given in Table XIII. By forming bilinear terms of the fields we see that the above transformations force a neutrino mass matrix with zero texture at the $(2,3)$ entry. Again we define the new transformations for the fields corresponding to the new symmetry imposing the vanishing subtrace by the rule in Eq. (66) with $S$ given by Eq. (74) or its extension $S_{e x}$ to the seven-dimensional space of $\Phi$ 's. The rotated symmetry imposes some constraints on the
Yukawa couplings and the VEVs, which when solved give the following results:

$$
\begin{aligned}
M_{R} & =\left(\begin{array}{ccc}
Y_{\chi 11}^{1} v_{\chi_{1}} & 0 & 0 \\
0 & -Y_{\chi 23}^{3} v_{\chi_{1}}+Y_{\chi 23}^{2} v_{\chi_{3}} & Y_{\chi 23}^{2} v_{\chi_{2}}+Y_{\chi 23}^{3} v_{\chi_{3}} \\
0 & Y_{\chi 23}^{2} v_{\chi_{1}}+Y_{\chi 23}^{3} v_{\chi_{3}} & Y_{\chi 21}^{3} v_{\chi_{2}}-Y_{\chi 23}^{2} v_{\chi_{3}}
\end{array}\right), \\
M_{D} & =\left(\begin{array}{ccc}
Y_{D 11}^{4} v_{\Phi_{4}} & i Y_{D 13}^{5} v_{\Phi_{5}} & Y_{D 13}^{5} v_{\Phi_{5}} \\
i Y_{D 31}^{6} v_{\Phi_{6}} & Y_{D 33}^{7} v_{\Phi_{7}} & -i Y_{D 33}^{7} v_{\Phi_{7}} \\
Y_{D 31}^{6} v_{\Phi_{6}} & i Y_{D 33}^{7} v_{\Phi_{7}} & Y_{D 33}^{7} v_{\Phi_{7}}
\end{array}\right)
\end{aligned}
$$

TABLE XIII. The $Z_{12}^{0} \times Z_{2}^{0}$ symmetry realization of the one zero singular texture at the $(2,3)$ entry corresponding upon rotation to singular vanishing subtrace $\mathbf{C}_{11}$. The $D_{L 1}$ indicates the left-handed lepton doublet first family and so on. The $\chi_{k}$ denotes a scalar singlet which produces an entry in the right-handed Majorana mass matrix when acquiring a VEV at the seesaw scale. $\theta$ denotes $e^{i \pi / 6}$.

Symmetry under $Z_{12}^{0}$ factor

$\Phi_{1} \Phi_{2} \Phi_{3} \Phi_{4} \Phi_{5} \Phi_{6} \Phi_{7} D_{L 1} D_{L 2} D_{L 3} \nu_{R 1} \nu_{R 2} \nu_{R 3} \chi_{1} \chi_{2} \chi_{3} \ell_{R}$ $\begin{array}{lllllllllllllllll}\theta^{11} & \theta^{9} & \theta^{4} & \theta^{2} & \theta^{6} & \theta^{4} & \theta & \theta^{11} & \theta^{9} & \theta^{4} & \theta & \theta^{2} & \theta^{5} & \theta^{10} & \theta^{8} & \theta^{2} & 1\end{array}$

Symmetry under $Z_{2}^{0}$ factor

\begin{tabular}{lllllllllllllllll}
1 & 1 & 1 & $\theta^{6}$ & $\theta^{6}$ & $\theta^{6}$ & $\theta^{6}$ & 1 & 1 & 1 & $\theta^{6}$ & $\theta^{6}$ & $\theta^{6}$ & 1 & 1 & 1 & 1 \\
\hline
\end{tabular} 
One can check that $\operatorname{det}\left(M_{D}\right)=0$, and that the resulting $M_{\nu}$ is singular and satisfies the texture $\mathbf{C}_{11}$.

As to $M_{\ell}$, we get a generic mass matrix:

$$
M_{\ell}=\left(\begin{array}{ccc}
Y_{\ell 11}^{1} v_{\Phi_{1}} & Y_{\ell 12}^{1} v_{\Phi_{1}} & Y_{\ell 13}^{1} v_{\Phi_{1}} \\
Y_{\ell 31}^{3} v_{\Phi_{2}}+Y_{\ell 21}^{3} v_{\Phi_{3}} & Y_{\ell 32}^{3} v_{\Phi_{2}}+Y_{\ell 22}^{3} v_{\Phi_{3}} & Y_{\ell 33}^{3} v_{\Phi_{2}}+Y_{\ell 23}^{3} v_{\Phi_{3}} \\
-Y_{\ell 21}^{3} v_{\Phi_{2}}+Y_{\ell 31}^{3} v_{\Phi_{3}} & -Y_{\ell 22}^{3} v_{\Phi_{2}}+Y_{\ell 32}^{3} v_{\Phi_{3}} & -Y_{\ell 23}^{3} v_{\Phi_{2}}+Y_{\ell 33}^{3} v_{\Phi_{3}}
\end{array}\right)
$$

Assuming the related VEVs are comparable $v_{\Phi_{1}} \approx v_{\Phi_{2}} \approx$ $v_{\Phi_{3}} \approx v$, then we get

$$
\begin{aligned}
M_{\ell} & \approx v\left(\begin{array}{ccc}
Y_{\ell 11}^{1} & Y_{\ell 12}^{1} & Y_{\ell 13}^{1} \\
Y_{\ell 31}^{3}+Y_{\ell 21}^{3} & Y_{\ell 32}^{3}+Y_{\ell 22}^{3} & Y_{\ell 33}^{3}+Y_{\ell 23}^{3} \\
-Y_{\ell 21}^{3}+Y_{\ell 31}^{3} & -Y_{\ell 22}^{3}+Y_{\ell 32}^{3} & -Y_{\ell 23}^{3}+Y_{\ell 33}^{3}
\end{array}\right) \\
& =v\left(\begin{array}{c}
\mathbf{a}^{T} \\
\mathbf{b}^{T} \\
\mathbf{c}^{T}
\end{array}\right),
\end{aligned}
$$

whereas if we assume $v \approx v_{\Phi_{1}} \approx v_{\Phi_{3}} \gg v_{\Phi_{2}}$, we get

$$
M_{\ell} \approx v\left(\begin{array}{lll}
Y_{\ell 11}^{1} & Y_{\ell 12}^{1} & Y_{\ell 13}^{1} \\
Y_{\ell 21}^{3} & Y_{\ell 22}^{3} & Y_{\ell 23}^{3} \\
Y_{\ell 31}^{3} & Y_{\ell 32}^{3} & Y_{\ell 33}^{3}
\end{array}\right)=v\left(\begin{array}{c}
\mathbf{a}^{T} \\
\mathbf{b}^{T} \\
\mathbf{c}^{T}
\end{array}\right)
$$

Again, in both cases, one can naturally diagonalize $M_{\ell}$ by an infinitesimal rotation, which means that we are to a good approximation in the flavor basis.

\section{Indirect realization of $\mathbf{C}_{22}$ (type I singular): Vanishing of $M_{\nu 11}+M_{\nu 33}$}

We move from zero texture at $(1,3)$ to the texture $\mathbf{C}_{22}$ by $S$ of Eq. (79). The symmetry transformations for the fields which impose a zero texture $M_{\nu 0}$ at entry $(1,3)$, with generic $M_{\ell 0}$ and singular $M_{D 0}$ are given in Table XIV.

Once more, we define the new transformations for the fields corresponding to the new symmetry imposing the vanishing subtrace by applying the rule in Eq. (66) with $S$ given by Eq. (79) or its extension $S_{e x}$ to the sevendimensional space of $\Phi$ 's. The rotated symmetry imposes some constraints on the Yukawa couplings and the VEVs, which when solved give the following results:

$$
\begin{aligned}
M_{R}= & \left(\begin{array}{ccc}
-Y_{\chi 13}^{3} v_{\chi_{1}}+Y_{\chi 11}^{3} v_{\chi_{3}} & 0 & Y_{\chi 11}^{3} v_{\chi_{1}}+Y_{\chi 13}^{3} v_{\chi_{3}} \\
0 & Y_{\chi 22}^{2} v_{\chi_{2}} & 0 \\
Y_{\chi 11}^{3} v_{\chi_{1}}+Y_{\chi 13}^{3} v_{\chi_{3}} & 0 & Y_{\chi 13}^{3} v_{\chi_{1}}-Y_{\chi 11}^{3} v_{\chi_{3}}
\end{array}\right), \\
M_{D}= & \left(\begin{array}{ccc}
Y_{D 33}^{7} v_{\Phi_{7}}+Y_{D 33}^{4} v_{\Phi_{4}} & 0 & -i Y_{D 33}^{7} v_{\Phi_{7}}+i Y_{D 33}^{4} v_{\Phi_{4}} \\
-i Y_{D 23}^{5} v_{\Phi_{5}}+i Y_{D 23}^{6} v_{\Phi_{6}} & 0 & Y_{D 23}^{6} v_{\Phi_{6}}+Y_{D 23}^{5} v_{\Phi_{5}} \\
i Y_{D 33}^{7} v_{\Phi_{7}}-i Y_{D 33}^{4} v_{\Phi_{4}} & 0 & Y_{D 33}^{7} v_{\Phi_{7}}+Y_{D 33}^{4} v_{\Phi_{4}}
\end{array}\right) .
\end{aligned}
$$

One can check that $\operatorname{det}\left(M_{D}\right)=0$, and that the resulting $M_{\nu}$ is singular and satisfies the texture $\mathbf{C}_{22}$.

As to $M_{\ell}$, we get a generic mass matrix:

$$
M_{\ell}=\left(\begin{array}{ccc}
Y_{\ell 31}^{3} v_{\Phi_{1}}+Y_{\ell 11}^{3} v_{\Phi_{3}} & Y_{\ell 32}^{3} v_{\Phi_{1}}+Y_{\ell 12}^{3} v_{\Phi_{3}} & Y_{\ell 33}^{3} v_{\Phi_{1}}+Y_{\ell 13}^{3} v_{\Phi_{3}} \\
Y_{\ell 21}^{2} v_{\Phi_{2}} & Y_{\ell 22}^{2} v_{\Phi_{2}} & Y_{\ell 23}^{2} v_{\Phi_{2}} \\
-Y_{\ell 11}^{3} v_{\Phi_{1}}+Y_{\ell 31}^{3} v_{\Phi_{3}} & -Y_{\ell 12}^{3} v_{\Phi_{1}}+Y_{\ell 32}^{3} v_{\Phi_{3}} & -Y_{\ell 13}^{3} v_{\Phi_{1}}+Y_{\ell 33}^{3} v_{\Phi_{3}}
\end{array}\right) .
$$

When $v_{\Phi_{1}} \approx v_{\Phi_{2}} \approx v_{\Phi_{3}} \approx v$, then we get

$$
M_{\ell} \approx v\left(\begin{array}{ccc}
Y_{\ell 31}^{3}+Y_{\ell 11}^{3} & Y_{\ell 32}^{3}+Y_{\ell 12}^{3} & Y_{\ell 33}^{3}+Y_{\ell 13}^{3} \\
Y_{\ell 21}^{2} & Y_{\ell 22}^{2} & Y_{\ell 23}^{2} \\
-Y_{\ell 11}^{3}+Y_{\ell 31}^{3} & -Y_{\ell 12}^{3}+Y_{\ell 32}^{3} & -Y_{\ell 13}^{3}+Y_{\ell 33}^{3}
\end{array}\right)=v\left(\begin{array}{c}
\mathbf{a}^{T} \\
\mathbf{b}^{T} \\
\mathbf{c}^{T}
\end{array}\right),
$$


TABLE XIV. The $Z_{12}^{0} \times Z_{2}^{0}$ symmetry realization of the one zero singular texture at the $(1,3)$ entry corresponding upon rotation to singular vanishing subtrace $\mathbf{C}_{22}$. The $D_{L 1}$ indicates the left-handed lepton doublet first family and so on. The $\chi_{k}$ denotes a scalar singlet which produces an entry in the righthanded Majorana mass matrix when acquiring a VEV at the seesaw scale. $\theta$ denotes $e^{i \pi / 6}$.

Symmetry under $Z_{12}^{0}$ factor

$\Phi_{1} \Phi_{2} \Phi_{3} \Phi_{4} \Phi_{5} \Phi_{6} \Phi_{7} D_{L 1} D_{L 2} D_{L 3} \nu_{R 1} \nu_{R 2} \nu_{R 3} \chi_{1} \chi_{2} \chi_{3} \ell_{R}$

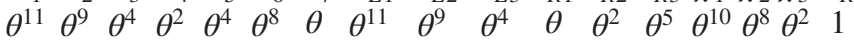
Symmetry under $Z_{2}^{0}$ factor

\begin{tabular}{lllllllllllllllll}
1 & 1 & 1 & $\theta^{6}$ & $\theta^{6}$ & $\theta^{6}$ & $\theta^{6}$ & 1 & 1 & 1 & $\theta^{6}$ & $\theta^{6}$ & $\theta^{6}$ & 1 & 1 & 1 & 1 \\
\hline \hline
\end{tabular}

whereas when $v \approx v_{\Phi_{3}} \approx v_{\Phi_{2}} \gg v_{\Phi_{1}}$, we get

$$
M_{\ell} \approx v\left(\begin{array}{lll}
Y_{\ell 11}^{3} & Y_{\ell 12}^{3} & Y_{\ell 13}^{3} \\
Y_{\ell 21}^{2} & Y_{\ell 22}^{2} & Y_{\ell 23}^{2} \\
Y_{\ell 31}^{3} & Y_{\ell 32}^{3} & Y_{\ell 33}^{3}
\end{array}\right)=v\left(\begin{array}{c}
\mathbf{a}^{T} \\
\mathbf{b}^{T} \\
\mathbf{c}^{T}
\end{array}\right)
$$

In both cases, one can naturally diagonalize $M_{\ell}$ by an infinitesimal rotation, which means that we are approximately in the flavor basis.
TABLE XV. The $Z_{12}^{0} \times Z_{2}^{0}$ symmetry realization of the one zero singular texture at the $(2,3)$ entry corresponding upon rotation to singular vanishing subtrace $\mathbf{C}_{31}$. The $D_{L 1}$ indicates the lefthanded lepton doublet first family and so on. The $\chi_{k}$ denotes a scalar singlet which produces an entry in the right-handed Majorana mass matrix when acquiring a VEV at the seesaw scale. $\theta$ denotes $e^{i \pi / 6}$.

Symmetry under $Z_{12}^{0}$ factor

$\Phi_{1} \Phi_{2} \Phi_{3} \Phi_{4} \Phi_{5} \Phi_{6} \Phi_{7} D_{L 1} D_{L 2} D_{L 3} \nu_{R 1} \nu_{R 2} \nu_{R 3} \chi_{1} \chi_{2} \chi_{3} \ell_{R}$

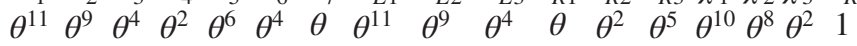
Symmetry under $Z_{2}^{0}$ factor

\begin{tabular}{lllllllllllllllll}
1 & 1 & 1 & $\theta^{6}$ & $\theta^{6}$ & $\theta^{6}$ & $\theta^{6}$ & 1 & 1 & 1 & $\theta^{6}$ & $\theta^{6}$ & $\theta^{6}$ & 1 & 1 & 1 & 1 \\
\hline
\end{tabular}

\section{Indirect realization of $\mathbf{C}_{31}$ (type $\mathbf{I}$ singular): Vanishing of $M_{\nu 12}+M_{\nu 23}$}

We move from zero texture at $(2,3)$ to the texture $\mathbf{C}_{31}$ by $S$ of Eq. (84). The symmetry transformations for the fields which imposes a zero texture at entry $(2,3)$ of $M_{\nu 0}$ with generic $M_{\ell}$ and singular $M_{D 0}$ are given in Table XV.

In order to define the new transformations for the fields corresponding to the new symmetry imposing the vanishing subtrace, we apply the rule of Eq. (66) with $S$ given by Eq. (84) or its extension $S_{e x}$ to the seven-dimensional space of $\Phi$ 's. Solving the constraints on the Yukawa couplings and the VEVs resulting from the rotated symmetry, we get

$$
\begin{aligned}
M_{R}= & \left(\begin{array}{cccc}
Y_{\chi 11}^{1} v_{\chi_{1}}+Y_{\chi 13}^{1} v_{\chi_{3}} & 0 & Y_{\chi 13}^{1} v_{\chi_{1}}+Y_{\chi 11}^{1} v_{\chi_{3}} \\
0 & Y_{\chi 22}^{2} v_{\chi_{2}} & 0 \\
Y_{\chi 13}^{1} v_{\chi_{1}}+Y_{\chi 11}^{1} v_{\chi_{3}} & 0 & Y_{\chi 11}^{1} v_{\chi_{1}}+Y_{\chi 13}^{1} v_{\chi_{3}}
\end{array}\right), \\
M_{D}= & \left(\begin{array}{cccc}
Y_{D 33}^{4} v_{\Phi_{4}}-Y_{D 31}^{5} v_{\Phi_{5}}+Y_{D 33}^{7} v_{\Phi_{7}} & 0 & -Y_{D 33}^{4} v_{\Phi_{4}}-Y_{D 31}^{5} v_{\Phi_{5}}+Y_{D 33}^{7} v_{\Phi_{7}} \\
-Y_{D 23}^{6} v_{\Phi_{6}} & 0 & Y_{D 23}^{6} v_{\Phi_{6}} \\
-Y_{D 33}^{4} v_{\Phi_{4}}+Y_{D 31}^{5} v_{\Phi_{5}}+Y_{D 33}^{7} v_{\Phi_{7}} & 0 & Y_{D 33}^{4} v_{\Phi_{4}}+Y_{D 31}^{5} v_{\Phi_{5}}+Y_{D 33}^{7} v_{\Phi_{7}}
\end{array}\right) .
\end{aligned}
$$

One can check that $\operatorname{det}\left(M_{D}\right)=0$, and that the resulting $M_{\nu}$ is singular and satisfies the texture $\mathbf{C}_{31}$.

As to $M_{\ell}$, we get a generic mass matrix:

$$
M_{\ell}=\left(\begin{array}{ccc}
Y_{\ell 31}^{3} v_{\Phi_{1}}+Y_{\ell 11}^{3} v_{\Phi_{3}} & Y_{\ell 32}^{3} v_{\Phi_{1}}+Y_{\ell 12}^{3} v_{\Phi_{3}} & Y_{\ell 33}^{3} v_{\Phi_{1}}+Y_{\ell 13}^{3} v_{\Phi_{3}} \\
Y_{\ell 21}^{2} v_{\Phi_{2}} & Y_{\ell 22}^{2} v_{\Phi_{2}} & Y_{\ell 23}^{2} v_{\Phi_{2}} \\
Y_{\ell 11}^{3} v_{\Phi_{1}}+Y_{\ell 31}^{3} v_{\Phi_{3}} & Y_{\ell 12}^{3} v_{\Phi_{1}}+Y_{\ell 32}^{3} v_{\Phi_{3}} & Y_{\ell 13}^{3} v_{\Phi_{1}}+Y_{\ell 33}^{3} v_{\Phi_{3}}
\end{array}\right) .
$$

When $v \approx v_{\Phi_{1}} \approx v_{\Phi_{2}} \gg v_{\Phi_{3}}$, then we get

whereas if we assume $v \approx v_{\Phi_{3}} \approx v_{\Phi_{2}} \gg v_{\Phi_{1}}$, we get

$$
M_{\ell} \approx v\left(\begin{array}{lll}
Y_{\ell 31}^{3} & Y_{\ell 32}^{3} & Y_{\ell 33}^{3} \\
Y_{\ell 21}^{2} & Y_{\ell 22}^{2} & Y_{\ell 23}^{2} \\
Y_{\ell 11}^{3} & Y_{\ell 12}^{3} & Y_{\ell 13}^{3}
\end{array}\right)=v\left(\begin{array}{c}
\mathbf{a}^{T} \\
\mathbf{b}^{T} \\
\mathbf{c}^{T}
\end{array}\right),
$$

$$
M_{\ell} \approx v\left(\begin{array}{lll}
Y_{\ell 11}^{3} & Y_{\ell 12}^{3} & Y_{\ell 13}^{3} \\
Y_{\ell 21}^{2} & Y_{\ell 22}^{2} & Y_{\ell 23}^{2} \\
Y_{\ell 31}^{3} & Y_{\ell 32}^{3} & Y_{\ell 33}^{3}
\end{array}\right)=v\left(\begin{array}{c}
\mathbf{a}^{T} \\
\mathbf{b}^{T} \\
\mathbf{c}^{T}
\end{array}\right)
$$


In both cases, one can naturally diagonalize $M_{\ell}$ by an infinitesimal rotation, which means that we are in the flavor basis approximately.

\section{INDIRECT REALIZATION OF TYPE II SEESAW WITH $Z_{5}$ SYMMETRY}

To fix the ideas, we treat here in some details the case of the $\mathbf{C}_{33}$ vanishing subtrace which can be related to zero texture, noting that the procedure can be generalized to all other textures $\left(\mathbf{C}_{11}, \mathbf{C}_{22}\right.$, and $\left.\mathbf{C}_{31}\right)$ that also can be related to zero textures. We follow the same rotating strategy outlined in Sec. VII.

As we saw in Eq. (57), the matrix $S$ allows one to move from one zero texture at the $(1,2)$ th entry to the vanishing subtrace texture $\mathbf{C}_{33}$. Again, we use a subscript (or superscript) 0 to denote the gauge basis satisfying the unrotated' symmetry $Z_{5}^{0}$, whereas we drop this subscript (superscript) for the rotated $Z_{5}$.

\section{A. Matter content}

Following the conventions of [5], we extend the SM extended by introducing several $S U(2)_{L}$ scalar triplets $H_{a}$, $(a=1,2, \ldots, N)$,

$$
H_{a} \equiv\left[H_{a}^{++}, H_{a}^{+}, H_{a}^{0}\right] .
$$

The gauge invariant Yukawa interaction relevant for neutrino mass takes the form

$$
\begin{aligned}
\mathcal{L}_{H, L}= & \sum_{i, j=1}^{3} \sum_{a=1}^{N} Y_{i j}^{\nu a}\left[H_{a}^{0} \nu_{L i}^{T} \mathcal{C}^{-1} \nu_{L j}\right. \\
& +H_{a}^{+}\left(\nu_{L i}^{T} \mathcal{C}^{-1} \ell_{L j}+\ell_{L j}^{T} \mathcal{C}^{-1} \nu_{L i}\right) \\
& \left.+H_{a}^{++} \ell_{L i}^{T} \mathcal{C}^{-1} \ell_{L j}\right],
\end{aligned}
$$

where $Y_{i j}^{a}$ are the corresponding Yukawa coupling constants, the indices $i, j$ are flavor ones, and $\mathcal{C}$ is the charge conjugation matrix.

The field $H_{a}^{0}$ could acquire a small VEV, $\left\langle H_{a}^{0}\right\rangle=v_{a}^{H}$, that gives rise to a Majorana neutrino mass matrix of the following form:

$$
M_{\nu i j}=\sum_{a=1}^{N} Y_{i j}^{\nu a} v_{a}^{H} .
$$

The smallness of the VEV $v_{a}^{H}$ is attributed to the largeness of the triplet scalar mass scale [20].

As to the charged lepton mass, we introduce, in contrast to [5], various Higgs doublets $\Phi_{a}, a=1, \ldots, K$,

$$
\mathcal{L}_{\ell}=\sum_{i, j=1}^{3} \sum_{a=1}^{K} Y_{i j}^{\ell a} \bar{D}_{L i} \Phi_{a} \ell_{R j} .
$$

Note that we did not consider only one SM Higgs; otherwise we would have got, as in [5], a diagonal charged lepton mass matrix $M_{\ell 0}$ when the neutrino mass matrix $M_{\nu 0}$ had a zero texture. We would like to get a generic $M_{\ell 0}$ corresponding to zero texture $M_{\nu 0}$, so that when we rotate and get a vanishing subtrace texture for the neutrino mass matrix $M_{\nu}$ we also get another generic charged lepton mass matrix $M_{\ell}$. This latter can under suitable assumptions be diagonalized by infinitesimal rotations. Had we restricted our SM Higgs to only one Higgs doublet, then the diagonal $M_{\ell 0}$ corresponding to zero texture $M_{\nu 0}$ will give, upon rotation by $S$, a nondiagonal charged mass matrix $M_{\ell}$ that is diagonalizable by a finite rotation $S$, which means that the vanishing subtrace texture does not correspond to the flavor basis.

\section{B. $Z_{5}^{0}$ symmetry for zero texture $M_{\nu 0}$ characterized by $M_{\nu 012}=0$}

In order to impose a zero texture $M_{\nu 0}$ by $Z_{5}^{0}$ symmetry with a generic $M_{\ell 0}$, we introduce four scalar triplets $H_{a}$ and three Higgs doublets $\Phi_{b}$, with the following assignments under $Z_{5}^{0}$ defined in Table XVI.

By forming bilinear terms of $H_{a} \nu_{L}^{T} \nu_{L}$ we can find out the invariant Lagrangian terms under $Z_{5}^{0}$, which gives

$$
M_{\nu 0}=\left(\begin{array}{ccc}
\times & 0 & \times \\
0 & \times & \times \\
\times & \times & \times
\end{array}\right) .
$$

\section{C. $Z_{5}$ symmetry for $\mathrm{C}_{33}$ texture $\left(M_{\nu 11}+M_{\nu 22}=0\right)$ and Yukawa couplings constraints}

In order to find the new rotated symmetry $Z_{5}$, we need first to find how all the fields would transform. Here, we carefully use the rule of [Eq. (46) or (52)] for all the fields $f$, in that if $f$ transforms under $Z_{5}^{0}$ according to the diagonal, by construction, matrix $T_{f}^{0 Z}$, then it transforms under $Z_{5}$ according to $T_{f}^{Z}=S_{e x}^{f \dagger} T_{f}^{0 Z} S_{e x}^{f}$ [cf. Eq. (66)] with $S_{e x}^{f}=\operatorname{diag}\left(S, 1_{r \times r}\right)$ possibly an extension of $S$ to match the finite-dimensional space of the field $f$ of dimensions $(3+r)$. The invariance of the Lagrangian terms under

TABLE XVI. The $Z_{5}^{0}$ symmetry seesaw type II realization of the one zero texture at the $(1,2)$ entry corresponding upon rotation to vanishing subtrace $\mathbf{C}_{33} . H_{a}$ are triplet scalars, whereas $1_{F}$ refers to the fermions, apart from the right-handed charged leptons $\ell_{R}$, in the first generation and so on. The $\Phi_{b}$ denote SM Higgs doublets. $\Omega$ denotes $e^{i 2 \pi / 5}$.

Symmetry under $Z_{5}^{0}$

$\begin{array}{lllllllllll}H_{1} & H_{2} & H_{3} & H_{4} & 1_{F} & 2_{F} & 3_{F} & \ell_{R} & \Phi_{1} & \Phi_{2} & \Phi_{3}\end{array}$

\begin{tabular}{lllllllllll}
1 & $\Omega^{3}$ & $\Omega^{2}$ & $\Omega$ & 1 & $\Omega$ & $\Omega^{2}$ & 1 & 1 & $\Omega$ & $\Omega^{2}$ \\
\hline \hline
\end{tabular}


the symmetry will impose constraints on the Yukawa couplings that one can in principle solve to give the form of the mass matrices when the Higgs/scalar fields get a VEV.

Actually, one can check that under both $Z_{5}^{0}$ and $Z_{5}$, defined by the transformations $T_{f}^{0 Z}$ and $T_{f}^{Z}$, respectively, we have the following constraints:

$$
\left(Y_{0}^{\nu b}\right)=T_{H a b}^{0 Z}\left(T_{\nu_{L}}^{0 Z}\right)^{T}\left(Y_{0}^{\nu a}\right)\left(T_{\nu_{L}}^{0 Z}\right)
$$

and

$$
\left(Y^{\nu b}\right)=T_{H a b}^{Z}\left(T_{\nu_{L}}^{Z}\right)^{T}\left(Y^{\nu a}\right)\left(T_{\nu_{L}}^{Z}\right),
$$

where $(a, b=1, \ldots, 4),\left(Y_{0}^{\nu b}\right)$ is a matrix in flavor space with element $Y_{0 i j}^{\nu b}$ at its $(i, j)$ th entry. The two constraints of
Eqs. (111) and (112) are related in that if we know the solution to one constraint we know it for the other. More specifically, one can check that if $\left(Y_{0}^{\nu b}\right)$ was a solution of Eq. (111), then

$$
\left(Y^{\nu e}\right)=\left(S^{T}\right)\left(Y_{0}^{\nu b}\right)(S)\left(S_{e x}^{H}\right)_{b e}
$$

is a solution of Eq. (112).

\section{D. $M_{\nu 0}$ and $M_{\nu}$ resulting, respectively, from $Z_{5}^{0}$ and $Z_{5}$ invariance}

By solving Eqs. (111) and (112) we get when $H_{a}^{0}$ 's get the VEVs $v_{0 a}^{H}$ under $Z_{5}^{0}$ :

$$
\begin{aligned}
M_{\nu 0} & =\left(\begin{array}{ccc}
Y_{011}^{\nu 1} v_{01}^{H} & 0 & Y_{013}^{\nu 2} v_{02}^{H} \\
- & Y_{022}^{\nu 2} v_{02}^{H} & Y_{023}^{\nu 3} v_{03}^{H} \\
- & - & Y_{033}^{\nu 4} v_{04}^{H}
\end{array}\right), \\
M_{\nu} & =\left(\begin{array}{ccc}
-Y_{23}^{\nu 2} v_{1}^{H}-Y_{22}^{\nu 2} v_{2}^{H} & -Y_{22}^{\nu 2} v_{1}^{H}+Y_{12}^{\nu 2} v_{2}^{H} & Y_{23}^{\nu 2} v_{1}^{H}-i Y_{23}^{\nu 2} v_{2}^{H}+i Y_{23}^{\nu 3} v_{3}^{H} \\
- & Y_{12}^{\nu 2} v_{1}^{H}+Y_{22}^{\nu 2} v_{2}^{H} & i Y_{23}^{\nu 2} v_{1}^{H}+Y_{23}^{\nu 2} v_{2}^{H}+Y_{23}^{\nu 3} v_{3}^{H} \\
- & - & Y_{33}^{\nu 4} v_{4}^{H}
\end{array}\right) .
\end{aligned}
$$

We see that the texture $\mathbf{C}_{33}$ is met in $M_{\nu}$ while $\left(M_{\nu 012}=0\right)$ for $M_{\nu 0}$. One can deduce the relations between the Higgs VEVs in the unrotated system $\left(v_{0 a}^{H}\right)$ and the Higgs VEVs in the rotated system $\left(v_{a}^{H}\right)$ by writing Eq. (69) and considering Eq. (113).

\section{E. $M_{\ell 0}$ and $M_{\ell}$ resulting, respectively, from $Z_{5}^{0}$ and $Z_{5}$ invariance}

The introduction of three SM Higgs $\Phi$ 's was needed essentially to produce a generic charged lepton matrix. Actually the bilinear of the relevant term $\bar{D}_{L i} \ell_{R j}$ transforms under $Z_{5}^{0}$ as

$$
\bar{D}_{L i} \ell_{R j} \cong\left(\begin{array}{ccc}
1 & 1 & 1 \\
\Omega^{4} & \Omega^{4} & \Omega^{4} \\
\Omega^{3} & \Omega^{3} & \Omega^{3}
\end{array}\right) .
$$

We see now that the transformations of $\Phi$ 's in Table XVI were chosen exactly to make all the entries in $M_{\ell 0}$ eligible. Again expressing the invariance under $Z_{5}^{0}$ gives constraints on the Yukawa couplings:

$$
\left(Y_{0}^{\ell b}\right)=T_{\Phi a b}^{0 Z}\left(T_{D_{L}}^{0 Z}\right)^{\dagger}\left(Y_{0}^{\ell a}\right)\left(T_{\ell_{R}}^{0 Z}\right)
$$

and

$$
\left(Y^{\ell b}\right)=T_{\Phi a b}^{Z}\left(T_{D_{L}}^{Z}\right)^{\dagger}\left(Y^{\ell a}\right)\left(T_{\ell_{R}}^{Z}\right)
$$

where $(a, b=1, \ldots, 3)$ and $\left(Y_{0}^{\ell b}\right)$ is a matrix in flavor space with element $Y_{0 i j}^{\ell b}$ at its $(i, j)$ th entry. The two constraints of Eqs. (116) and (117) are related in that if $\left(Y_{0}^{\ell b}\right)$ was a solution of Eq. (116), then

$$
\left(Y^{\ell g}\right)=\left(S^{\dagger}\right)\left(Y_{0}^{\ell b}\right)(S)\left(S_{e x}^{\Phi}\right)_{b g}
$$

is a solution of Eq. (117) where $S_{e x}^{\Phi}=S$ since we have three $\Phi$ 's.

Solving the Yukawa constraints in Eqs. (116) and (117) we see that when the $\Phi_{a}$ 's get VEVs $v_{0 a}^{\Phi}$, we get the following $M_{\ell 0}$ and $M_{\ell}$ :

$$
M_{\ell 0}=\left(\begin{array}{ccc}
Y_{011}^{\ell 1} v_{01}^{\Phi} & Y_{012}^{\ell 1} v_{01}^{\Phi} & Y_{013}^{\ell 1} v_{01}^{\Phi} \\
Y_{021}^{\ell 2} v_{02}^{\Phi} & Y_{022}^{\ell 2} v_{02}^{\Phi} & Y_{023}^{\ell 2} v_{02}^{\Phi} \\
Y_{031}^{\ell 3} v_{03}^{\Phi} & Y_{032}^{\ell 3} v_{03}^{\Phi} & Y_{033}^{\ell 3} v_{03}^{\Phi}
\end{array}\right)
$$

and 


$$
M_{\ell}=\left(\begin{array}{ccc}
Y_{21}^{\ell 2} v_{1}^{\Phi}+Y_{11}^{\ell 2} v_{2}^{\Phi} & Y_{22}^{\ell 2} v_{1}^{\Phi}+Y_{12}^{\ell 2} v_{2}^{\Phi} & Y_{23}^{\ell 2} v_{1}^{\Phi}+Y_{13}^{\ell 2} v_{2}^{\Phi} \\
-Y_{11}^{\ell 2} v_{1}^{\Phi}+Y_{21}^{\ell 2} v_{2}^{\Phi} & -Y_{12}^{\ell 2} v_{1}^{\Phi}+Y_{22}^{\ell 2} v_{2}^{\Phi} & -Y_{13}^{\ell 2} v_{1}^{\Phi}+Y_{23}^{\ell 2} v_{2}^{\Phi} \\
Y_{31}^{\ell 3} v_{3}^{\Phi} & Y_{31}^{\ell 3} v_{3}^{\Phi} & Y_{33}^{\ell 3} v_{3}^{\Phi}
\end{array}\right)
$$

Again, one can deduce the relations between the unrotated VEVs $\left(v_{o a}^{\Phi}\right)$ and the rotated VEVs $\left(v_{a}^{\Phi}\right)$ by writing [cf. Eq. (69)] $M_{\ell}=S^{\dagger} \cdot M_{\ell o} \cdot S$ and considering Eq. (118).

Thus if we assume the related VEVs are comparable $v_{\Phi_{1}} \approx v_{\Phi_{2}} \approx v_{\Phi_{3}} \approx v$, then we get

$$
\begin{aligned}
M_{\ell} & \approx v\left(\begin{array}{ccc}
Y_{21}^{\ell 2}+Y_{11}^{\ell 2} & Y_{22}^{\ell 2}+Y_{12}^{\ell 2} & Y_{23}^{\ell 2}+Y_{13}^{\ell 2} \\
-Y_{11}^{\ell 2}+Y_{21}^{\ell 2} & -Y_{12}^{\ell 2}+Y_{22}^{\ell 2} & -Y_{13}^{\ell 2}+Y_{23}^{\ell 2} \\
Y_{31}^{\ell 3} & Y_{31}^{\ell 3} & Y_{33}^{\ell 3}
\end{array}\right) \\
& =v\left(\begin{array}{l}
\mathbf{a}^{T} \\
\mathbf{b}^{T} \\
\mathbf{c}^{T}
\end{array}\right) .
\end{aligned}
$$

Consequently,

$$
M_{\ell} M_{\ell}^{\dagger} \approx v^{2}\left(\begin{array}{lll}
\mathbf{a} \cdot \mathbf{a} & \mathbf{a} \cdot \mathbf{b} & \mathbf{a} \cdot \mathbf{c} \\
\mathbf{b} \cdot \mathbf{a} & \mathbf{b} \cdot \mathbf{b} & \mathbf{b} \cdot \mathbf{c} \\
\mathbf{c} \cdot \mathbf{a} & \mathbf{c} \cdot \mathbf{b} & \mathbf{c} \cdot \mathbf{c}
\end{array}\right)
$$

so taking only the following natural assumption on the norms of the vectors

$$
\begin{aligned}
& \|\mathbf{a}\| /\|\mathbf{c}\|=m_{e} / m_{\tau} \sim 3 \times 10^{-4}, \\
& \|\mathbf{b}\| /\|\mathbf{c}\|=m_{\mu} / m_{\tau} \sim 6 \times 10^{-2},
\end{aligned}
$$

one can diagonalize $M_{\ell} M_{\ell}^{\dagger}$ by an infinitesimal rotation as was done in [5], which proves that we are to a good approximation in the flavor basis.

\section{DIRECT REALIZATION OF TYPE I SEESAW WITH $Z_{6} \times Z_{2}$ SYMMETRY}

We present now another method that leads directly to the vanishing subtrace texture without relating it to zero textures by rotation. It is applicable again only for the four textures $\left(\mathbf{C}_{33}, \mathbf{C}_{11}, \mathbf{C}_{22}\right.$, and $\left.\mathbf{C}_{31}\right)$.

\section{A. Type I seesaw direct realization of $\mathrm{C}_{33}$ : Vanishing of $M_{\nu 11}+M_{\nu 22}$}

Within the type I seesaw scenario, the Lagrangian responsible for mass is similar to the one given in Eq. (58) which, after conveniently simplifying the notations by dropping the Yukawa 0 -subscript and the summation signs, is rewritten here as

$$
\begin{aligned}
\mathcal{L}_{M} \supset & Y_{\chi i j}^{b} \chi_{b} \nu_{R i}^{T} \mathcal{C}^{-1} \nu_{R j}+Y_{D i j}^{a} \bar{D}_{L i} \tilde{\Phi}_{a} \nu_{R j} \\
& +Y_{\ell i j}^{a} \bar{D}_{L i} \Phi_{a} \ell_{R j} .
\end{aligned}
$$

We have for the pattern $\mathbf{C}_{33}$ the relation $M_{\nu 11}+M_{\nu 22}=0$, which can give a hint motivating the search for solutions involving a permutation symmetry $(1 \leftrightarrow 2)$. Actually, we can think of the vanishing subtrace constraint as arising from symmetry considerations leading to textures implementing these "permutation"restrictions at the level of $M_{R}$ and $M_{D}$, which by the seesaw scenario resurface at the level of $M_{\nu}$ which inherits the permutation structure. One can try simple forms for both $M_{R}$ and $M_{D}$ with enough parameters in order to produce generic $M_{\nu}$ having the sole constraint $M_{\nu 11}+M_{\nu 22}=0$. To be concrete, one can assume the following forms for $M_{R}$ and $M_{D}$ as shown below together with the derived $M_{\nu}$ (through the seesaw mechanism),

$M_{R}=\left(\begin{array}{ccc}x & y & 0 \\ y & -x & 0 \\ 0 & 0 & z\end{array}\right), \quad M_{D}=\left(\begin{array}{ccc}A & -B & i C \\ B & A & -C \\ -i D & D & E\end{array}\right)$,

$M_{\nu}=M_{D} M_{R}^{-1} M_{D}^{T}=\left(\begin{array}{ccc}\Delta & \times & \times \\ - & -\Delta & \times \\ - & - & \times\end{array}\right)$,

where $A, B, C, D, E, x, y$, and $z$ are generic independent parameters and the $\times$ and $\Delta$ signs denote generic independent nonvanishing entries. We stress here that these forms proposed for $M_{R}$ and $M_{D}$ are not necessarily the simplest choices, but they are just mere possibilities that can be derived from symmetry considerations.

The fields and their assigned symmetry transformations under $Z_{6} \times Z_{2}$ are presented in Table XVII.

Forming the required bilinears dictated by $Z_{6}$ symmetry, we obtain

$$
\begin{aligned}
\nu_{R i}^{T} \nu_{R j} \stackrel{Z_{6}}{\cong}\left(\begin{array}{ccc}
1 & 1 & \omega \\
1 & 1 & \omega \\
\omega & \omega & \omega^{2}
\end{array}\right), \quad \bar{D}_{L i} \nu_{R j} \stackrel{Z_{6}}{\cong}\left(\begin{array}{ccc}
1 & 1 & \omega \\
1 & 1 & \omega \\
\omega^{5} & \omega^{5} & 1
\end{array}\right), \\
\bar{D}_{L i} \ell_{R j} \stackrel{Z_{6}}{\cong}\left(\begin{array}{ccc}
-1 & -1 & \omega^{4} \\
-1 & -1 & \omega^{4} \\
\omega^{2} & \omega^{2} & -1
\end{array}\right) .
\end{aligned}
$$

When the resulting bilinears combine with the appropriate scalar fields, we get under $Z_{6}$, keeping only the combinations that produce singlets, the following: 
TABLE XVII. The $Z_{6} \times Z_{2}$ symmetry seesaw type I realization of the vanishing subtrace $\mathbf{C}_{33} . \Phi$ are five SM Higgs doublets, $D_{L}$ refers to the flavor three left-handed lepton doublets, while the three right-handed charged lepton singlets are denoted by $\ell_{R} . \omega$ denotes $e^{i \pi / 3}$.

Matter content and symmetry transformation (pattern $\mathbf{C}_{33}$ )

Symmetry under $Z_{6}$

\begin{tabular}{lccccc}
\hline$\nu_{R 1} \rightarrow \nu_{R 1}$ & $\nu_{R 2} \rightarrow \nu_{R 2}$ & $\nu_{R 3} \rightarrow \omega \nu_{R 3}$ & $\chi_{1} \rightarrow \omega^{4} \chi_{1}$ & $\chi_{2} \rightarrow \chi_{2}$ & $\chi_{3} \rightarrow \chi_{3}$ \\
$D_{L 1} \rightarrow D_{L 1}$ & $D_{L 2} \rightarrow D_{L 2}$ & $D_{L 3} \rightarrow \omega D_{L 3}$ & $\Phi_{1} \rightarrow \Phi_{1}$ & $\Phi_{2} \rightarrow \omega \Phi_{2}$ & $\Phi_{3} \rightarrow \omega^{5} \Phi_{3}$ \\
$\Phi_{4} \rightarrow \omega^{3} \Phi_{4}$ & $\Phi_{5} \rightarrow \omega^{3} \Phi_{5}$ & $\ell_{R 1} \rightarrow \omega^{3} \ell_{R 1}$ & $\ell_{R 2} \rightarrow \omega^{3} \ell_{R 2}$ & $\ell_{R 3} \rightarrow \omega^{4} \ell_{R 3}$ &
\end{tabular}

Symmetry under $Z_{2}$

\begin{tabular}{lccccc}
$\nu_{R 1} \rightarrow i \nu_{R 2}$ & $\nu_{R 2} \rightarrow-i \nu_{R 1}$ & $\nu_{R 3} \rightarrow \nu_{R 3}$ & $\chi_{1} \rightarrow \chi_{1}$ & $\chi_{2} \rightarrow \chi_{2}$ & $\chi_{3} \rightarrow \chi_{3}$ \\
$D_{L 1} \rightarrow i D_{L 2}$ & $D_{L 2} \rightarrow-i D_{L 1}$ & $D_{L 3} \rightarrow D_{L 3}$ & $\Phi_{1} \rightarrow \Phi_{1}$ & $\Phi_{2} \rightarrow-\Phi_{2}$ & $\Phi_{3} \rightarrow \Phi_{3}$ \\
$\Phi_{4} \rightarrow i \Phi_{5}$ & $\Phi_{5} \rightarrow-i \Phi_{4}$ & $\ell_{R 1} \rightarrow \ell_{R 1}$ & $\ell_{R 2} \rightarrow \ell_{R 2}$ & $\ell_{R 3} \rightarrow \ell_{R 3}$ & \\
\hline \hline
\end{tabular}

$$
\begin{aligned}
& \chi_{1} \nu_{R i}^{T} \nu_{R j} \stackrel{Z_{6}}{\cong}\left(\begin{array}{ccc}
\omega^{4} & \omega^{4} & \omega^{5} \\
\omega^{4} & \omega^{4} & \omega^{5} \\
\omega^{5} & \omega^{5} & 1
\end{array}\right), \quad \chi_{2} \nu_{R i}^{T} \nu_{R j} \cong\left(\begin{array}{ccc}
1 & 1 & \omega \\
1 & 1 & \omega \\
\omega & \omega & \omega^{2}
\end{array}\right), \quad \chi_{3} \nu_{R i}^{T} \nu_{R j} \cong\left(\begin{array}{ccc}
1 & 1 & \omega \\
1 & 1 & \omega \\
\omega & \omega & \omega^{2}
\end{array}\right), \\
& \tilde{\Phi}_{1} \bar{D}_{L i} \nu_{R j} \stackrel{Z_{6}}{\cong}\left(\begin{array}{ccc}
1 & 1 & \omega \\
1 & 1 & \omega \\
\omega^{5} & \omega^{5} & 1
\end{array}\right), \quad \tilde{\Phi}_{2} \bar{D}_{L i} \nu_{R j} \stackrel{Z_{6}}{\cong}\left(\begin{array}{ccc}
\omega^{5} & \omega^{5} & 1 \\
\omega^{5} & \omega^{5} & 1 \\
\omega^{4} & \omega^{4} & \omega^{5}
\end{array}\right), \quad \tilde{\Phi}_{3} \bar{D}_{L i} \nu_{R j} \cong\left(\begin{array}{ccc}
Z_{6} & \omega & \omega^{2} \\
\omega & \omega & \omega^{2} \\
1 & 1 & \omega^{5}
\end{array}\right), \\
& \Phi_{4} \bar{D}_{L i} \ell_{R j} \stackrel{Z_{6}}{\cong}\left(\begin{array}{ccc}
1 & 1 & \omega \\
1 & 1 & \omega \\
\omega^{5} & \omega^{5} & 1
\end{array}\right), \quad \Phi_{5} \bar{D}_{L i} \ell_{R j} \stackrel{Z_{6}}{\cong}\left(\begin{array}{ccc}
1 & 1 & \omega \\
1 & 1 & \omega \\
\omega^{5} & \omega^{5} & 1
\end{array}\right) .
\end{aligned}
$$

Thus the resulting Lagrangian dictated by $Z_{6}$ symmetry takes the form

$$
\begin{aligned}
\mathcal{L}_{M}^{Z_{6}} \propto & Y_{\chi 33}^{1} \chi_{1} \nu_{R 3}^{T} \mathcal{C}^{-1} \nu_{R 3} \\
& +Y_{\chi 11}^{2} \chi_{2} \nu_{R 1}^{T} \mathcal{C}^{-1} \nu_{R 1}+Y_{\chi 12}^{2} \chi_{2} \nu_{R 1}^{T} \mathcal{C}^{-1} \nu_{R 2}+Y_{\chi 12}^{2} \chi_{2} \nu_{R 2}^{T} \mathcal{C}^{-1} \nu_{R 1}+Y_{\chi 22}^{2} \chi_{2} \nu_{R 2}^{T} \mathcal{C}^{-1} \nu_{R 2} \\
& +Y_{\chi 11}^{3} \chi_{3} \nu_{R 1}^{T} \mathcal{C}^{-1} \nu_{R 1}+Y_{\chi 12}^{3} \chi_{3} \nu_{R 1}^{T} \mathcal{C}^{-1} \nu_{R 2}+Y_{\chi 12}^{3} \chi_{3} \nu_{R 2}^{T} \mathcal{C}^{-1} \nu_{R 1}+Y_{\chi 22}^{3} \chi_{3} \nu_{R 2}^{T} \mathcal{C}^{-1} \nu_{R 2} \\
& +Y_{D 11}^{1} \bar{D}_{L 1} \tilde{\Phi}_{1} \nu_{R 1}+Y_{D 12}^{1} \bar{D}_{L 1} \tilde{\Phi}_{1} \nu_{R 2}+Y_{D 21}^{1} \bar{D}_{L 2} \tilde{\Phi}_{1} \nu_{R 1}+Y_{D 22}^{1} \bar{D}_{L 2} \tilde{\Phi}_{1} \nu_{R 2}+Y_{D 33}^{1} \bar{D}_{L 3} \tilde{\Phi}_{1} \nu_{R 3} \\
& +Y_{D 13}^{2} \bar{D}_{L 1} \tilde{\Phi}_{2} \nu_{R 3}+Y_{D 23}^{2} \bar{D}_{L 2} \tilde{\Phi}_{2} \nu_{R 3}+Y_{D 31}^{3} \bar{D}_{L 3} \tilde{\Phi}_{3} \nu_{R 1}+Y_{D 32}^{3} \bar{D}_{L 3} \tilde{\Phi}_{3} \nu_{R 2} \\
& +Y_{\ell 11}^{4} \bar{D}_{L 1} \Phi_{4} \ell_{R 1}+Y_{\ell 12}^{4} \bar{D}_{L 1} \Phi_{4} \ell_{R 2}+Y_{\ell 21}^{4} \bar{D}_{L 2} \Phi_{4} \ell_{R 1}+Y_{\ell 22}^{4} \bar{D}_{L 2} \Phi_{4} \ell_{R 2}+Y_{\ell 33}^{4} \bar{D}_{L 3} \Phi_{4} \ell_{R 3} \\
& +Y_{\ell 11}^{5} \bar{D}_{L 1} \Phi_{5} \ell_{R 1}+Y_{\ell 12}^{5} \bar{D}_{L 1} \Phi_{5} \ell_{R 2}+Y_{\ell 21}^{5} \bar{D}_{L 2} \Phi_{5} \ell_{R 1}+Y_{\ell 22}^{5} \bar{D}_{L 2} \Phi_{5} \ell_{R 2}+Y_{\ell 33}^{5} \bar{D}_{L 3} \Phi_{5} \ell_{R 3},
\end{aligned}
$$

which transforms under $Z_{2}$ as

$$
\begin{aligned}
\mathcal{L}_{M}^{Z_{6}} \stackrel{Z_{2}}{\rightarrow} & Y_{\chi 33}^{1} \chi_{1} \nu_{R 3}^{T} \mathcal{C}^{-1} \nu_{R 3} \\
& -Y_{\chi 11}^{2} \chi_{2} \nu_{R 2}^{T} \mathcal{C}^{-1} \nu_{R 2}+Y_{\chi 12}^{2} \chi_{2} \nu_{R 2}^{T} \mathcal{C}^{-1} \nu_{R 1}+Y_{\chi 12}^{2} \chi_{2} \nu_{R 1}^{T} \mathcal{C}^{-1} \nu_{R 2}-Y_{\chi 22}^{2} \chi_{2} \nu_{R 1}^{T} \mathcal{C}^{-1} \nu_{R 1} \\
& -Y_{\chi 11}^{3} \chi_{3} \nu_{R 2}^{T} \mathcal{C}^{-1} \nu_{R 2}+Y_{\chi 12}^{3} \chi_{3} \nu_{R 2}^{T} \mathcal{C}^{-1} \nu_{R 1}+Y_{\chi 12}^{3} \chi_{3} \nu_{R 1}^{T} \mathcal{C}^{-1} \nu_{R 2}-Y_{\chi 22}^{3} \chi_{3} \nu_{R 1}^{T} \mathcal{C}^{-1} \nu_{R 1} \\
& +Y_{D 11}^{1} \bar{D}_{L 1} \tilde{\Phi}_{2} \nu_{R 2}-Y_{D 12}^{1} \bar{D}_{L 2} \tilde{\Phi}_{1} \nu_{R 1}-Y_{D 21}^{1} \bar{D}_{L 1} \tilde{\Phi}_{1} \nu_{R 2}+Y_{D 22}^{1} \bar{D}_{L 1} \tilde{\Phi}_{1} \nu_{R 1}+Y_{D 33}^{1} \bar{D}_{L 3} \tilde{\Phi}_{1} \nu_{R 3} \\
& +i Y_{D 13}^{2} \bar{D}_{L 2} \tilde{\Phi}_{2} \nu_{R 3}-i Y_{D 23}^{2} \bar{D}_{L 1} \tilde{\Phi}_{2} \nu_{R 3}+i Y_{D 31}^{3} \bar{D}_{L 3} \tilde{\Phi}_{3} \nu_{R 2}-i Y_{D 32}^{3} \bar{D}_{L 3} \tilde{\Phi}_{3} \nu_{R 1} \\
& +Y_{\ell 11}^{4} \bar{D}_{L 2} \Phi_{5} \ell_{R 1}+Y_{\ell 12}^{4} \bar{D}_{L 2} \Phi_{5} \ell_{R 2}-Y_{\ell 21}^{4} \bar{D}_{L 1} \Phi_{5} \ell_{R 1}-Y_{\ell 22}^{4} \bar{D}_{L 1} \Phi_{5} \ell_{R 2}+i Y_{\ell 33}^{4} \bar{D}_{L 3} \Phi_{5} \ell_{R 3} \\
& -Y_{\ell 11}^{5} \bar{D}_{L 2} \Phi_{4} \ell_{R 1}-Y_{\ell 12}^{5} \bar{D}_{L 2} \Phi_{4} \ell_{R 2}+Y_{\ell 21}^{5} \bar{D}_{L 1} \Phi_{4} \ell_{R 1}+Y_{\ell 22}^{5} \bar{D}_{L 1} \Phi_{4} \ell_{R 2}-i Y_{\ell 33}^{5} \bar{D}_{L 3} \Phi_{4} \ell_{R 3}
\end{aligned}
$$

Thus, invariance under $Z_{6} \times Z_{2}$ implies the following constraints on the Yukawa couplings: 


$$
\begin{aligned}
& Y_{\chi 33}^{1}=Y_{\chi 33}^{1}, \quad Y_{\chi 12}^{2}=Y_{\chi 12}^{2}, \quad Y_{\chi 12}^{3}=Y_{\chi 12}^{3}, \quad Y_{\chi 11}^{2}=-Y_{\chi 22}^{2}, \quad Y_{\chi 11}^{3}=-Y_{\chi 22}^{3}, \\
& Y_{D 33}^{1}=Y_{D 33}^{1}, \quad Y_{D 11}^{1}=Y_{D 22}^{1}, \quad Y_{D 12}^{1}=-Y_{D 21}^{1}, \quad Y_{D 13}^{2}=-i Y_{D 23}^{2}, \quad Y_{D 31}^{3}=-i Y_{D 32}^{3}, \\
& Y_{\ell 11}^{4}=Y_{\ell 21}^{5}, \quad Y_{\ell 12}^{4}=Y_{\ell 22}^{5}, \quad Y_{\ell 21}^{4}=-Y_{\ell 11}^{5}, \quad Y_{\ell 22}^{4}=-Y_{\ell 12}^{5}, \quad Y_{\ell 33}^{4}=-i Y_{\ell 33}^{5},
\end{aligned}
$$

where all vanishing Yukawa couplings are omitted. In fact and as was done for the rotated symmetry (indirect realization), by brute force, one also could have used all the machinery encoded in the invariance equations, as given in Eqs. (60), (62), and (64), in order to obtain a system of linear equations involving Yukawa coupling constants. Solving this resulting system of linear equations would have provided us then with the symmetry constraints [Eq. (130)].

Thus, the $Z_{6} \times Z_{2}$ symmetry imposes some constraints on the Yukawa couplings that have to be taken into consideration when constructing mass terms after the relevant scalar fields acquire VEVs. The emergent $M_{R}$ and $M_{D}$ turn out to be

$$
M_{R}=\left(\begin{array}{ccc}
-Y_{\chi 22}^{2} v_{\chi_{2}}-Y_{\chi 22}^{3} v_{\chi_{3}} & Y_{\chi 12}^{2} v_{\chi_{2}}+Y_{\chi 12}^{3} v_{\chi_{3}} & 0 \\
- & Y_{\chi 22}^{2} v_{\chi_{2}}+Y_{\chi 22}^{3} v_{\chi_{3}} & 0 \\
- & - & Y_{\chi 33}^{1} v_{\chi_{1}}
\end{array}\right)
$$

and

$$
M_{D}=\left(\begin{array}{ccc}
Y_{D 22}^{1} v_{\Phi_{1}} & -Y_{D 21}^{1} v_{\Phi_{1}} & -i Y_{D 23}^{2} v_{\Phi_{2}} \\
Y_{D 21}^{1} v_{\Phi_{1}} & Y_{D 22}^{1} v_{\Phi_{1}} & Y_{D 23}^{2} v_{\Phi_{2}} \\
-i Y_{D 32}^{3} v_{\Phi_{3}} & Y_{D 32}^{3} v_{\Phi_{3}} & Y_{D 33}^{1} v_{\Phi_{1}}
\end{array}\right)
$$

One can check that the resulting $M_{\nu}$, through the seesaw mechanism, satisfies the texture $\mathbf{C}_{33}$.

As to $M_{\ell}$ we get

$$
M_{\ell}=\left(\begin{array}{ccc}
Y_{\ell 21}^{5} v_{\Phi_{4}}+Y_{\ell 11}^{5} v_{\Phi_{5}} & Y_{\ell 22}^{5} v_{\Phi_{4}}+Y_{\ell 12}^{5} v_{\Phi_{5}} & 0 \\
-Y_{\ell 11}^{5} v_{\Phi_{4}}+Y_{\ell 21}^{5} v_{\Phi_{5}} & -Y_{\ell 12}^{5} v_{\Phi_{4}}+Y_{\ell 22}^{5} v_{\Phi_{5}} & 0 \\
0 & 0 & -i Y_{\ell 33}^{5} v_{\Phi_{4}}+Y_{\ell 33}^{5} v_{\Phi_{5}}
\end{array}\right)
$$

Thus, and as an example, one can assume $v \approx v_{\Phi_{5}} \gg v_{\Phi_{4}}$ so as to get

$$
M_{\ell} \approx v\left(\begin{array}{ccc}
Y_{\ell 11}^{5} & Y_{\ell 12}^{5} & 0 \\
Y_{\ell 21}^{5} & Y_{\ell 22}^{5} & 0 \\
0 & 0 & Y_{\ell 33}^{5}
\end{array}\right)=v\left(\begin{array}{c}
\mathbf{a}^{T} \\
\mathbf{b}^{T} \\
\mathbf{c}^{T}
\end{array}\right)
$$

Another time, one can by just imposing some reasonable assumptions on the ratios of the "free" vectors diagonalize $M_{\ell} M_{\ell}^{\dagger}$ by an infinitesimal rotation, which puts us thus to a good approximation in the flavor basis, as desired.

\section{B. Type I seesaw direct realization of $\mathrm{C}_{11}, \mathrm{C}_{\mathbf{2 2}}$, and $\mathrm{C}_{\mathbf{1 3}}$}

Following the same method outlined in case $\mathbf{C}_{33}$, we state briefly the results of the cases $\mathbf{C}_{11}, \mathbf{C}_{22}$, and $\mathbf{C}_{13}$, in Tables XVIII, XIX, and XX, respectively.

\section{DIRECT REALIZATION OF TYPE II SEESAW WITH $Z_{2}^{\prime} \times Z_{2}$ SYMMETRY}

By the same token, we present now, within the type II seesaw scenario, a "direct"method which leads straight to the vanishing subtrace texture without relating it to zero textures by rotation. It is applicable again only for the four textures $\left(\mathbf{C}_{33}, \mathbf{C}_{11}, \mathbf{C}_{22}\right.$ and $\left.\mathbf{C}_{31}\right)$. Besides, the key idea behind this realization is having a permutation performed through the group factor $Z_{2}^{\prime}$.

\section{A. Type II seesaw direct realization of $\mathbf{C}_{\mathbf{3 3}}$}

Within the type II seesaw scenario, the term $Y_{i j}^{\nu a} H_{a}^{0} \nu_{L i}^{T} \mathcal{C}^{-1} \nu_{L j}$ in the Lagrangian of Eq. (107) is the term responsible for $M_{\nu}$ where we introduced three Higgs triplets. We introduce two Higgs doublets $\Phi_{b}$ responsible for $M_{\ell}$ through the term $Y_{i j}^{\ell a} \bar{D}_{L i} \Phi_{a} \ell_{R j}$ [see Eq. (109)]. We assume the field transformations defined in Table XXI. 
TABLE XVIII. The $Z_{6} \times Z_{2}$ symmetry seesaw type I realization of the vanishing subtrace $\mathbf{C}_{11}$. $\Phi_{a}$ are five SM Higgs doublets $(a=1 \ldots 5), D_{L}$ refers to the flavor three left-handed lepton doublets, while the three right-handed charged lepton singlets are denoted by $\ell_{R} . \omega$ denotes $e^{i \pi / 3}$.

Matter content and symmetry transformation (pattern $\mathbf{C}_{11}$ )

Symmetry under $Z_{6}$

$\nu_{R 1} \rightarrow \omega \nu_{R 1}$

$D_{L 1} \rightarrow \omega D_{L 1}$

$\nu_{R 2} \rightarrow \nu_{R 2}$
$D_{L 2} \rightarrow D_{L 2}$
$\Phi_{5} \rightarrow \omega^{3} \Phi_{5}$

$\nu_{R 3} \rightarrow \nu_{R 3}$

$\chi_{1} \rightarrow \omega^{4} \chi_{1}$

$\chi_{2} \rightarrow \chi_{2}$

$\chi_{3} \rightarrow \chi_{3}$

$\Phi_{4} \rightarrow \omega^{3} \Phi_{4}$

$D_{L 3} \rightarrow D_{L 3}$

$\Phi_{1} \rightarrow \Phi_{1}$

$\Phi_{2} \rightarrow \omega^{5} \Phi_{2}$

$\Phi_{3} \rightarrow \omega \Phi_{3}$

Symmetry under $Z_{2}$
$\nu_{R 1} \rightarrow \nu_{R 1}$
$\nu_{R 2} \rightarrow i \nu_{R 3}$
$D_{L 1} \rightarrow D_{L 1}$
$D_{L 2} \rightarrow i D_{L 3}$
$\nu_{R 3} \rightarrow-i \nu_{R 2}$
$D_{L 3} \rightarrow-i D_{L 2}$
$\ell_{R 1} \rightarrow \ell_{R 1}$
$\chi_{1} \rightarrow \chi_{1}$
$\Phi_{1} \rightarrow \Phi_{1}$
$\ell_{R 2} \rightarrow \ell_{R 2}$
$\chi_{2} \rightarrow \chi_{2}$
$\Phi_{2} \rightarrow \Phi_{2}$
$\ell_{R 3} \rightarrow \ell_{R 3}$

$\ell_{R 1} \rightarrow \omega^{4} \ell_{R 1}$

$\ell_{R 2} \rightarrow \omega^{3} \ell_{R 2}$

$\ell_{R 3} \rightarrow \omega^{3} \ell_{R 3}$ 
TABLE XXI. The $Z_{2}^{\prime} \times Z_{2}$ symmetry seesaw type II realization of the vanishing subtrace $\mathbf{C}_{33}$. $H$ are three triplet scalars, $D_{L}$ refers to the flavor three left-handed lepton doublets, while the three right-handed charged lepton singlets are denoted by $\ell_{R}$. The $\Phi$ denotes two SM Higgs doublets.

\begin{tabular}{lccc}
\hline \hline Matter content (pattern $\mathbf{C}_{33}$ ) & & & \\
\hline \multicolumn{1}{c}{$H$} & $D_{L}$ & $\ell_{R}$ & $\Phi$ \\
\hline Symmetry under $Z_{2}^{\prime}$ & & & \\
\hline$G_{H}^{\prime} H$ & $G_{D}^{\prime} D_{L}$ & $G_{\ell}^{\prime} \ell_{R}$ & $G_{\Phi}^{\prime} \Phi$ \\
$G_{H}^{\prime}=\operatorname{diag}(1,1,-1)$ & $G_{D}^{\prime}=\left(\begin{array}{ccc}0 & -i & 0 \\
+i & 0 & 0 \\
0 & 0 & 1\end{array}\right)$ & $G_{\ell}^{\prime}=\operatorname{diag}(1,1,1)$ & $G_{\Phi}^{\prime}=\left(\begin{array}{cc}0 & 1 \\
1 & 0\end{array}\right)$ \\
Symmetry under $Z_{2}$ & $G_{D} D_{L}$ & & $G_{\Phi} \Phi$ \\
$G_{H} H$ & $G_{D}=\operatorname{diag}(-1,-1,1)$ & $G_{\ell}=\operatorname{diag}(1,1,-1)$ & $G_{\Phi}=\operatorname{diag}(-1,-1)$ \\
$G_{H}=\operatorname{diag}(1,-1,-1)$ & &
\end{tabular}

By forming bilinear terms in order to see the transformations under $Z_{2}$, we get for $\nu_{L i}^{T} \nu_{L j}$,

$$
\nu_{L i}^{T} \nu_{L j} \stackrel{Z_{2}}{\cong}\left(\begin{array}{ccc}
1 & 1 & -1 \\
1 & 1 & -1 \\
-1 & -1 & 1
\end{array}\right),
$$

and so, when the bilinear $\nu_{L_{i}}^{T} \nu_{L_{j}}$ is combined with the transformation of $H_{a}$ under $Z_{2}$, we get

$$
\begin{aligned}
H_{1}^{0} \nu_{L i}^{T} \nu_{L j} \stackrel{Z_{2}}{\cong}\left(\begin{array}{ccc}
1 & 1 & -1 \\
1 & 1 & -1 \\
-1 & -1 & 1
\end{array}\right), \\
H_{2}^{0} \nu_{L i}^{T} \nu_{L j} \stackrel{Z_{2}}{\cong}\left(\begin{array}{ccc}
-1 & -1 & 1 \\
-1 & -1 & 1 \\
1 & 1 & -1
\end{array}\right), \\
H_{3}^{0} \nu_{L i}^{T} \nu_{L j} \stackrel{Z_{2}}{\cong}\left(\begin{array}{ccc}
-1 & -1 & 1 \\
-1 & -1 & 1 \\
1 & 1 & -1
\end{array}\right) .
\end{aligned}
$$

Thus, the Lagrangian terms in Eq. (107), responsible for $M_{\nu}$, which are due to the interaction with $H_{1}^{0}$ and are consistent with $Z_{2}$ symmetry are

$$
\begin{aligned}
L_{H_{1} \nu}^{Z_{2}} \propto & H_{1}^{0}\left(Y_{11}^{\nu 1} \mathcal{C}^{-1} \nu_{L 1}^{T} \nu_{L 1}+Y_{22}^{\nu 1} \nu_{L 2}^{T} \mathcal{C}^{-1} \nu_{L 2}+Y_{33}^{\nu 1} \nu_{L 3}^{T} \mathcal{C}^{-1} \nu_{L 3}\right. \\
& \left.+Y_{12}^{\nu 1} \nu_{L 1}^{T} \mathcal{C}^{-1} \nu_{L 2}+Y_{12}^{\nu 1} \nu_{L 2}^{T} \mathcal{C}^{-1} \nu_{L 1}\right)
\end{aligned}
$$

which transforms under $Z_{2}^{\prime}$ as

$$
\begin{aligned}
L_{H_{1} \nu}^{Z_{2}} & \stackrel{Z_{2}^{\prime}}{\rightarrow} H_{1}^{0}\left(-Y_{11}^{\nu 1} \nu_{L 2}^{T} \mathcal{C}^{-1} \nu_{L 2}-Y_{22}^{\nu 1} \nu_{L 1}^{T} \mathcal{C}^{-1} \nu_{L 1}+Y_{33}^{\nu 1} \nu_{L 3}^{T} \mathcal{C}^{-1} \nu_{L 3}\right. \\
& \left.+Y_{12}^{\nu 1} \nu_{L 1}^{T} \mathcal{C}^{-1} \nu_{L 2}+Y_{12}^{\nu 1} \nu_{L 2}^{T} \mathcal{C}^{-1} \nu_{L 1}\right) .
\end{aligned}
$$

Thus, invariance under $Z_{2} \times Z_{2}^{\prime}$ implies the constraint

$$
Y_{11}^{\nu 1}=-Y_{22}^{\nu 1}, \quad Y_{13}^{\nu 1}=Y_{23}^{\nu 1}=0 .
$$

By the same way, one can see the constraints on the Yukawa couplings due to interaction with $H_{2}^{0}$ and $H_{3}^{0}$, and we get

$$
\begin{array}{ll}
Y_{13}^{\nu 2}=i Y_{23}^{\nu 2}, & Y_{11}^{\nu 2}=Y_{22}^{\nu 2}=Y_{12}^{\nu 2}=0, \\
Y_{13}^{\nu 3}=-i Y_{23}^{\nu 3}, & Y_{11}^{\nu 3}=Y_{22}^{\nu 3}=Y_{12}^{\nu 3}=0 .
\end{array}
$$

So when $H_{a}^{0}$ gets a VEV $v_{a}^{H}$ we get $M_{\nu}$ in the form

$$
M_{\nu}=\left(\begin{array}{ccc}
-Y_{22}^{\nu 1} v_{1}^{H} & Y_{12}^{\nu 1} v_{1}^{H} & i\left(Y_{23}^{\nu 2} v_{2}^{H}-Y_{23}^{\nu 3} v_{3}^{H}\right) \\
- & Y_{22}^{\nu 1} v_{1}^{H} & \left(Y_{23}^{\nu 2} v_{2}^{H}+Y_{23}^{\nu 3} v_{3}^{H}\right) \\
- & - & Y_{33}^{\nu 1} v_{1}^{H}
\end{array}\right) .
$$

We see that the texture $\mathbf{C}_{33}$ is realized.

For the charged lepton mass matrix $M_{\ell}$, we follow the same procedure by forming bilinear terms in order to see the transformations under $Z_{2}$ :

$$
\begin{aligned}
\bar{D}_{L i} \ell_{R j} & \stackrel{Z_{2}}{\cong}\left(\begin{array}{ccc}
-1 & -1 & 1 \\
-1 & -1 & 1 \\
1 & 1 & -1
\end{array}\right) \Rightarrow \bar{D}_{L i} \ell_{R j} \Phi_{1} \stackrel{Z_{2}}{\cong} \bar{D}_{L i} \ell_{R j} \Phi_{2} \\
& \stackrel{Z_{2}}{\cong}\left(\begin{array}{ccc}
1 & 1 & -1 \\
1 & 1 & -1 \\
-1 & -1 & 1
\end{array}\right) .
\end{aligned}
$$

Thus, the Lagrangian terms in Eq. (109), responsible for $M_{\ell}$, which are due to the interaction with $\Phi_{1}, \Phi_{2}$ and are consistent with $Z_{2}$ symmetry are

$$
\begin{aligned}
L_{\Phi \ell}^{Z_{2}} \propto & \Phi_{1}\left(Y_{11}^{\ell 1} \bar{D}_{L 1} \ell_{R 1}+Y_{12}^{\ell 1} \bar{D}_{L 1} \ell_{R 2}+Y_{21}^{\ell 1} \bar{D}_{L 2} \ell_{R 1}\right. \\
& \left.+Y_{22}^{\ell 1} \bar{D}_{L 2} \ell_{R 2}+Y_{33}^{\ell 1} \bar{D}_{L 3} \ell_{R 3}\right) \\
& +\Phi_{2}\left(Y_{11}^{\ell 2} \bar{D}_{L 1} \ell_{R 1}+Y_{12}^{\ell 2} \bar{D}_{L 1} \ell_{R 2}+Y_{21}^{\ell 2} \bar{D}_{L 2} \ell_{R 1}\right. \\
& \left.+Y_{22}^{\ell 2} \bar{D}_{L 2} \ell_{R 2}+Y_{33}^{\ell 2} \bar{D}_{L 3} \ell_{R 3}\right)
\end{aligned}
$$

which transforms under $Z_{2}^{\prime}$ as 


$$
\begin{aligned}
L_{\Phi \ell}^{Z_{2}} \stackrel{Z_{2}^{\prime}}{\rightarrow} & \Phi_{2}\left(-i Y_{11}^{\ell 1} \bar{D}_{L 2} \ell_{R 1}-i Y_{12}^{\ell 1} \bar{D}_{L 2} \ell_{R 2}\right. \\
& \left.+i Y_{21}^{\ell 1} \bar{D}_{L 1} \ell_{R 1}+i Y_{22}^{\ell 1} \bar{D}_{L 1} \ell_{R 2}+Y_{33}^{\ell 1} \bar{D}_{L 3} \ell_{R 3}\right) \\
& +\Phi_{1}\left(-i Y_{11}^{\ell 2} \bar{D}_{L 2} \ell_{R 1}-i Y_{12}^{\ell 2} \bar{D}_{L 2} \ell_{R 2}+i Y_{21}^{\ell 2} \bar{D}_{L 1} \ell_{R 1}\right. \\
& \left.+i Y_{22}^{\ell 2} \bar{D}_{L 1} \ell_{R 2}+Y_{33}^{\ell 2} \bar{D}_{L 3} \ell_{R 3}\right) .
\end{aligned}
$$

Thus, invariance under $Z_{2} \times Z_{2}^{\prime}$ implies the constraint

$$
\begin{aligned}
Y_{11}^{\ell 1} & =i Y_{21}^{\ell 2}, \quad Y_{12}^{\ell 1}=i Y_{22}^{\ell 2}, \quad Y_{21}^{\ell 1}=-i Y_{11}^{\ell 2}, \\
Y_{22}^{\ell 1} & =-i Y_{12}^{\ell 2}, \quad Y_{33}^{\ell 1}=Y_{33}^{\ell 2}, \\
Y_{13}^{\ell 1(2)} & =Y_{23}^{\ell 1(2)}=Y_{31}^{\ell 1(2)}=Y_{32}^{\ell 1(2)}=0 .
\end{aligned}
$$

So when $\Phi_{a}^{0}$ gets a VEV $v_{a}^{\Phi}$, we get $M_{\ell}$ in the form

TABLE XXII. The $Z_{2}^{\prime} \times Z_{2}$ symmetry seesaw type II realization of the vanishing subtrace $\mathbf{C}_{11} . H$ are three triplet scalars, $D_{L}$ refers to the flavor three left-handed lepton doublets, while the three right-handed charged lepton singlets are denoted by $\ell_{R}$. The $\Phi$ denotes two SM Higgs doublets.

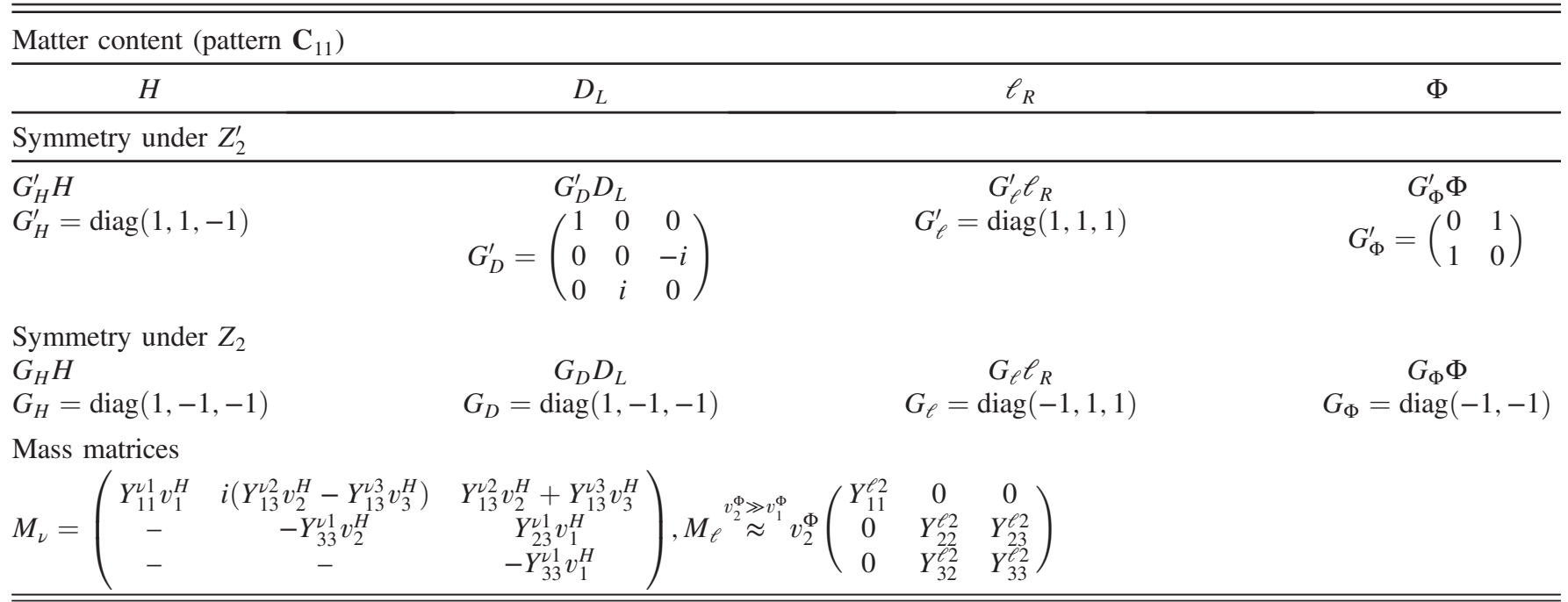

TABLE XXIII. The $Z_{2}^{\prime} \times Z_{2}$ symmetry seesaw type II realization of the vanishing subtrace $\mathbf{C}_{22}$. $H$ are three triplet scalars, $D_{L}$ refers to the flavor three left-handed lepton doublets, while the three right-handed charged lepton singlets are denoted by $\ell_{R}$. The $\Phi$ denotes two SM Higgs doublets.

Matter content (pattern $\mathbf{C}_{22}$ )

$\begin{array}{llll}H & D_{L} & \ell_{R} & \Phi\end{array}$

Symmetry under $Z_{2}^{\prime}$

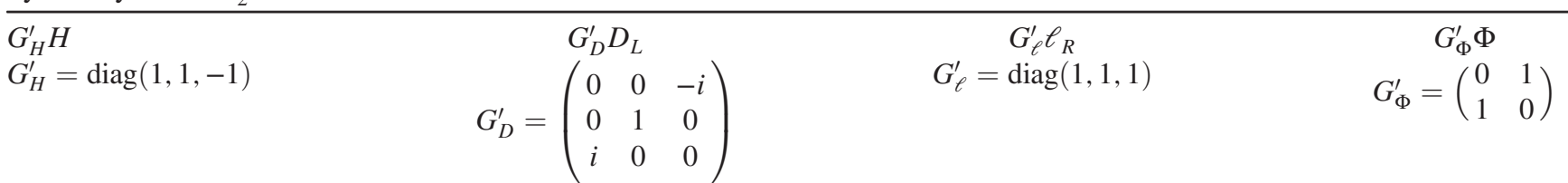

Symmetry under $Z_{2}$

$G_{H} H$

$G_{H}=\operatorname{diag}(1,-1,-1)$

Mass matrices

$M_{\nu}=\left(\begin{array}{ccc}-Y_{33}^{\nu 1} v_{1}^{H} & i\left(Y_{23}^{\nu 2} v_{2}^{H}-Y_{23}^{\nu 3} v_{3}^{H}\right) & Y_{13}^{\nu 1} v_{1}^{H} \\ - & Y_{22}^{\nu 1} v_{1}^{H} & \left(Y_{23}^{\nu 2} v_{2}^{H}+Y_{23}^{\nu 3} v_{3}^{H}\right) \\ - & - & Y_{33}^{\nu 1} v_{1}^{H}\end{array}\right), M_{\ell}{ }^{v_{2}^{\Phi} \gg v_{1}^{\Phi}} v_{2}^{\Phi}\left(\begin{array}{ccc}Y_{11}^{\ell 2} & 0 & Y_{13}^{\ell 2} \\ 0 & Y_{22}^{\ell 2} & 0 \\ -i Y_{31}^{\ell 2} & 0 & Y_{33}^{\ell 2}\end{array}\right)$ 
TABLE XXIV. The $Z_{2}^{\prime} \times Z_{2}$ symmetry seesaw type II realization of the vanishing subtrace $\mathbf{C}_{13} . H$ are three triplet scalars, $D_{L}$ refers to the flavor three left-handed lepton doublets, while the three right-handed charged lepton singlets are denoted by $\ell_{R}$. The $\Phi$ denotes two SM Higgs doublets.

Matter content (pattern $\mathbf{C}_{13}$ )

\begin{tabular}{|c|c|c|c|c|}
\hline$H$ & & $D_{L}$ & $\ell_{R}$ & $\Phi$ \\
\hline \multicolumn{5}{|l|}{ Symmetry under $Z_{2}^{\prime}$} \\
\hline $\begin{array}{l}G_{H}^{\prime} H \\
G_{H}^{\prime}=\operatorname{diag}(1,-1,-1)\end{array}$ & 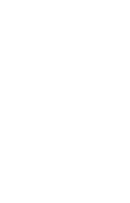 & $\begin{array}{c}G_{D}^{\prime} D_{L} \\
G_{D}^{\prime}=\left(\begin{array}{ccc}0 & 0 & 1 \\
0 & 1 & 0 \\
1 & 0 & 0\end{array}\right)\end{array}$ & $\begin{aligned} G_{\ell}^{\prime} \ell_{R} \\
G_{\ell}^{\prime}=\operatorname{diag}(1,1,1)\end{aligned}$ & $\begin{array}{c}G_{\Phi}^{\prime} \Phi \\
G_{\Phi}^{\prime}=\left(\begin{array}{ll}0 & 1 \\
1 & 0\end{array}\right)\end{array}$ \\
\hline $\begin{array}{l}\text { Symmetry under } Z_{2} \\
G_{H} H \\
G_{H}=\operatorname{diag}(1,1,-1)\end{array}$ & & $\begin{array}{c}G_{D} D_{L} \\
G_{D}=\operatorname{diag}(-1,1,-1)\end{array}$ & $\begin{array}{c}G_{\ell} \ell_{R} \\
G_{\ell}=\operatorname{diag}(1,-1,1)\end{array}$ & $\begin{array}{c}G_{\Phi} \Phi \\
G_{\Phi}=\operatorname{diag}(-1,-1)\end{array}$ \\
\hline $\begin{array}{l}\text { Mass matrices } \\
M_{\nu}=\left(\begin{array}{c}Y_{33}^{\nu 1} v_{1}^{H}-Y_{33}^{\nu 2} v_{2}^{H} \\
- \\
-\end{array}\right.\end{array}$ & $\begin{array}{c}-Y_{32}^{\nu 3} v_{3}^{H} \\
Y_{22}^{\nu 1} v_{1}^{H} \\
-\end{array}$ & $\left.\begin{array}{c}Y_{13}^{\nu 1} v_{1}^{H} \\
Y_{23}^{\nu 3} v_{3}^{H} \\
Y_{33}^{\nu 1} v_{1}^{H}+Y_{33}^{\nu 2} v_{2}^{H}\end{array}\right), M_{\ell} \stackrel{v_{2}^{\Phi} \gg v_{1}^{\Phi}}{\approx} v_{2}^{\Phi}$ & $\left(\begin{array}{ccc}Y_{11}^{\ell 2} & 0 & Y_{13}^{\ell 2} \\
0 & Y_{22}^{\ell 2} & 0 \\
Y_{31}^{\ell 2} & 0 & Y_{33}^{\ell 2}\end{array}\right)$ & \\
\hline
\end{tabular}

$M_{\ell}=\left(\begin{array}{ccc}Y_{11}^{\ell 1} v_{1}^{\Phi}+i Y_{21}^{\ell 1} v_{2}^{\Phi} & Y_{12}^{\ell 1} v_{1}^{\Phi}+i Y_{22}^{\ell 1} v_{2}^{\Phi} & 0 \\ Y_{21}^{\ell 1} v_{1}^{\Phi}-i Y_{11}^{\ell 1} v_{2}^{\Phi} & Y_{22}^{\ell 1} v_{1}^{\Phi}-i Y_{12}^{\ell 1} v_{2}^{\Phi} & 0 \\ 0 & 0 & Y_{33}^{\ell 1}\left(v_{1}^{\Phi}+v_{2}^{\Phi}\right)\end{array}\right)$

Thus, when $v \approx v_{2}^{\Phi} \gg v_{1}^{\Phi}$, we get

$$
M_{\ell}=v\left(\begin{array}{ccc}
i Y_{21}^{\ell 1} & i Y_{22}^{\ell 1} & 0 \\
-i Y_{11}^{\ell 1} & -i Y_{12}^{\ell 1} & 0 \\
0 & 0 & Y_{33}^{\ell 1}
\end{array}\right)=v\left(\begin{array}{c}
\mathbf{a}^{T} \\
\mathbf{b}^{T} \\
\mathbf{c}^{T}
\end{array}\right)
$$

One can by just imposing some reasonable assumptions on the ratios of the free vectors diagonalize $M_{\ell} M_{\ell}^{\dagger}$ by an infinitesimal rotation, which puts us thus to a good approximation in the flavor basis, as desired.

\section{B. Type II seesaw direct realization of $\mathrm{C}_{11}, \mathrm{C}_{22}$, and $\mathrm{C}_{\mathbf{1 3}}$}

Following the same method outlined in case $\mathbf{C}_{33}$, we state briefly the results of the cases $\mathbf{C}_{11}, \mathbf{C}_{22}$, and $\mathbf{C}_{13}$, in Tables XXII, XXIII, and XXIV, respectively.

\section{SUMMARY, DISCUSSION, AND CONCLUSION}

We have studied a specific texture characterized by one vanishing subtrace of the neutrino mass matrix. We found that all textures, whether they be of inverted or normal type, can accommodate the recent experimental bounds. Moreover, four textures of inverted type can accommodate data in case one neutrino mass is zero. We have carried out a complete phenomenological and analytic analysis, but did not state the analytic expressions, as they are too cumbersome, even the first terms in a Taylor expansion in powers of $s_{13}$. Finally, for the model building of the texture, we first proposed a generic strategy to justify such a specific texture form based on finding a corresponding symmetry implying certain zeros at $M_{\nu 0}$, which when rotated to a new rotated symmetry leads to the desired form of vanishing subtrace in $M_{\nu}$. We applied this strategy for both types of seesaw scenarios and in both invertible and singular neutrino mass matrices. We also presented a direct method to realize the textures without rotation for both types of seesaw scenarios based on discrete symmetry.

In all these theoretical models, the spontaneous symmetry breaking of a discrete symmetry $Z_{n} \times Z_{m}$, triggered by some fields $(\Phi, \chi)$ —some of which are very heavytaking a VEV, led by construction to a texture of vanishing subtrace, and this presumably happens at high scale. However, the question arises as to whether the running of the Yukawa couplings from high scale to weak scale spoils the form of the texture. In our work we assumed this change is slight, and that the texture would be kept when running to weak scale, in line with [14]. However, it was argued in [10] that the "entries-equality" condition is not stable against radiative corrections, and surely this question is worthy of a thorough analysis in its own right.

Moreover, we have not discussed the scalar and Higgs potential. In Appendix we stated the general form of the renormalizable scalar-Higgs potential in one case $\left(C_{33}\right)$ respecting the discrete symmetry $\left(Z_{2} \times Z_{6}\right)$, and therein we put in constraints on the coupling constants and stated the tadpole equations and the corresponding mass matrices. 
The questions of stability and perturbative unitarity, in that the potential is copositive and bounded from below or at least it accepts local minima with sufficiently large decaying timescales through tunneling, and that the coupling constants are small enough for perturbative expansion, are all assumed to be met through tuning of the potential parameters. Surely, a term such as $\Phi^{\dagger} \Phi \chi^{\dagger} \chi$ would break the discrete symmetry when $\chi$ takes a VEV, and here we wonder whether the $\Phi$ can behave as an SM Higgs field, as we assumed in our realization models, in which case we ask how its mass is kept small, whereas such a term gives a contribution of the form $Y v_{\chi}^{2}$ where $v_{\chi}$ is expected to be high. Again, we assume a sort of fine-tuning imposing the corresponding $Y$ to be too small, and this is reminiscent of the Higgs hierarchy problem, where even in the SM and at tree levels, one needs fine-tuning in order to keep the Higgs mass at electroweak scale assuming it is coupled to heavy scalars.

Finally, the existence in our realization models of many electroweak doublets $\Phi$ 's which get a VEV at the electroweak scale could lead to an interesting phenomenology of the extra states at the Large Hadron Collider (LHC). We did not discuss this, but rather assumed again a fine-tuning position. First, the parameters of the Higgsscalar potential are assumed to be fine-tuned so that several Higgs fields $\Phi$ get a VEV $v_{\Phi}$ at the electroweak scale, while other fields $\chi$ get a VEV $v_{\chi}$ at high scale. Second, the corresponding "quartic" Yukawas for some of these electroweak doublets $\left(Y_{\Phi} \Phi^{4}\right)$ are tuned to be high, so that the "low scale" contribution to their masses $\left(Y_{\Phi} v_{\Phi}^{2}\right)$, when added to the "high scale" contribution to their masses $\left(Y_{\Phi \chi} v_{\chi}^{2}\right)$ originating from the coupling term $\left(Y_{\Phi \chi} \Phi^{2} \chi^{2}\right)$, is in such a way that the resulting mass for all the electroweak doublets $\Phi$ 's, except the SM one, are beyond the reach of current accelerators. Actually, this is a "common" assumption because fine-tuning of parameters is required whenever there are two different scales in the theory, which are generated by the Higgs VEVs [21]. In [18], a similar enriched scalar sector with three Higgs doublets and two scalar gauge singlets was studied, and again a high level of fine-tuning was required in order to eliminate the large radiative corrections, originated from the existence of a high scale in the model, through renormalization to the mass of light scalars.

We hope to address some of the above mentioned points in the future.

\section{ACKNOWLEDGMENTS}

M. A. thanks the staff members of the CPPMMarseille and LPTM-Cergy-Pontoise Laboratories. N. C. acknowledges support from ICTP-Associate program, Institute of Theoretical Physics - Chinese Academy of Sciences - President's International Fellowship Initiative (ITP-CAS-PIFI) program, and Humboldt Foundation.

\section{APPENDIX: SCALAR POTENTIAL FOR THE CASE $C_{33}$ UNDER $Z_{2} \times Z_{6}$ SYMMETRY}

Looking at Table XVII as a representative case of direct realization, ${ }^{3}$ we see that for the sake of constructing the scalar potential, we can drop the two gauge singlets so that we have five doublets, $\phi=\left(\phi_{1}, \phi_{2}, \phi_{3}, \phi_{4}, \phi_{5}\right)^{T}$, and one singlet $\chi$. The scalar fields transform under $Z_{2} \times Z_{6}$ as follows $\left(\omega=e^{\pi i / 3}\right)$ :

$$
\begin{aligned}
& \phi \stackrel{Z_{6}}{\rightarrow} \operatorname{diag}\left(1, \omega, \omega^{5}, \omega^{3}, \omega^{3}\right) \phi=\mathbf{U}_{6} \phi, \\
& \phi \stackrel{Z_{2}}{\rightarrow}\left(\begin{array}{ccccc}
1 & 0 & 0 & 0 & 0 \\
0 & -1 & 0 & 0 & 0 \\
0 & 0 & 1 & 0 & 0 \\
0 & 0 & 0 & 0 & i \\
0 & 0 & 0 & -i & 0
\end{array}\right) \phi=\mathbf{U}_{2} \phi, \\
& \chi \stackrel{Z_{6}}{\rightarrow} \omega^{4} \chi, \quad \chi \stackrel{Z_{2}}{\rightarrow} \chi .
\end{aligned}
$$

Dictated by gauge symmetry, we see directly that the most general renormalizable potential expressing the coupling amidst the $\phi$ and $\chi$ fields can be restricted to the following form:

$$
\begin{aligned}
V & =V_{\phi}+V_{\chi}+V_{\phi \chi}, \\
V_{\phi} & =\mu_{a b} \phi_{a}^{\dagger} \phi_{b}+\lambda_{a b c d} \phi_{a}^{\dagger} \phi_{b} \phi_{c}^{\dagger} \phi_{d}, \\
V_{\chi} & =m_{\chi}^{2} \chi^{\dagger} \chi+\lambda_{\chi}\left(\chi^{\dagger} \chi\right)^{2}, \\
V_{\phi \chi} & =\lambda_{\chi a b}\left(\chi^{\dagger} \chi\right)\left(\phi_{a}^{\dagger} \phi_{b}\right),
\end{aligned}
$$

where $\mu_{a b}$ and $m_{\chi}^{2}$ are mass square parameters while $\lambda_{a b c d}$, $\lambda_{\chi a b}$, and $\lambda_{\chi}$ are Yukawa coupling coefficients of fields having the corresponding indices. The sum convention over repeated indices is implied. We see directly from the definition of quartic couplings that $\lambda_{a b c d}=\lambda_{c d a b}$. Together with Hermiticity, the coupling coefficients satisfy

$$
\begin{aligned}
\mu_{a b} & =\mu_{b a}^{*}, \quad \lambda_{\chi a b}=\lambda_{\chi b a}^{*}\left(m_{\chi}^{2} \text { and } \lambda_{\chi} \text { are real }\right), \\
\lambda_{a b c d} & =\lambda_{c d a b}=\lambda_{b a d c}^{*} .
\end{aligned}
$$

In constructing the scalar potential we follow closely the notation of $[22,23]$.

Let us find the independent couplings from the constraints of Eq. (A3) alone. Since this is a common problem, we consider $n$ doublets $\phi_{a}$ (in our case $n=5$ ) with one gauge singlet scalar $\chi$. In order to ease the counting and also to provide notational simplicity, we arrange the indices of the mass parameters and Yukawa couplings in 2-tuples and 4-tuples as $(a, b)$ and $(a, b, c, d)$. Thus, it is easy to count the number of independent couplings for each of the quadratic $\left(\mu_{a b}\right)$ and cubic $\left(\lambda_{\chi a b}\right)$ couplings as

\footnotetext{
${ }^{3}$ We could equally choose the case $C_{33}$ of indirect realization as presented in Table $\mathrm{V}$ but we chose the case of direct realization since it is simpler.
} 


$$
(a, b): a \leq b, \quad \text { counting } 1+2+3+\cdots+n=\sum_{k=1}^{n} k=\frac{n(n+1)}{2} .
$$

For the quartic coefficients, one can, using the constraints of Eq. (A3), span all the couplings once and only once, by restricting to

$$
\begin{aligned}
& (a, b, c, d):\{a=\operatorname{Min}\{a, b, c, d\} \wedge a \notin\{b, c, d\}\} \text { counting } \sum_{k=1}^{n}(n-k)^{3}=\frac{n^{2}(n-1)^{2}}{4} \\
& (a, a, c, d):\{c \leq d, a=\operatorname{Min}\{a, c, d\} \wedge a \notin\{c, d\}\} \\
& (a, b, a, d):\{b \leq d, a=\operatorname{Min}\{a, b, d\} \wedge a \notin\{b, d\}\} \text { counting } 3 \sum_{k=1}^{n} \sum_{j=k+1}^{n}(n-j+1)=\frac{n\left(n^{2}-1\right)}{2} \\
& (a, b, c, a):\{b \leq c, a=\operatorname{Min}\{a, b, c\} \wedge a \notin\{b, c\}\} \\
& (a, a, a, d):\{a<d\} \text { counting } \sum_{k=1}^{n}(n-k)=\frac{n(n-1)}{2}, \\
& (a, a, a, a) \text { counting } n .
\end{aligned}
$$

By adding the number of independent parameters, we get $\frac{n^{4}+3 n^{2}}{4}$, giving 175 independent quartic Yukawa coefficients. Imposing the invariance under $Z_{2} \times Z_{6}$ symmetry we find the following constraints:

$$
\left.\begin{array}{l}
\mu_{c d}=\mathbf{U}_{\alpha a c}^{*} \mathbf{U}_{\alpha b d} \mu_{a b} \\
\lambda_{x c d}=\mathbf{U}_{\alpha a c}^{*} \mathbf{U}_{\alpha b d} \lambda_{x a b} \\
\lambda_{e f g h}=\mathbf{U}_{\alpha a e}^{*} \mathbf{U}_{\alpha b f} \mathbf{U}_{\alpha c g}^{*} \mathbf{U}_{\alpha d h} \lambda_{a b c d}
\end{array}\right\} \alpha \in\{\mathbf{2 , 6}\} .
$$

We end up with the following independent Yukawa couplings (5 quadratic, 5 cubic, and 33 quartic coefficients) shown in Table XXV.

We assume that the Yukawa coupling are such that the potential gets local minima, around one of which the fields are expanded as follows:

$$
\phi_{k}=\left(\begin{array}{c}
\varphi_{k}^{+} \\
\frac{1}{\sqrt{2}}\left(v_{k}+\varphi_{k}^{o}\right)
\end{array}\right), \quad \chi=\frac{v_{\chi}+\chi^{o}}{\sqrt{2}} .
$$

In terms of the fields $\left(\varphi_{a}^{+}, \varphi_{b}^{o}, \chi^{o}\right)$, the potential can be decomposed into constant, linear, quadratic, cubic, and quartic terms. Being in a local minimum means that the linear term is vanishing, whence we get the tadpole conditions:

$$
\begin{gathered}
{\left[\mu_{a b}+\lambda_{a b c d} v_{c}^{*} v_{d}\right] v_{b}+\frac{1}{2} \lambda_{\chi a b} v_{\chi}^{*} v_{\chi} v_{b}=0,} \\
\left(m_{\chi}^{2}+\frac{1}{2} \lambda_{\chi a b} v_{a}^{*} v_{b}\right) v_{\chi}=0
\end{gathered}
$$

As to the quadratic term, it gives the mass matrices

$$
V_{2}=\varphi_{a}^{-}\left(\mathbf{M}_{ \pm}^{2}\right)_{a b} \varphi_{b}^{+}+\frac{1}{2} \mathcal{N}^{T} \mathbf{M}_{o}^{2} \mathcal{N}
$$

where $\mathcal{N}$ represents the neutral fields organized as

$$
\begin{gathered}
\mathcal{N} \equiv\left(\operatorname{Re}\left[\varphi_{1}^{o}\right], \ldots, \operatorname{Re}\left[\varphi_{5}^{o}\right], \operatorname{Im}\left[\varphi_{1}^{o}\right], \ldots\right. \\
\left.\operatorname{Im}\left[\varphi_{5}^{o}\right], \operatorname{Re}\left[\chi^{o}\right], \operatorname{Im}\left[\chi^{o}\right]\right)^{T}
\end{gathered}
$$

The Hermitian $5 \times 5$ matrix $\mathbf{M}_{ \pm}^{2}$, in Eq. (A10), represents the mass matrix of charged fields and is given as

TABLE XXV. Independent Yukawa coupling in the case $C_{33}$ under $Z_{2} \times Z_{6}$ symmetry.

\begin{tabular}{lc}
\hline \hline Independent Yukawa couplings & $\in$ which number field \\
\hline$\mu_{11}, \mu_{22}, \mu_{33}, \mu_{44}=\mu_{5,5} ; \lambda_{\chi 11}, \lambda_{\chi 22}, \lambda_{\chi 33}, \lambda_{\chi 44}=\lambda_{\chi 5,5}$ & $\mathbb{R}$ \\
$\mu_{45} ; \lambda_{\chi 45}$ & $i \mathbb{R}$ \\
$\lambda_{1111}, \lambda_{1122}, \lambda_{1133}, \lambda_{1144}=\lambda_{1155}, \lambda_{1221}, \lambda_{1331}, \lambda_{1441}=\lambda_{1551}$ & $\mathbb{R}$ \\
$\lambda_{2222}, \lambda_{3333}, \lambda_{4444}=\lambda_{5555}, \lambda_{2233}, \lambda_{3344}=\lambda_{3355}, \lambda_{2244}=\lambda_{2255}$ & \\
$\lambda_{2332}, \lambda_{3443}=\lambda_{3553}, \lambda_{2442}=\lambda_{2552}, \lambda_{4455}, \lambda_{4545}, \lambda_{4554}$ & \\
$\lambda_{1145}, \lambda_{1451}, \lambda_{2245}, \lambda_{2452}, \lambda_{3345}, \lambda_{3453}$ & $i \mathbb{R}$ \\
$\lambda_{1213}, \lambda_{1415}, \lambda_{1414}=-\lambda_{1515}, \lambda_{2324}=-i \lambda_{2325}, \lambda_{2343}=-i \lambda_{2353}$ & $\mathbb{C}$ \\
$\lambda_{2434}=\lambda_{2535}, \lambda_{2435}=\lambda_{2534}, \lambda_{4445}=-\lambda_{4555}^{*}$ & $\mathbb{C}$ \\
\hline \hline
\end{tabular}




$$
\left(\mathbf{M}_{ \pm}^{2}\right)_{a b}=\mu_{a b}+\lambda_{a b c d} v_{c}^{*} v_{d}+\frac{1}{2} \lambda_{\chi a b} v_{\chi}^{*} v_{\chi}
$$

while the $12 \times 12$ matrix $\mathbf{M}_{o}^{2}$ for neutral fields is expressed in terms of block matrices as

$$
\mathbf{M}_{o}^{2}=\left(\begin{array}{cc}
\mathbf{M}_{\varphi}^{\mathbf{2}} & \mathbf{M}_{\chi \varphi}^{\mathbf{2}} \\
\mathbf{M}_{\chi \varphi}^{\mathbf{2} T} & \mathbf{M}_{\chi}^{\mathbf{2}}
\end{array}\right)
$$

where the $10 \times 10$ matrix $\mathbf{M}_{\varphi}^{2}$ is in turn given in terms of block matrices as

$$
\mathbf{M}_{\varphi}^{2}=\left(\begin{array}{cc}
\mathbf{M}_{\mathbf{R}}^{2} & \mathbf{M}_{\mathbf{R} \mathbf{I}}^{2} \\
\mathbf{M}_{\mathbf{R} \mathbf{I}}^{2} & \mathbf{M}_{\mathbf{I}}^{2}
\end{array}\right) \quad \text { with } \begin{aligned}
& \left(\mathbf{M}_{\mathbf{R}}^{2}\right)_{a b}=\operatorname{Re}\left[\left(\mathbf{M}_{ \pm}^{2}\right)_{a b}+\lambda_{a c d b} v_{c} v_{d}^{*}+\lambda_{a c b d} v_{c} v_{d}\right] \\
& \left(\mathbf{M}_{\mathbf{I}}^{2}\right)_{a b}=\operatorname{Re}\left[\left(\mathbf{M}_{ \pm}^{2}\right)_{a b}+\lambda_{a c d b} v_{c} v_{d}^{*}-\lambda_{a c b d} v_{c} v_{d}\right], \\
& \left(\mathbf{M}_{\mathbf{R} \mathbf{I}}^{2}\right)_{a b}=-\operatorname{Im}\left[\left(\mathbf{M}_{ \pm}^{2}\right)_{a b}+\lambda_{a c d b} v_{c} v_{d}^{*}-\lambda_{a c b d} v_{c} v_{d}\right]
\end{aligned}
$$

and the $10 \times 2$ matrix $\mathbf{M}_{\chi \varphi}^{2}$ is given in terms of four $5 \times 1$ matrices as follows:

$$
\mathbf{M}_{\chi \varphi}^{2}=\left(\begin{array}{cc}
\mathbf{M}_{\chi \varphi \mathbf{R}}^{2} & \mathbf{M}_{\chi \varphi \mathbf{R} \mathbf{I}}^{2} \\
\mathbf{M}_{\chi \varphi \mathbf{I} \mathbf{R}}^{2} & \mathbf{M}_{\chi \varphi \mathbf{I}}^{2}
\end{array}\right) \text { with } \begin{aligned}
& \left(\mathbf{M}_{\chi \varphi \mathbf{R}}^{2}\right)_{a 1}=\operatorname{Re}\left[\lambda_{\chi a b}\left(v_{\chi}^{*} v_{b}+v_{\chi} v_{b}\right)\right], \\
& \left.\left(\mathbf{M}_{\chi \varphi \mathbf{I} \mathbf{I}}^{2}\right)_{a 1}\right)_{a 1}=\operatorname{Re}\left[\lambda_{\chi a b}\left(v_{\chi}^{*} v_{b}-v_{\chi} v_{b}\right)\right], \\
& \left.\left(\mathbf{M}_{\chi \varphi \mathrm{I} \mathbf{R}}^{2}\right)_{a 1}=-\operatorname{Im}\left[v_{\chi}^{*} v_{b}+v_{\chi} v_{b}\right)\right],
\end{aligned}
$$

Finally, the $2 \times 2$ matrix $\mathbf{M}_{\chi}^{2}$ is given by

$$
\mathbf{M}_{\chi}^{2}=\left(m_{\chi}^{2}+\frac{1}{2} \lambda_{\chi a b} v_{a}^{*} v_{b}\right) \operatorname{diag}(1,1)
$$

Note that $\mathbf{M}_{ \pm}^{2}$ is Hermitian, whereas $\mathbf{M}_{\mathbf{R}}^{2}, \mathbf{M}_{\mathbf{I}}^{2}, \mathbf{M}_{\chi}^{2}$ are real symmetric. The matrices $\mathbf{M}_{\mathbf{R} \mathbf{I}}^{2}, \mathbf{M}_{\chi \varphi \mathbf{R}}^{2}, \mathbf{M}_{\chi \varphi \mathbf{I}}^{2}, \mathbf{M}_{\chi \varphi \mathbf{R} \mathbf{I}}^{2}$, $\mathbf{M}_{\chi \varphi \mathbf{I R}}^{2}$ are real, so we get $\mathbf{M}_{o}^{2}$ as real symmetric.
One should diagonalize the mass matrices in order to get the physical masses, but we anticipate at least three vanishing masses which would correspond to the would be Goldstone bosons. With such a large number of free parameters, we do not carry out this task, but just assume that the Yukawa couplings are chosen in their parameters' space so that the mass spectrum is such that one doublet would play the role of the SM Higgs, whereas the others would be outside the LHC reach.
[1] See e.g., K. Nakamura et al. (Particle Data Group), J. Phys. G 37, 075021 (2010).

[2] See e.g., Y. Fukuda et al., Phys. Lett. B 436, 33 (1998); Phys. Rev. Lett. 81, 1562 (1998); C. K. Jung, C. McGrew, T. Kajita, and T. Mann, Annu. Rev. Nucl. Part. Sci. 51, 451 (2001), and references therein.

[3] P. H. Frampton, S. L. Glashow, and D. Marfatia, Phys. Lett. B 536, 79 (2002); Z. Z. Xing, Phys. Lett. B 530, 159 (2002); H. Fritzsch, Z. Z. Xing, and S. Zhou, J. High Energy Phys. 09 (2011) 083.

[4] A. Merle and W. Rodejohann, Phys. Rev. D 73, 073012 (2006).

[5] E. I. Lashin and N. Chamoun, Phys. Rev. D 85, 113011 (2012).

[6] L. Lavoura, Phys. Lett. B 609, 317 (2005); E. I. Lashin and N. Chamoun, Phys. Rev. D 78, 073002 (2008); 80, 093004 (2009).

[7] E. I. Lashin, N. Chamoun, C. Hamzaoui, and S. Nasri, Phys. Rev. D 89, 093004 (2014); 91, 113014 (2015); 96, 015003 (2017).
[8] H. A. Alhendi, E. I. Lashin, and A. A. Mudlej, Phys. Rev. D 77, 013009 (2008).

[9] T. Kitabayashi and M. Yasue, Phys. Lett. B 621, 133 (2005); W. Grimus, S. Kaneko, L. Lavoura, H. Sawanaka, and M. Tanimoto, J. High Energy Phys. 01 (2006) 110.

[10] Ji-Yuan Liu and Shun Zhou, Phys. Rev. D 87, 093010 (2013).

[11] S. Dev, R. R. Gautam, and L. Singh, Phys. Rev. D 87, 073011 (2013).

[12] F. Capozzi, E. D. Valentino, E. Lisi, A. Marrone, A. Melchiorri, and A. Palazzo, Phys. Rev. D 95, 096014 (2017).

[13] F. P. An et al. (DAYA-BAY Collaboration), Phys. Rev. Lett. 108, 171803 (2012).

[14] W. Grimus and L. Lavoura, Eur. Phys. J. C 39, 219 (2005); A. Dighe, S. Goswami, and P. Roy, Phys. Rev. D 76, 096005 (2007); S. Luo and Z.-z. Xing, Phys. Rev. D 86, 073003 (2012).

[15] Heinrich Päs and W. Rodejohann, New J. Phys. 17, 115010 (2015). 
[16] E. Andreotti et al., Astropart. Phys. 34, 822 (2011).

[17] M. Bargiotti et al., Riv. Nuovo Cimento 23N3, 1 (2000), arXiv:hep-ph/0001293.

[18] W. Grimus and L. Lavoura, Phys. Lett. B 572, 189 (2003).

[19] E. Ma, Phys. Lett. B 583, 157 (2004).

[20] T. P. Cheng and L.F. Li, Phys. Rev. D 22, 2860 (1980); R. N. Mohapatra and G. Senjanovic, Phys. Rev. D 23, 165 (1981).
[21] Particle and Astroparticle Physics, Series in High Energy Physics, Cosmology, and Gravitation edited by U. Sarkar (Taylor and Francis Group, London, 2008).

[22] F. J. Botella and J. P. Silva, Phys. Rev. D 51, 3870 (1995).

[23] G. C. Branco, L. Lavoura, and J. P. Silva, CP Violation (Oxford University Press, Oxford U.K., 1999). 